\title{
High Speed Wavelength Tuning of SGDBR Lasers for Optical Coherence Tomography Applications
}

\author{
A Thesis Presented to the \\ Electrical Engineering Department Faculty of \\ California Polytechnic State University, San Luis Obispo
}

In Partial Fulfillment

Of the Requirements for the

Master of Science Degree in Electrical Engineering

by

Benjamin James Maher

December 2008 
(C) 2009

Benjamin James Maher

ALL RIGHTS RESERVED 


\section{COMMITTEE MEMBERSHIP}

TITLE: $\quad$ High Speed Wavelength Tuning of SGDBR Lasers for Optical Coherence Tomography Applications
AUTHOR:
Benjamin James Maher
DATE SUBMITTED:
January 2009
COMMITTEE CHAIR:
Dennis Derickson, Assistant Professor
COMMITTEE MEMBER: $\quad$ Xiaomin Jin, Assistant Professor
COMMITTEE MEMBER: John Saghri, Associate Professor 


\title{
ABSTRACT \\ High Speed Wavelength Tuning of SGDBR Lasers for Optical
}

\section{Coherence Tomography Applications}

\author{
Benjamin James Maher
}

The application of Sample Grated Distributed Bragg Reflector (SGDBR) wavelength tunable lasers for swept-wavelength Optical Coherence Tomography (OCT) is explored. OCT is a method of measuring reflectivity versus distance into samples under test with a focused infrared light source. Swept wavelength OCT requires a laser light source that is capable of sweeping its wavelength quickly over the entire wavelength range of the tunable laser. Fast sweeping of the laser's wavelength enables real-time imaging of a wide surface area of the surface under test. This thesis will show that SGDBR lasers can be designed to meet the fast wavelength ramp speeds of swept wavelength OCT and to even exceed the capability of present swept-wavelength OCT source solutions.

SGDBR lasers were originally developed for telecommunications applications using Wavelength Division Multiplexing. In the telecommunications application, the wide wavelength tuning range of the device $(1520-1575 \mathrm{~nm})$ is utilized but the devices are only required to change wavelength over $50 \mathrm{~ms}$ time intervals. Research on SGDBR lasers has shown that wavelength switching speeds of $5 \mathrm{~ns}$ have been obtained using predistortion of the current drive waveforms [9]. This thesis explores the inherent modulation speed of chip-level and packaged SGDBR lasers and the associated capability to make high speed continuous wavelength ramps for swept wavelength OCT. It will be shown that frequency modulation speeds of over $100 \mathrm{MHz}$ can be accomplished with the laser drive and packaging techniques presented in this work. The result of the work shows that SGDBR lasers are very promising sources for swept wavelength OCT applications.

In order to understand the present generation OCT application in more detail, work is first presented demonstrating the capability of white-light interferometry OCT in a meat tenderness measurement application. White light interferometer measurement OCT has been the standard solution for OCT measurements for at least 15 years. Measurement of a range of beef samples was done in conjunction with the college of Agriculture. Results show that the OCT setup has a penetration depth of up to $1.5 \mathrm{~mm}$. The work did not show strong correlation between OCT measurement signatures and meat tenderness. The work helped to understand the OCT measurement and clearly pointed out the value of increased measurement speed using swept wavelength OCT and the potential use of SGDBR lasers as the swept wavelength source. One of the conclusions drawn from this application of OCT measurements is that the process can be improved using a faster measurement technique. 
The thesis then studies the characteristics of the SGDBR laser and how they map into the characteristics needed for swept-wavelength OCT applications. A major part of the work was design of both chip-level and package-level sources that were used to evaluate the laser characteristics. Specific properties of this SGDBR laser are measured: wavelength tuning characteristics, optical laser linewidth, amplitude modulation speed, frequency modulation speed and wavelength switching speed on each of the control inputs to the SGDBR device In the end, it is shown that SGDBR lasers can improve the wavelength ramp speeds in OCT. Device concerns include the laser linewidth and its limitations for swept wavelength OCT. This work provided the basis for other graduate students to build up a more complete implementation of an OCT measurement system. 


\section{ACKNOWLEDGMENTS}

First, I would like to thank my thesis advisor, Dennis Derickson, for all of the support he has provided. This thesis would not have been possible without his help and the wealth of information he contributed.

I would also like to thank Andrew DeKelaita for providing plenty of ideas as we worked together over the summer, helping me overcome many small issues that I encountered.

Other contributors were Mike Bernacil for constructing the bases for the chip lasers used and Matt Schlutz for creating the measurement setup that was used for OCT measurements of meat samples.

JDS Uniphase provided the packaged lasers that were used for a successful setup, and Leif Johansson from UC Santa Barbara provided several chip lasers to study; none of this work would have been possible without the lasers they provided.

I would like to thank Professors Xiaomin Jin and John Saghri for being on my thesis committee.

Finally, I would like to thank my family for supporting me during my time at Cal Poly. 


\section{TABLE OF CONTENTS}

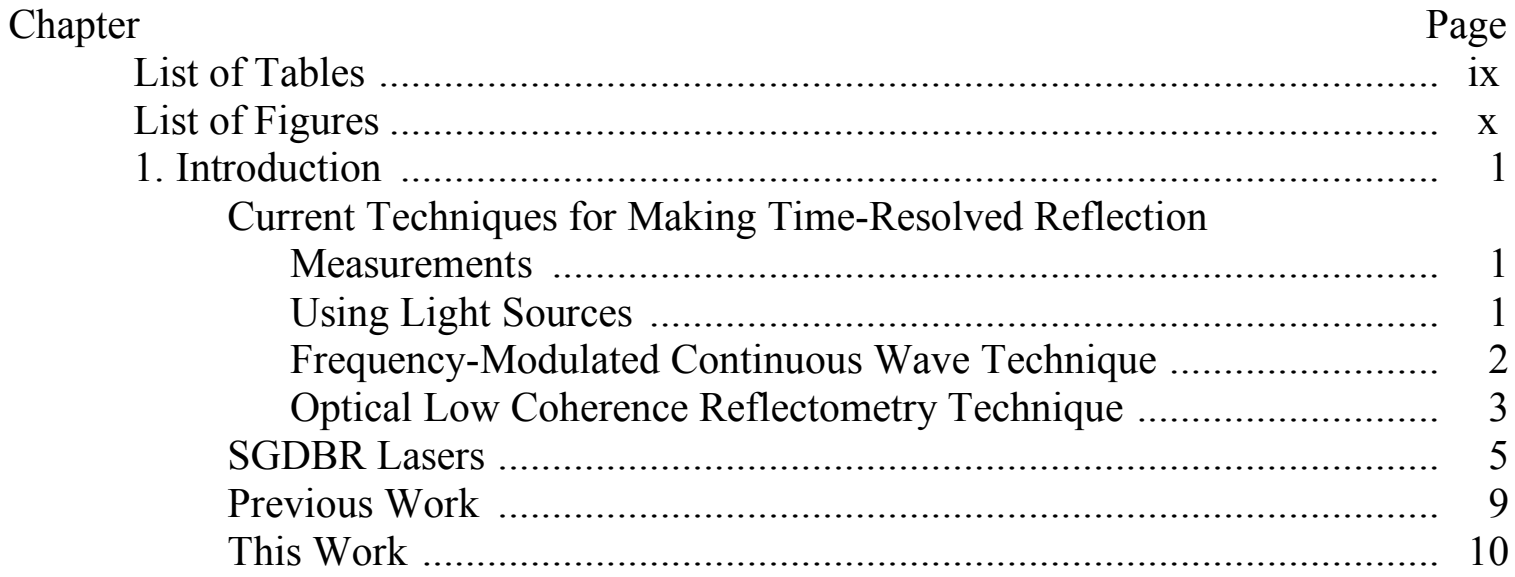

2. White-Light Interferometry Application Example: OCT

Measurements of Meat Tenderness ….................................................. 12

Example of OCT Operation .......................................................... 12

Background on Meat Tenderness Measurement Techniques ................ 12

OCT Measurement Set-up Description ......................................... 13

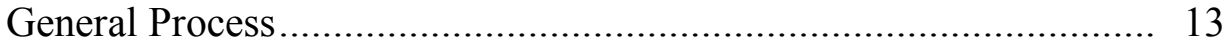

Overall Optical Setup ............................................................... 14

Choosing the Wavelength ....................................................... 16

Computer Control .......................................................... 17

Characterizing the Setup ............................................................ 18

Gaussian Light Pattern ................................................... 18

Penetration Depth ................................................................ 23

Relationship Between Measurements and Meat Tenderness ................ 26

Conclusions ................................................................................ 31

Improvements From Other Sources .......................................... 32

3. Chip-level Investigation of the SGDBR Laser ......................................... 33

Description of the SGDBR Chip Laser ............................................ 33

Package Construction ........................................................... 34

Verifying That the Lasers Work ..................................................... 37

Bench Setup ............................................................................... 39

Optical Setup ............................................................................. 40

Lens Pitch ..................................................................... 40

Lens Configuration Testing .................................................... 43

Visual Observations of Laser Output ...................................... 45

DC Wavelength Tuning Efficiency Measurements ............................ 46

Next Steps for Using Chip Lasers ............................................... 47 
4. High Speed Packaged SGDBR Lasers …………................................... 48

Custom-Packaged SGDBR Lasers ................................................. 48

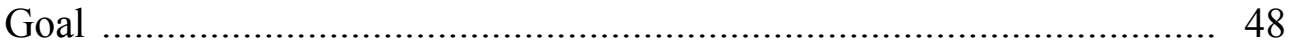

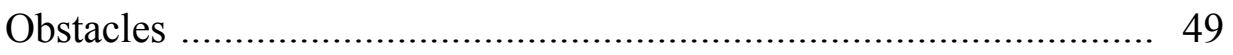

Constructing the Laser Driver Circuitry ............................................... 49

Laser DC Characterization ............................................................. 53

Amplitude Modulation Performance ................................................. 57

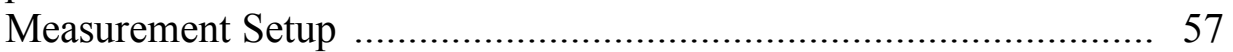

Frequency Modulation Performance ..................................................... 60

Viewing the Frequency Modulation Response on the OSA ……....... 60

Measurement Setup ................................................................ 63

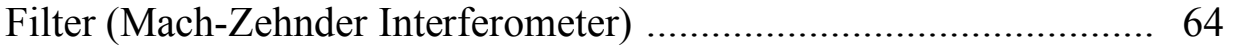

Choosing a Bias Point .................................................................... 66

Modulation Measurement Results ........................................................ 69

Modulation Results Summarized ................................................. 73

Network Analyzer Input Impedance Measurements .............................. 73

Linewidth Measurements ............................................................. 79

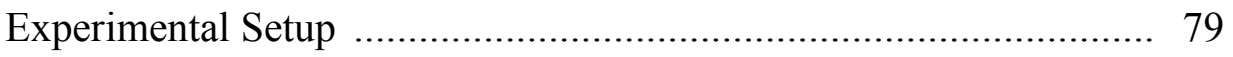

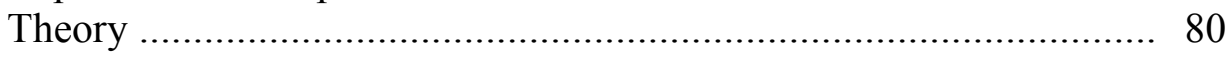

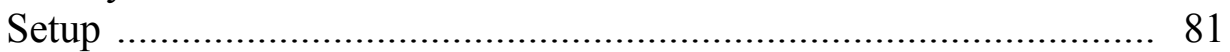

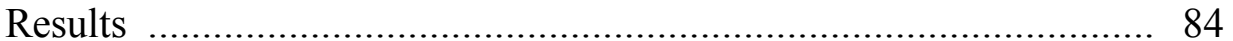

Fixed Wavelength DFB Laser Linewidth ................................ 84

Nortel Tunable Laser (VCSEL) Linewidth .................................. 85

Bookham Laser Linewidth .................................................... 85

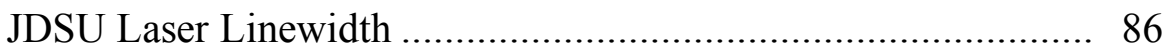

Summary of Linewidth Measurements .................................... 88

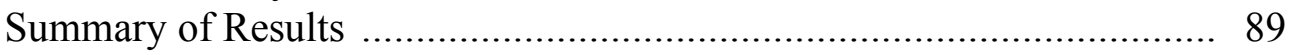

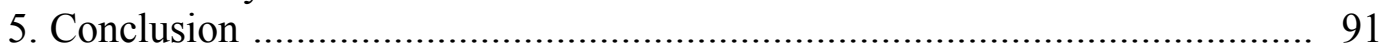

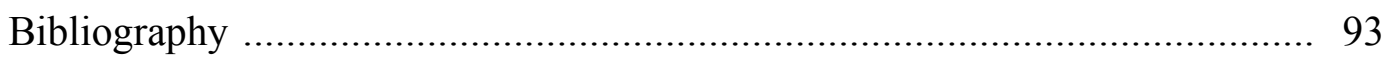

Appendix A: Frequency Modulation Data ……………................................... 95

Appendix B: LabView Program for Collecting Data from ESA …………....... 97

Appendix C: Wavelength vs. Phase Current for Chip Laser ............................... 100

Appendix D: Pin Layout of the Packaged SGDBR laser ................................... 101

Appendix E: Amplitude Modulation Data …………...................................... 102 


\section{LIST OF TABLES}

Table 3.1 Maximum safe current values for the different laser components ................ 34

Table 3.2 Data showing the functionality of the lasers .............................................. 38

Table 4.1 DC bias currents used for wavelength-controlling segments ...................... 68

Table 4.2 Laser segment response times ..................................................................... 73

Table 4.3 Summary of time domain responses for different laser inputs ..................... 78

Table 4.4 Estimated circuit values for the laser inputs ………………..................... 79

Table 4.5 Linewidth measurements for the Nortel tunable laser ................................ 85

Table 4.6 Summary of linewidth measurements .................................................... 88

Table 4.7 Summary of measurements characterizing laser inputs .............................. 90 


\section{LIST OF FIGURES}

Figure 1.1a - Block diagram for FMCW measurement setup .............................. 2

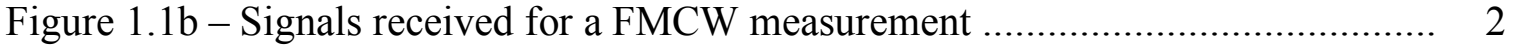

Figure 1.2a - Block diagram for OLCR measurement ........................................ 4

Figure $1.2 b$ - Typical measurement from an OLCR setup .................................... 5

Figure 1.3 - Physical layout of an SGDBR laser ............................................... 6

Figure 1.4 - Reflectivity vs. Wavelength for an SGDBR laser ............................... 8

Figure 1.5 - Spectral Plot of an SGDBR laser .................................................. 9

Figure 2.1 - Pictures of the OCT measurement setup ..................................... 15

Figure 2.2 - Lens setup for making OCT measurements on meat samples ................. 15

Figure 2.3 - Absorption vs. wavelength for several biological materials .................. 17

Figure 2.4 - OCT measurement from a meat sample ............................................ 18

Figure 2.5 - Lens setup for OCT measurement of a meat sample ............................. 19

Figure 2.6 - Plot of all of the reflections in the OCT meat measurement system ........ 20

Figure 2.7 - Measurement setup for finding the distance of maximum

reflection power ...................................................................... 20

Figure 2.8 - Reflected power vs. distance for OCT setup .................................. 21

Figure 2.9 - Setup for finding the beam waist size ............................................... 23

Figure 2.10 - Method used for determining penetration depth ............................... 25

Figure 2.11 - Number of samples at each penetration depth .................................. 25

Figure 2.12 - Light penetration depth vs. lens-meat distance .............................. 26

Figure 2.13 - Slopes of OCT measurements vs. WBS values ................................. 27

Figure $2.14-\mathrm{R}^{2}$ value for OCT measurements vs. WBS values ............................. 28

Figure 2.15 - Example linear and jagged plots of OCT measurements on meat .......... 29

Figure 2.16 - Slopes of OCT measurements vs. WBS values for high $\mathrm{R}^{2}$ values ........ 30

Figure 2.17 - Penetration depth of OCT measurement vs. WBS value ..................... 31

Figure 3.1 - Close-up picture of the chip laser used .............................................. 34

Figure 3.2 - Base used for the chip laser ..................................................... 35

Figure 3.3 - Chip laser being mounted onto the base ..................................... 36

Figure 3.4 - Wire bond connections being created for the laser .......................... 37

Figure 3.5 - Schematic for checking that the lasers work .................................... 38

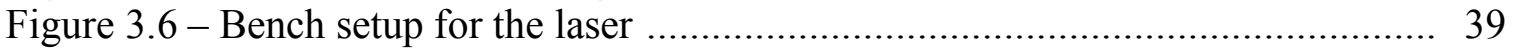

Figure 3.7 - Close-up picture of the lens setup and the laser .............................. 40

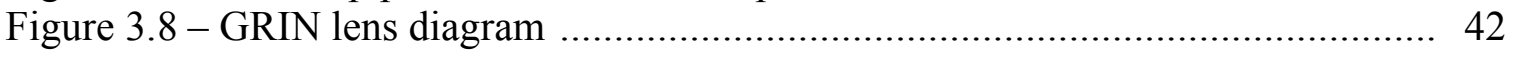

Figure 3.9 - Lens setup aligned to the side of the laser ....................................... 44

Figure 3.10 - Third lens setup used ............................................................... 44

Figure 3.11 - Light modes from the chip laser .................................................... 46

Figure 3.12 - Wavelength versus phase current for the chip laser .......................... 47

Figure 4.1 - Demonstration of pre-emphasis ...................................................... 49

Figure 4.2 - Side view of the connection between a semi-rigid cable and a butterfly lead .......................................................................... 50

Figure 4.3 - Setup for the packaged laser ...................................................... 52 
Figure 4.4 - Block diagram for DC characterization of the SGDBR laser ................. 53

Figure 4.5 - Wavelength as a function of back mirror current ................................ 54

Figure 4.6 - Power as a function of back mirror current ........................................ 54

Figure 4.7 - Wavelength as a function of front mirror current ................................. 55

Figure 4.8 - Power as a function of front mirror current ..................................... 55

Figure 4.9 - Wavelength as a function of phase current ..................................... 56

Figure 4.10 - Power as a function of phase current ............................................... 56

Figure 4.11 - Setup for measuring the amplitude modulation response .................... 57

Figure 4.12 - Input signal for the section being modulated ................................... 58

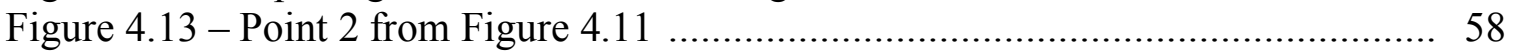

Figure 4.14 - Output from the photodiode (amplitude modulation measurement) ...... 59

Figure 4.15 - Photodiode output (amplitude modulation measurement) .................... 59

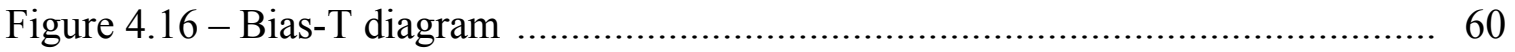

Figure 4.17 - Characterization of the amplifier used ......................................... 60

Figure 4.18 - Setup for observing the effects of frequency modulation ..................... 61

Figure 4.19 - Laser output from $143 \mathrm{kHz}$ modulation of phase section ..................... 62

Figure 4.20 - Laser output from $50 \mathrm{MHz}$ modulation of phase section ...................... 62

Figure 4.21 - Setup for frequency modulation measurements .............................. 63

Figure 4.22 - Mach-Zehnder interferometer ................................................ 65

Figure 4.23 - Mach-Zehnder interferometer being used as a wavelength

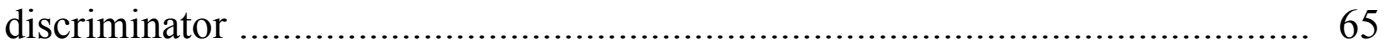

Figure 4.24 - Picking a DC bias point for the phase section .................................. 67

Figure 4.25 - Picking a DC bias point for the front mirror section .......................... 67

Figure 4.26 - Picking a DC bias point for the back mirror section .......................... 68

Figure 4.27 - Amplitude modulation of Gain section ........................................... 69

Figure 4.28 - Amplitude modulation of SOA section .......................................... 69

Figure 4.29 - Amplitude modulation of the front mirror ...................................... 70

Figure 4.30 - Frequency modulation of the front mirror .................................... 70

Figure 4.31 - Amplitude modulation of the back mirror ....................................... 71

Figure 4.32 - Frequency modulation of the back mirror .................................. 71

Figure 4.33 - Amplitude modulation of the phase section .................................. 72

Figure 4.34 - Frequency modulation of the phase section .................................. 72

Figure 4.35 - Setup for simulating time domain responses from the laser inputs ........ 74

Figure 4.36 - Circuit model of the laser inputs ............................................ 74

Figure 4.37 - Time domain response, phase segment ........................................ 75

Figure 4.38 - Time domain response, front mirror segment ................................ 76

Figure 4.39 - Time domain response, back mirror segment ............................... 77

Figure 4.40 - Input into the laser segments .............................................. 78

Figure 4.41 - Combining a signal with itself to measure linewidth .......................... 81

Figure 4.42 - Block diagram for measuring linewidth .................................. 82

Figure 4.43 - Linewidth measurement for the DFB laser ..................................... 84

Figure 4.44 - Linewidth measurement for the Bookham laser ............................... 86

Figure 4.45 - Linewidth measurement for the JDSU laser, front mirror bias ............. 87

Figure 4.46 - Linewidth measurement for the JDSU laser, back mirror bias ............. 87

Figure 4.47 - Linewidth measurement for the JDSU laser, phase bias ...................... 88 
CHAPTER 1: Introduction

\subsection{Current Techniques for Making Time-Resolved Reflection Measurements}

\subsubsection{Using Light Sources}

Multiple techniques exist for using light to make time-resolved reflection measurements of objects. One of these techniques is known as LIDAR (Light Detection and Ranging), which works on the same basic principle as RADAR. Light is sent out, it bounces off of objects, and the time delay between the pulse being sent and the reflected signal being received is translated into a distance. RADAR uses frequencies in the radio and microwave ranges, which works well for many uses including finding enemy aircraft. However, the wavelengths used, which go down to about $4 \mathrm{~mm}$, do not reflect well from nonmetallic surfaces such as atmospheric particles.

This is where LIDAR comes in. This technique uses light in the visible, UV, or near-infrared ranges to make measurements. The shorter wavelength of the light used allows the measurement of atmospheric particles, which can be used to determine weather conditions, among other things [12].

Another reflection measurement method that has developed is Optical Coherence Tomography (OCT), which is able to make measurements with a spatial resolution of less than $2 \mu \mathrm{m}$. This method is similar to LIDAR in the fact that it sends out a light signal and measures the return signal. It also uses the same general frequency range. However, instead of measuring the time delay between sending the pulse and receiving the return signal, it works by comparing the received signal with a reference signal. 


\subsubsection{Frequency-Modulated Continuous Wave Technique}

There are several techniques for making OCT measurements. One technique is called frequency-modulated continuous wave (FMCW). This method uses a narrow-band light source. The laser performs a linear ramp of frequency versus time. Figure 1.1a shows a block diagram of an FMCW measurement, and Figure 1.1b shows the signals received from a sample measurement.

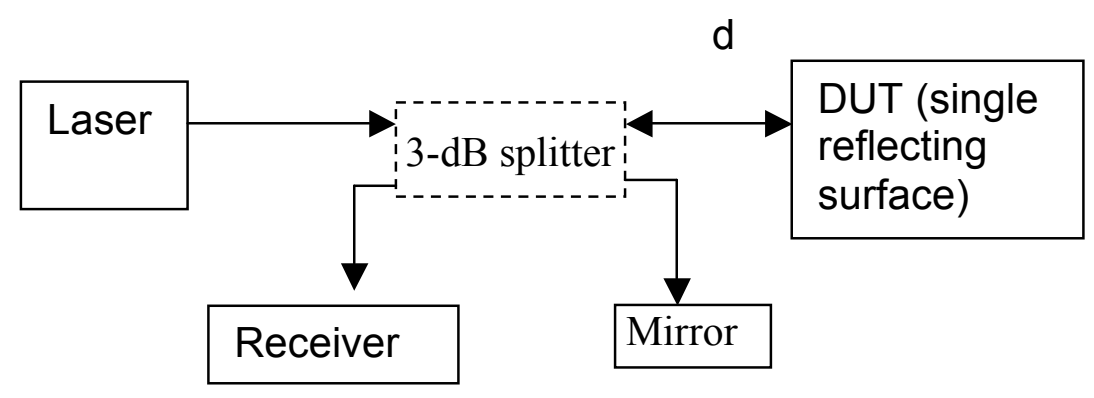

Figure 1.1a: Block diagram for FMCW measurement setup

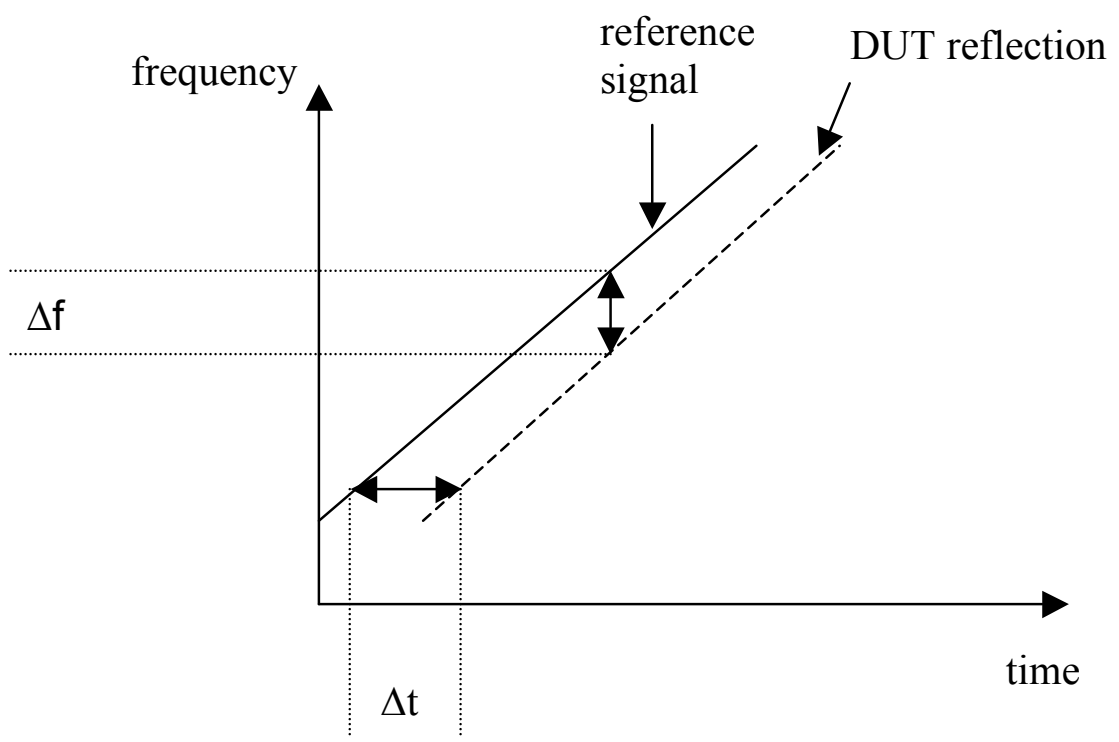

Figure 1.1b: Signals that arrive at the receiver for an FMCW measurement 
The signal coming from the laser increases in frequency at a constant rate, creating a ramp of frequency versus time. This ramp is also reflected off of the DUT, with a time delay $\Delta \mathrm{t}$. This time delay is caused by the difference in distances between the path with the DUT and the path without the DUT. This time delay causes a frequency difference $\Delta \mathrm{f}$ between the two signals when they arrive at the receiver. When the reflected signals arrive at the photodetector, they combine to create a single signal at the difference frequency. By taking the Fourier transform of this combined signal, a plot of received power versus frequency can be made. The frequency axis can be changed to time based on the slope of the frequency ramp, and that can be changed to distance based on the speed of light in the medium.

\subsubsection{Optical Low Coherence Reflectometry Technique}

Another technique is called optical low coherence reflectometry (OLCR), which is also commonly referred to as white light interferometry. In this method, a broadspectrum light source is split into two paths - the reference path and the measurement path. The reference path has a movable mirror, while the device under test (DUT) is held stationary. Light reflects off of the mirror, and it also reflects off of parts of the DUT. Light is combined from both paths, and the time-averaged version of the received power is measured. If the distance from the source to the mirror is equal to the distance from the source to a reflective portion of the DUT, then the reflected signals will interfere. If the distances are unequal, then the interference will be random and result in only a DC signal, which can be filtered out [3]. 
In the simple case where the DUT is a single reflective surface, a signal will be picked up only when the distance to the reference mirror is nearly the same as the distance to the DUT. This generates a signal around a single position. The plot obtained will show photocurrent versus time, where time can be replaced with distance based on the movement rate of the reference mirror. Figure 1.2a shows the setup for this measurement method, and Figure 1.2b shows a typical measurement.

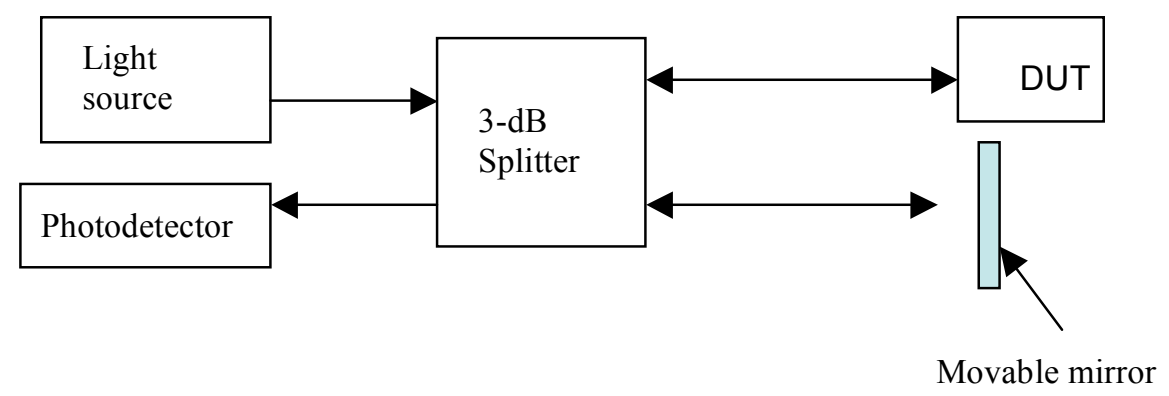

Figure 1.2a: Block diagram for OLCR measurement 


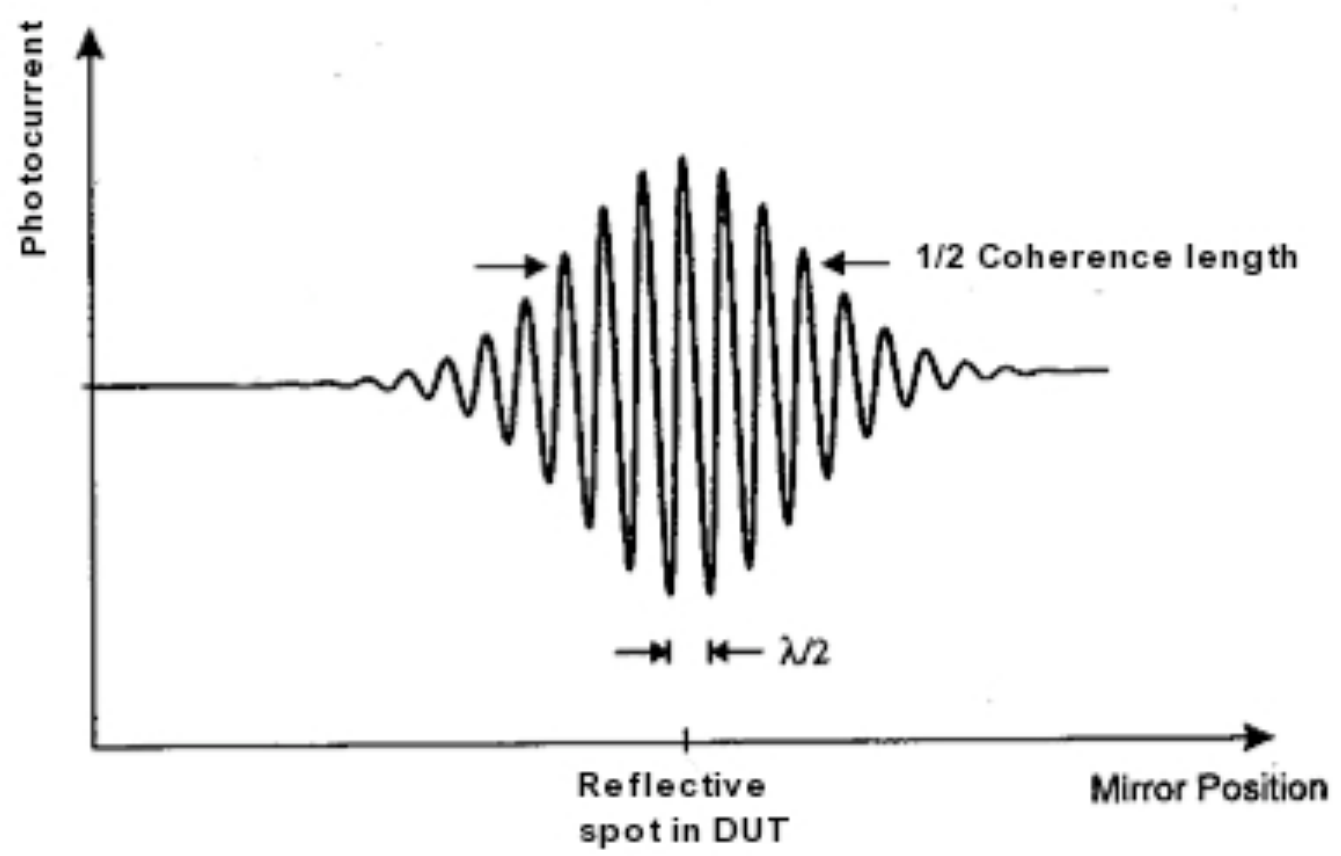

Figure 1.2b: Typical measurement from an OLCR setup where the DUT is a single reflective surface. The signal varies sinusoidally due to a changing phase difference between the two electric fields. The envelope decreases as the coherence between the fields decreases.

\subsection{SGDBR Lasers}

The goal of this work is to show that Sampled Grating Distributed Bragg Reflector (SGDBR) lasers can introduce improvements to OCT measurements. The advantage that SGDBR lasers have is that they can switch between different wavelengths very quickly, allowing faster measurements to be made. Previous research has found that SGDBR wavelength switching can be done in under 5 ns [9]. Existing measurement techniques are capable of making measurements at a $16 \mathrm{kHz}$ repetition rate [10]. Using fast wavelength switching, improvements can be made to OCT measurement techniques. 


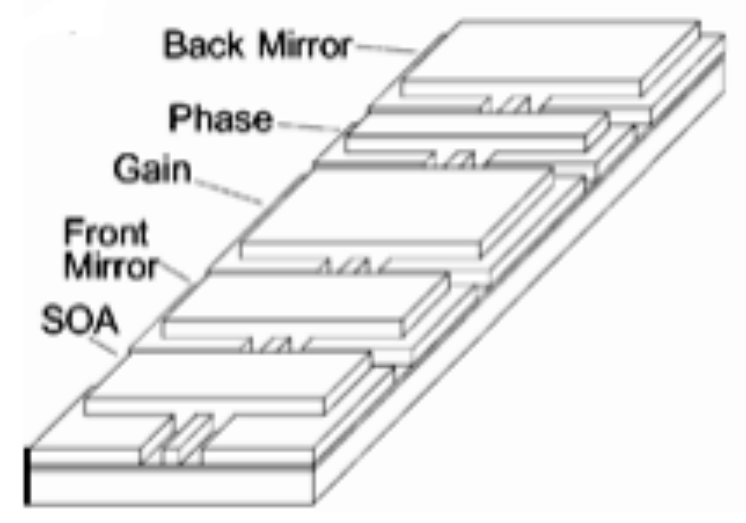

Figure 1.3: Physical layout of an SGDBR laser [9]

Figure 1.3 shows the layout of an SGDBR laser. This laser has several components built onto it. Each of the five segments requires a separate current input. The front mirror and back mirror are used together for large changes in wavelength; the phase is used for small changes in wavelength. The gain and semiconductor optical amplifier (SOA) are used to amplify the light to a usable level.

Photons are initially generated through spontaneous generation of photons in the gain section. When the photons get to the front or back mirror, they are reflected if they are at the right wavelength and transmitted otherwise. Only a single wavelength is reflected from both the front mirror and the back mirror.

Any time a photon goes through the gain section it causes stimulated emission of other photons. The new photons have the same wavelength, direction and phase as the photon that stimulated them. By having photons bouncing back and forth many times through the gain section, inducing more stimulated photons each time, a large amount of light can be produced at that one wavelength. A small amount of light passes through the 
front mirror instead of being reflected or absorbed, and this light is amplified once more in the SOA before leaving the laser. Figure 1.4 shows how the front and back mirrors control the wavelength.

For each mirror, there are a series of equally spaced peaks that represent highly reflective wavelengths. The spacing for the two mirrors is shown by the equation in

$$
\Delta \lambda=\frac{\lambda^{2}}{2 n L}
$$

In (1.1), $\Delta \lambda$ is the spacing between the peaks, $\lambda$ is the current operating wavelength, $\mathrm{n}$ is the index of refraction for the material, and $\mathrm{L}$ is the spacing between the mirrors. The operating wavelength occurs where both curves show reflection. When the current is changed for a mirror, the entire reflectivity curve shifts left or right for that mirror, causing a jump in wavelength to a different spike on the other curve. For example, in Figure 1.4, the first spike lines up on each curve. If the back mirror current is changed such that the curve shifts to the right, then the second spike on the two curves would quickly line up. Figure 1.5 shows the actual output from an SGDBR laser at several different wavelengths. 

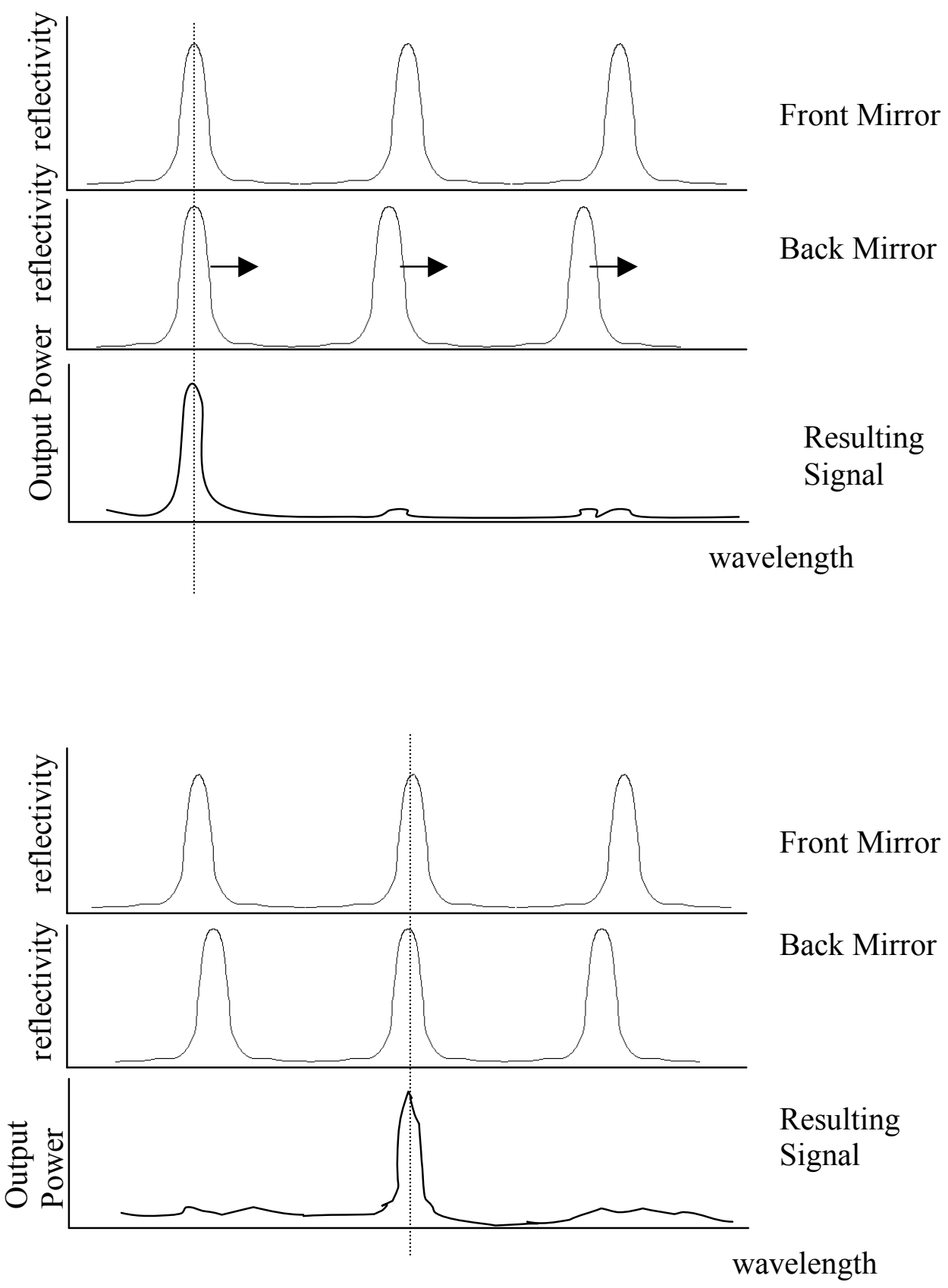

Figure 1.4: Graphs showing potential reflection versus wavelength patterns for the front and back mirrors. 


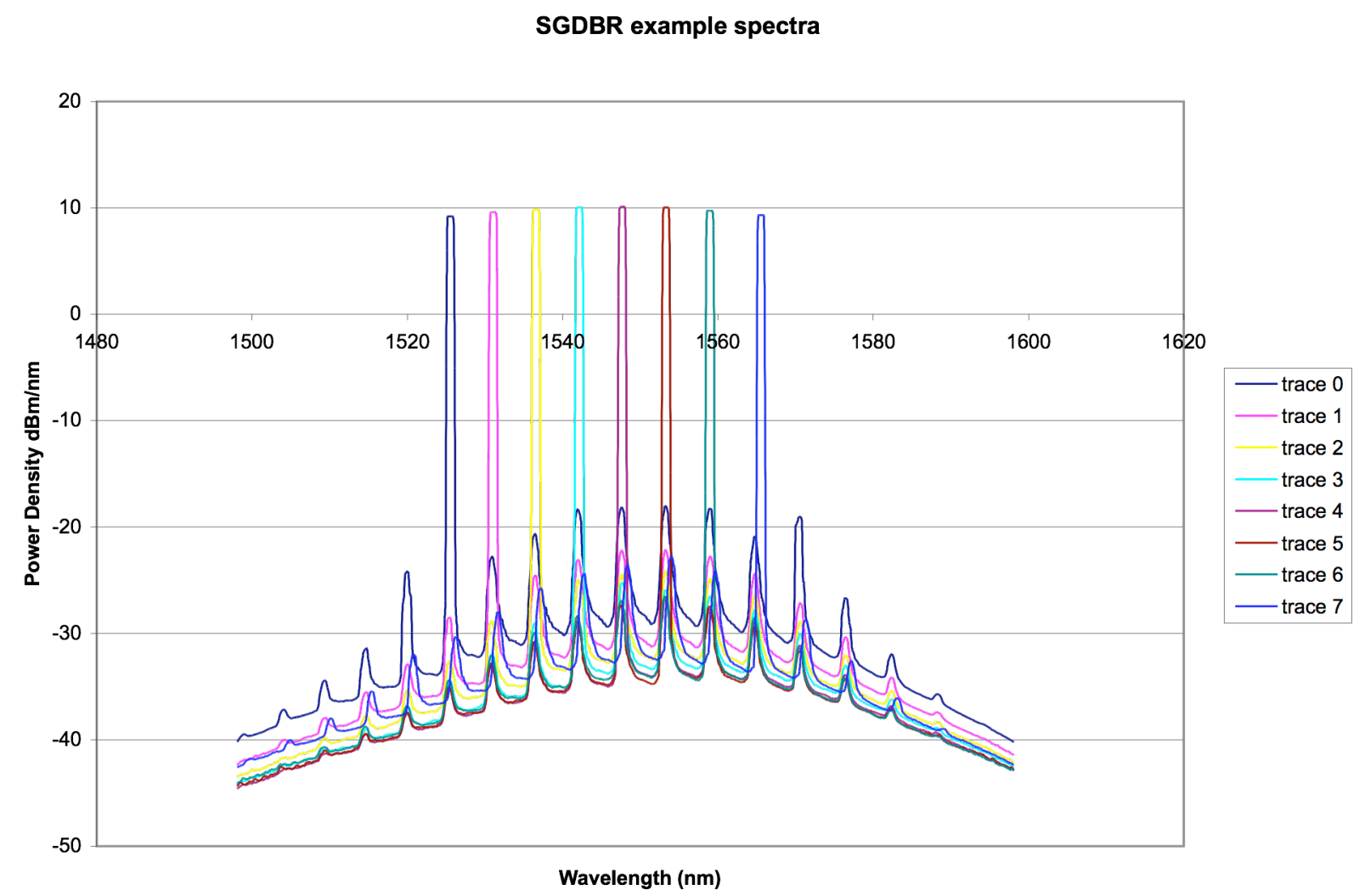

Figure 1.5: Spectral plot of an SGDBR laser with several different input currents. When one of the input currents is changed the laser output changes to the next mode.

\subsection{Previous Work}

Andrew DeKelaita did work to identify the DC tuning characteristics of these SGDBR lasers (DeKelaita). He looked at tuning the front mirror and back mirror to achieve different wavelengths. Some of the current combinations gave inconsistent wavelength results due to hysteresis. (Hysteresis is the condition where changing to a set of DC inputs did not always produce the same wavelength output if the previous state of the laser wasn't the same between the measurements.) These unreliable input combinations were discarded, and the remaining input combinations were examined. Using different combinations of input currents, he found that it is possible to obtain any 
desired output wavelength, and he found a set of input currents to increase the output wavelength by constant step sizes.

\subsection{This Work}

Andrew DeKelaita's research explored the DC tuning capability of the laser. This report will explore the high speed modulation and linewidth characteristics of the laser. One goal is to determine exactly how fast the laser can change wavelengths. Another goal is to construct a package that would allow the high-speed switching that is expected from the laser to be achieved. A few different setups were explored to see how fast they can be operated.

The first setup explored is a chip-level package. This setup has the laser (approximately $4 \mathrm{~mm}$ long, $1 \mathrm{~mm}$ wide, and less than $0.5 \mathrm{~mm}$ tall) mounted on a pad (approximately $5 \mathrm{~mm} \times 7 \mathrm{~mm}$ ). This pad has several bonding pads to be used for creating electrical connections. The main benefit of using the chip laser is that all of the connections can be controlled, which allows the parasitic effects between the current sources and the laser to be minimized.

The other SGDBR laser used was a fully packaged laser with electrical leads already prepared to make connections with, as well as a fiber pigtail already attached. JDSU, the company that donated this laser, didn't design the package for high-speed switching, which was a drawback. However, they removed the low-pass filters that were in place for each of the inputs, allowing for faster input switching. The main advantage to this setup is that the laser itself was all ready to use, eliminating the need to create the wire bond connections directly to the fragile laser. This also made the setup less fragile; 
the first chip laser became damaged due to being tapped by a magnifying glass while examining it.

The rest of this paper will discuss the capabilities of these two laser setups. The details of each setup will be discussed, along with the efforts to maximize performance. Finally, the results will be given and explained. First, however, a current OCT technique is investigated to get a better understanding of the type of measurement being made. This will allow a comparison to be made between the measurement techniques used currently and proposed setups using SGDBR lasers. 


\section{CHAPTER 2: White-Light Interferometry Application Example: OCT Measurements of Meat Tenderness}

\subsection{Example of OCT Operation}

This chapter will give an overview of the process of making OCT measurements with the purpose of learning more about an end application of fast wavelength tuning SGDBR lasers. This will be done by covering the OCT measurements that were made for a particular application - meat tenderness testing. In the process, another goal is to find an improved method for making a measurement that currently does not use any optical systems.

\subsection{Background on Meat Tenderness Measurement Techniques}

The current method of measuring meat tenderness is a relatively slow process. One method of making this measurement is known as the Warner-Bratzler Shear Force Measurement [11]. First, the meat must be cooked using precise methods. This first requires that the meat be cut into uniform pieces, often done by freezing the meat and cutting it into solid pieces. Next, the meat has to be thawed to a temperature of 2 to $5^{\circ} \mathrm{C}$. After that, the meat is cooked in a controlled manner so that the internal temperature of multiple pieces will consistently reach $71^{\circ} \mathrm{C}$. (The core temperature of meat continues to rise after it is removed from the heat, so there is more involved than simply removing the meat when $71^{\circ} \mathrm{C}$ is reached.) This is followed by chilling the meat overnight. Several pieces of meat are then cored out of the main piece. The actual measurement is made using a specific blade cutting into the cored pieces of meat, and the force required to cut 
into the meat is called the WBS value, where a larger value indicates that the meat is less tender than a piece with a lower WBS value.

This measurement takes about a day. The meat must be refrigerated for many hours (for thawing and again for cooling). A carefully controlled cooking setup must be maintained. A precise coring and cutting process must be followed. This process is time consuming and takes multiple steps that must follow strict guidelines. A reduction in time required or in specific procedures to follow would make the process run more smoothly. OCT techniques were used to see if a correlation between the reflectivity profile and the meat tenderness could be established. There are three goals for this process. The first goal is to describe the method using OCT measurements and how it works. The second goal is to determine if a correlation can be found between the OCT data and the meat tenderness values determined by industry-accepted techniques, since this would be necessary for OCT techniques to replace the currently used technique. The third goal is to learn more about the OCT application in anticipation of building a sweptwavelength OCT system with SGDBR lasers.

\subsection{OCT Measurement Set-up Description}

\subsubsection{General Process}

Before describing the details of the OCT measurement setup, a general overview

of the process is needed. The measurements were taken on three separate days. For each day of measurements, the meat was first cooled by people in the Animal Science Department, led by Professor Robert Delmore, in preparation for being cooked. Some samples were picked up for the day (before they were cooked) and transported to the optics lab to work with using a cooler. OCT measurements were made and the meat 
samples were returned to the Animal Science Department. Then they proceeded to cook the meat and continue with their system for measuring WBS. Each piece of meat had a card with it to identify it by number, which allowed the OCT measurements to be linked with the data collected by the Animal Science Department.

All of the meat that was used for these measurements was New York Steak, which is the meat between the lower ribs and pelvis. Fourteen meat samples were used the first day, 16 on the second day, and 20 for the third day. For each piece of meat, three separate measurements were made from different parts of the meat.

\subsubsection{Overall Optical Setup}

The 8504B Precision Reflectometer was used to make measurements. This instrument, made by Agilent, uses white-light interferometry (covered in Section 1.1.2) to make the OCT measurement. The Reference Extension ports were connected together, and the lens and setup was connected to the Test Port. That setup consisted of an optical fiber with a pigtailed ferrule at the end of it, with a graded index (GRIN) lens in front of that. Figure 2.1 shows pictures of the OCT measurement setup, and Figure 2.2 shows a diagram of the lens configuration.

The lens setup has to be constructed properly to make sure that the light coming from the sample focuses on the lens. A cylindrical tube is used to hold the ferrule lens and GRIN lens in the proper alignment. The lens setup was attached to an XYZ stage so that it could be moved around above the meat sample. 


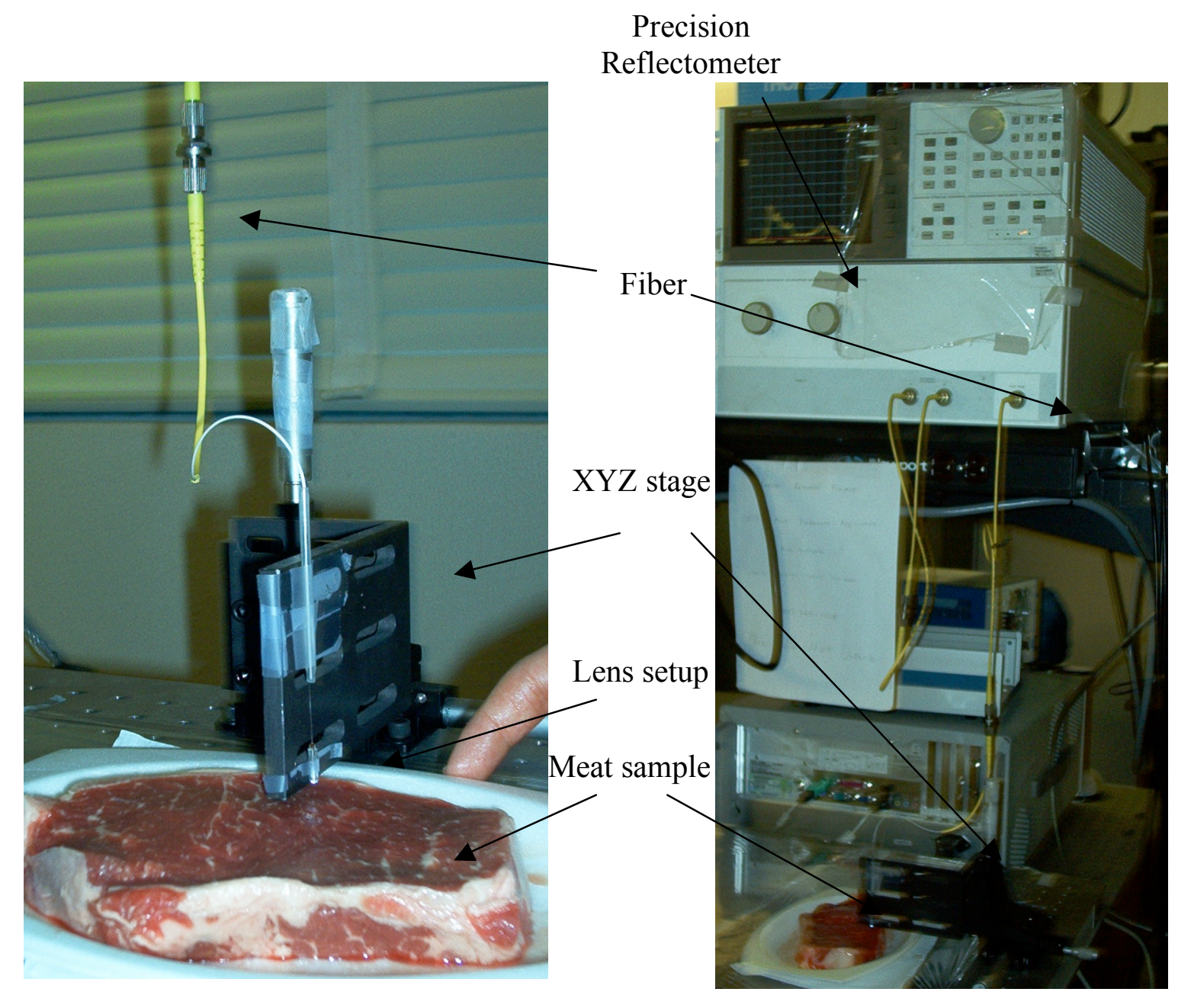

Figure 2.1: Pictures of the OCT measurement setup. The picture on the left is a close-up picture of the XYZ stage over a meat sample. The picture on the right shows the entire setup.

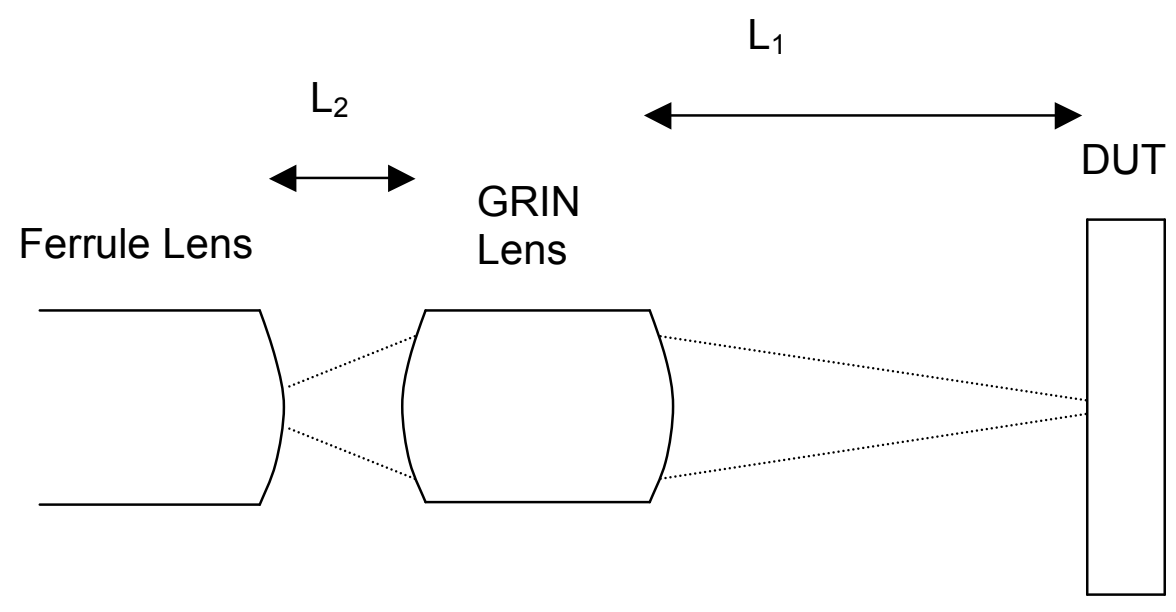

Figure 2.2: Lens setup for making OCT measurements on meat samples. $\mathrm{L}_{2}=$ $0.675 \mathrm{~mm}, \mathrm{~L}_{1}$ is adjustable. 


\subsubsection{Choosing the Wavelength}

The 8504B Precision Reflectometer can operate around either $1300 \mathrm{~nm}$ or 1550 nm. It is important to use a wavelength that will be able to best penetrate into the meat. For this, research done by Alan McWilliam was referenced. The experiments done by him test how well several wavelengths $(532,800,1064$, and $1278 \mathrm{~nm})$ penetrate through poultry, and also through rat brain. In both cases, $1278 \mathrm{~nm}$ was able to penetrate much more power through the material, showing that this wavelength worked better than lower wavelengths.

The testing did not use longer wavelengths. However, they also include a graph of absorption versus wavelength of major components of biological materials, shown in Figure 2.3. Melanin and oxygenated hemoglobin decline in absorption as wavelength increases, while water increases in absorption. The absorption of melanin and water flatten out at nearly the same absorption value between $1200 \mathrm{~nm}$ and $1350 \mathrm{~nm}$ before water becomes more absorbing. The higher absorption of water at longer wavelengths means that $1300 \mathrm{~nm}$ is likely a better wavelength for measuring meat tenderness. 


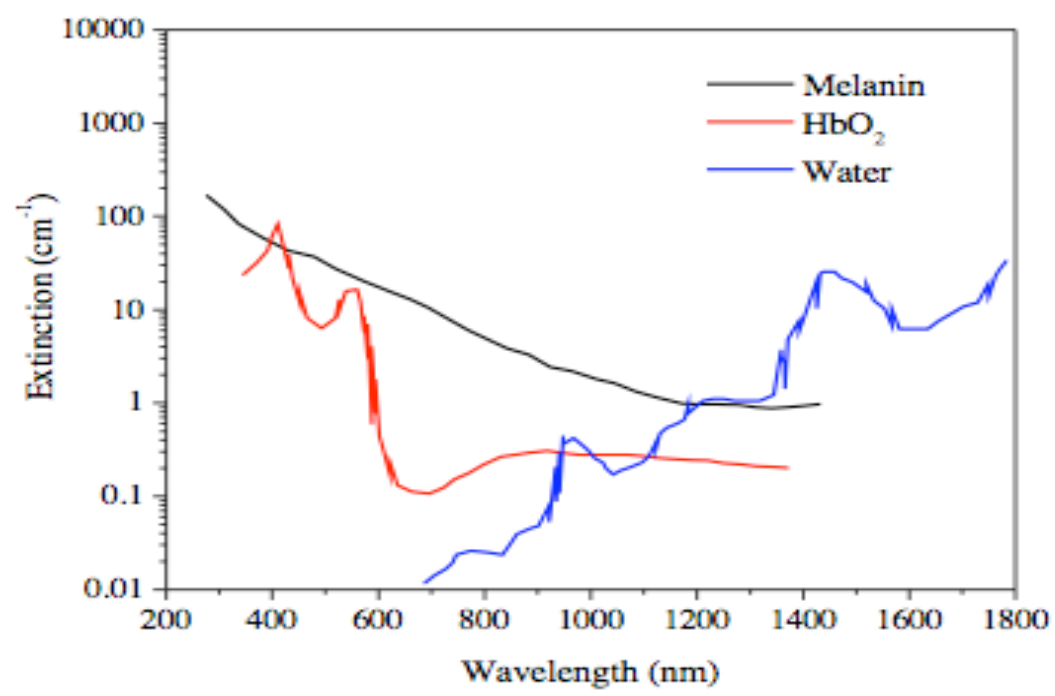

Figure 2.3: Absorption vs. wavelength for several biological materials. Higher absorption means less penetration at that wavelength. The wavelength where all materials have low absorption values is around $1300 \mathrm{~nm}[6]$.

\subsubsection{Computer Control}

To make measurements using the Reflectometer, the first step was to be able to collect the measurement data. To facilitate this, Matt Schlutz created a LabView program that would collect the data from the Reflectometer using a GPIB connection. The measurement settings are entered into the Reflectometer, and then 401 data points are taken from the measurement and recorded. Figure 2.4 shows a sample measurement, where the data has been copied into Excel and turned into a graph. The goal is to find some connection between this data and the meat tenderness. 


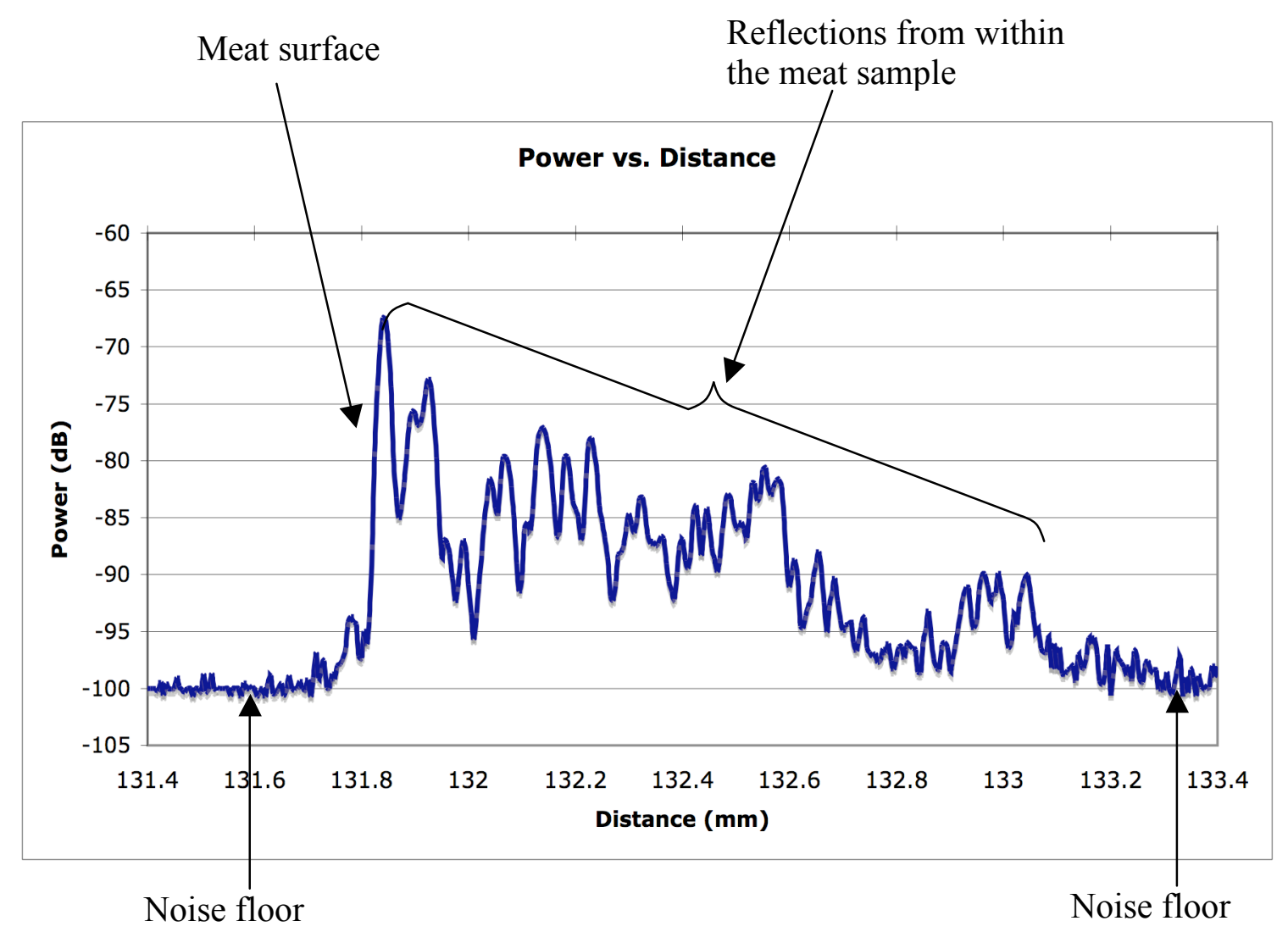

Figure 2.4: OCT measurements from a meat sample. An index of refraction of 1 is assumed for the $\mathrm{x}$-axis. This is not accurate inside the meat, but the actual index of refraction is unknown.

\subsection{Characterizing the Setup}

\subsubsection{Gaussian Light Pattern}

One thing characterized was the pattern of the light coming out from the GRIN

lens. Figure 2.5 shows the expected light output. The ferrule lens and GRIN lens are held together by a cylindrical tube. The light coming out of the GRIN lens converges before diverging again, but it does not converge to a single point.

A few different things were done to check this hypothesis. First, a piece of flat glass was used as the DUT, and reflected power was measured as a function of distance 
from the GRIN lens, shown in Figure 2.7. The peak shows up around $2.9 \mathrm{~mm}$. This should represent the narrowest portion of the beam path, where the maximum amount of power is concentrated in a small spot. With a lens pitch of 0.29 , the expected distance for the peak measurement is $2.87 \mathrm{~mm}$ [8]. A plot of this is shown in Figure 2.8 .
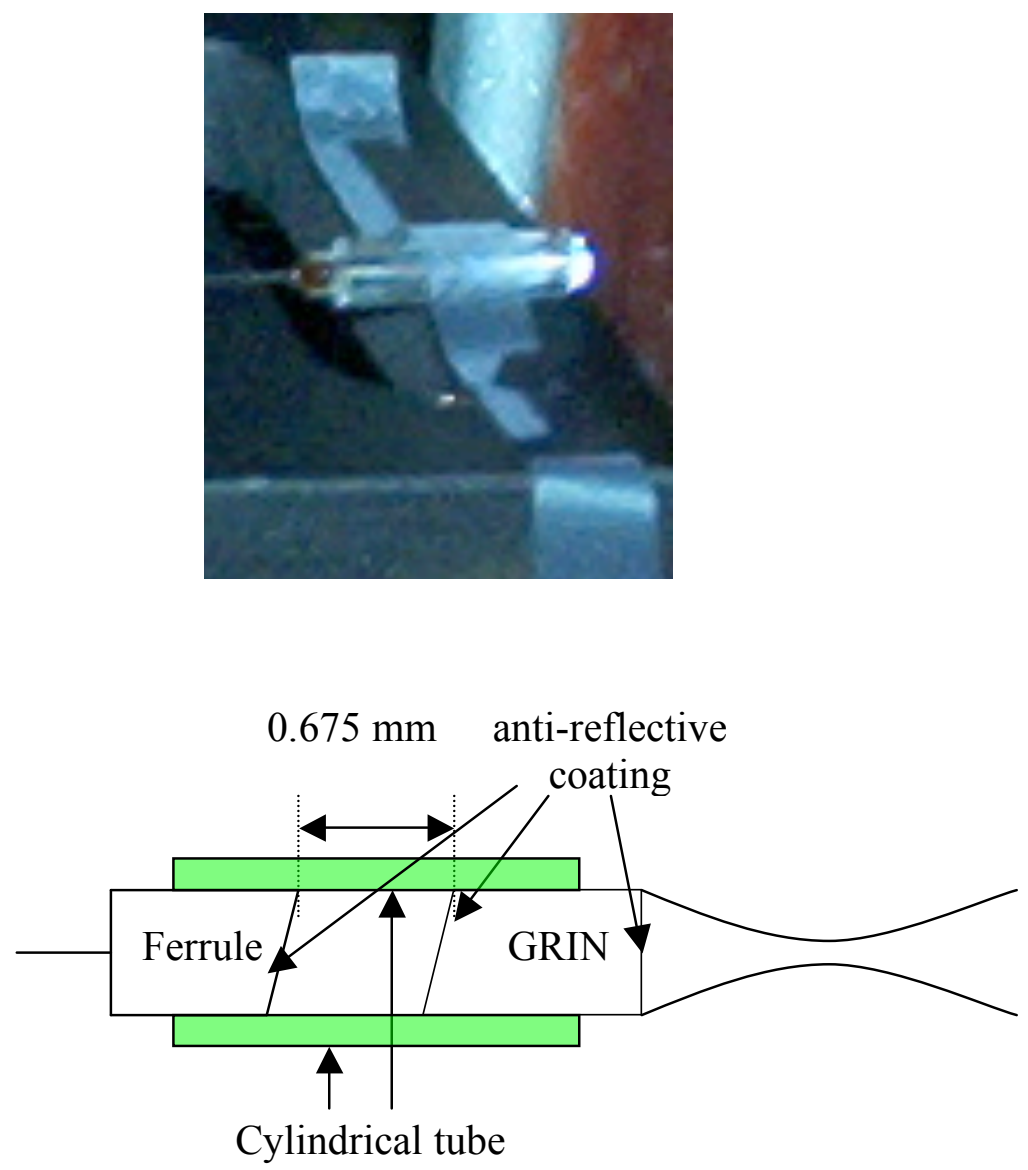

Figure 2.5: Lens setup for OCT measurement of a meat sample. The actual lens setup is shown on the top, and a diagram is shown on the bottom. The picture on the top shows the lens setup attached to an XYZ stage. The diagram on the bottom shows the layout of the lens setup along with the light coming out of it. 


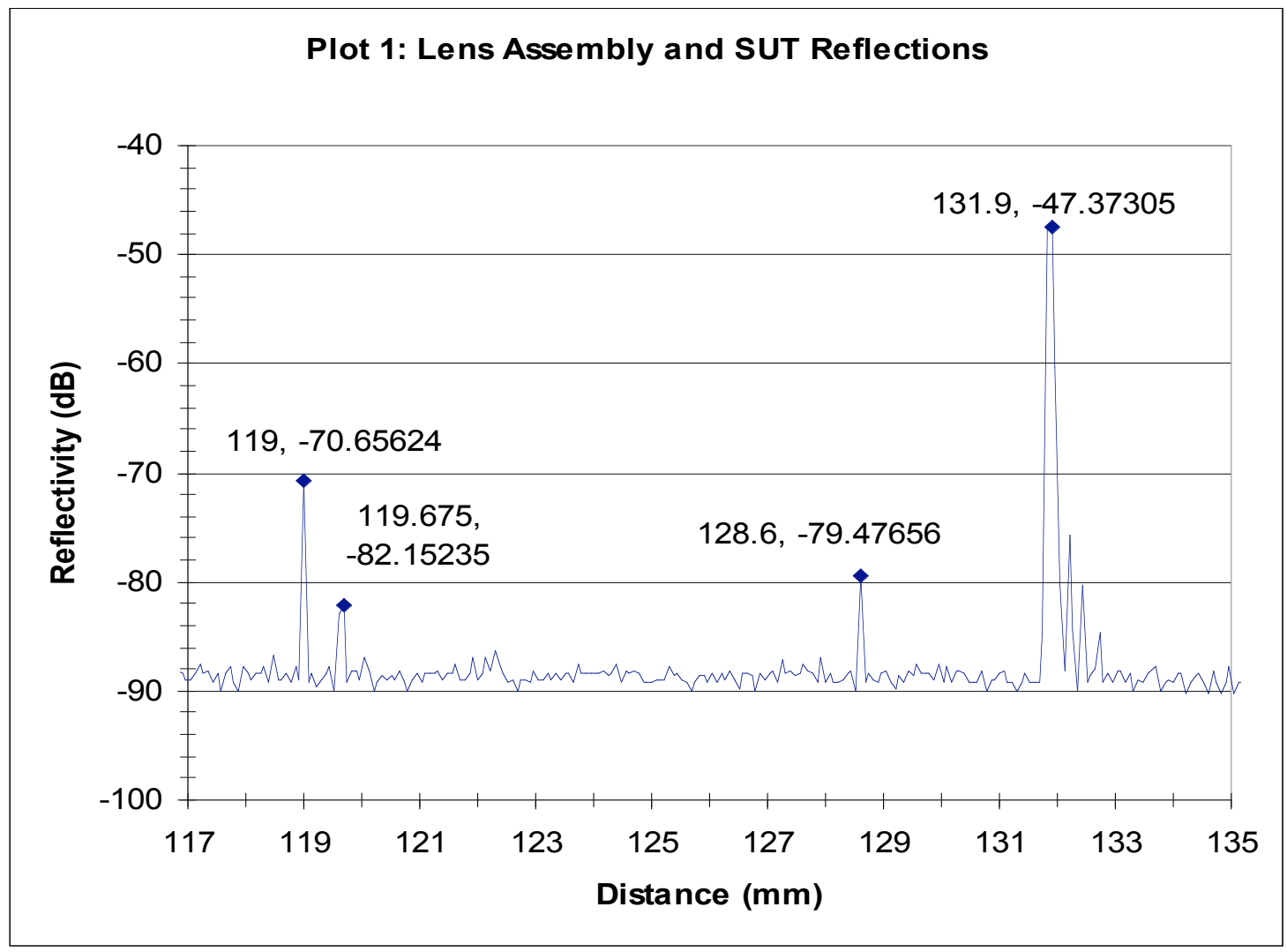

Figure 2.6: Plot of all of the reflections in the OCT measurement system. This plot was obtained by Matt Schlutz, and it shows that the distance between the ferrule lens and the GRIN lens is $0.675 \mathrm{~mm}$.

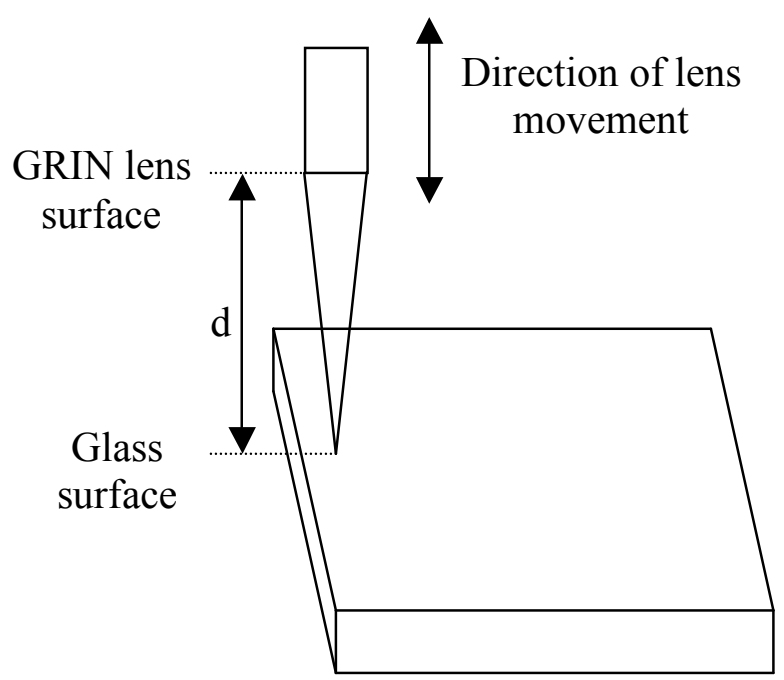

Figure 2.7: Measurement setup for finding the distance of maximum reflection power. 


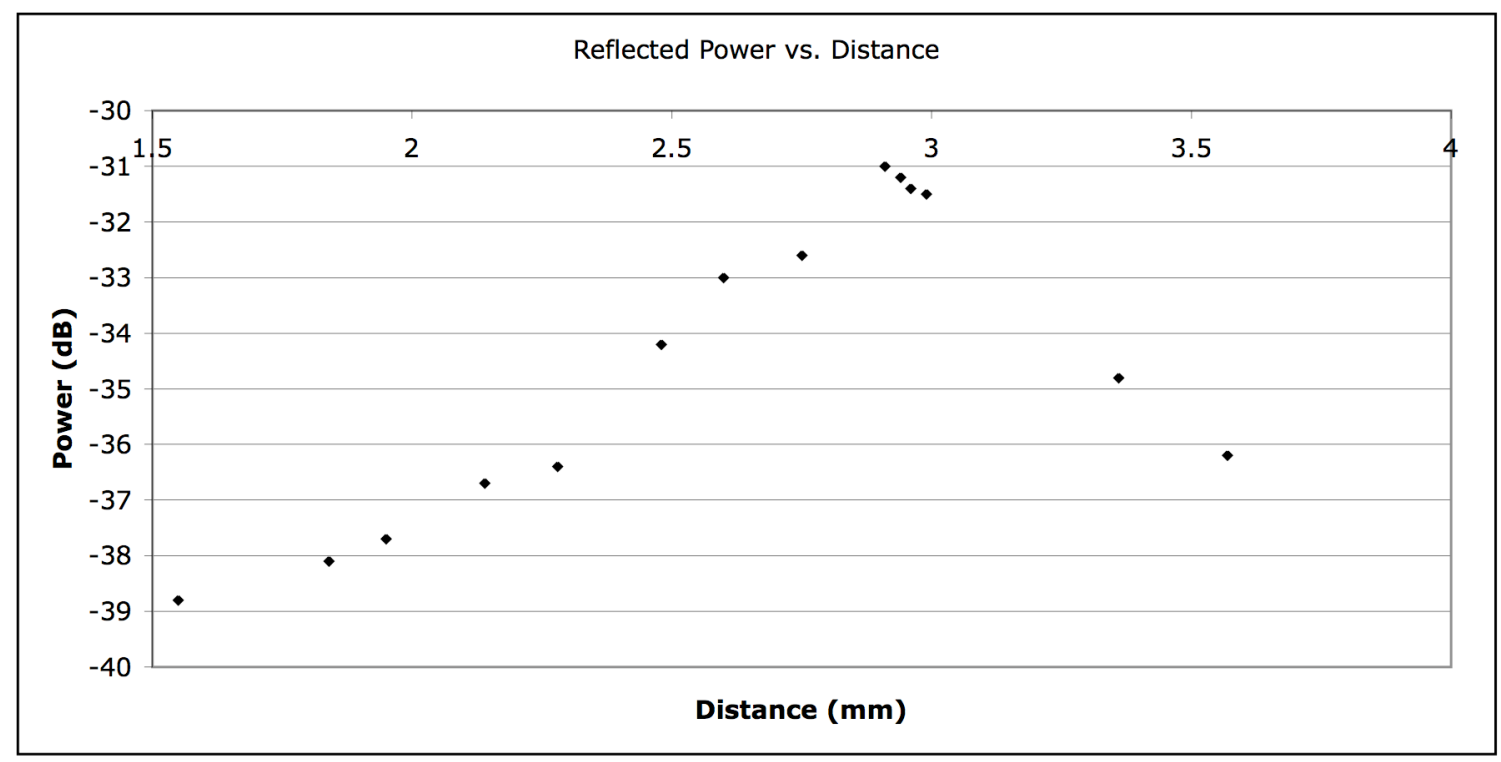

Figure 2.8: Reflected power vs. distance for OCT setup. The reflected power is shown as a function of the distance between the lens and the reflective surface (shown in Figure 2.7), with the peak reflected power representing the location of the narrowest portion of the beam path

The other thing that was measured is the beam waist size at the focal distance. This was done using the same piece of flat glass. The lens setup was arranged over the glass such that it could be moved parallel to the glass surface. First the lens setup was moved up and down to ensure that it was at the position for a maximum power reading. The lens setup was moved sideways toward the edge just until the power started to decrease, which indicated that some of the light was going past the side of the glass instead of hitting it. With that position identified, the lens setup was moved until the reflected power dropped by $3 \mathrm{~dB}$, which indicated that half of the light was going off the side of the glass. This gave the radius for the beam waist. Figure 2.9 shows how this setup works. This measurement showed that the radius for the beam waist was approximately $\mathbf{5} \boldsymbol{\mu m}$. 
This value is then compared with the expected value for the minimum beam waist size. This can be found using the equation $w_{0}=\frac{\lambda f}{\pi w}$, where $\mathrm{w}_{0}$ is the beam waist radius at the focal distance, $\lambda$ is the wavelength of the light, $f$ is the focal distance, and $w$ is the beam waist radius when it leaves the lens [7]. An estimate has to be made for $w$. The diameter of the GRIN lens is $1.8 \mathrm{~mm}$, but not all of that distance is occupied by the beam waist. A beam waist diameter of $1 \mathrm{~mm}$ is assumed, which leads to a beam waist radius of $0.5 \mathrm{~mm}$ when light leaves the lens. Using this, $1.3 \mu \mathrm{m}$ for $\lambda$, and $2.9 \mathrm{~mm}$ for $f, \mathbf{w}_{\mathbf{0}}$ is

\section{$2.4 \mu \mathrm{m}$.}

The value obtained experimentally and theoretically are different by a factor of two. There are several sources for this discrepancy. First, the accuracy of the measurement was limited. Second, the theoretical calculation required an assumption about the beam waist when the beam left the lens. These sources of error lead to the conclusion that the discrepancy is not unreasonable. 


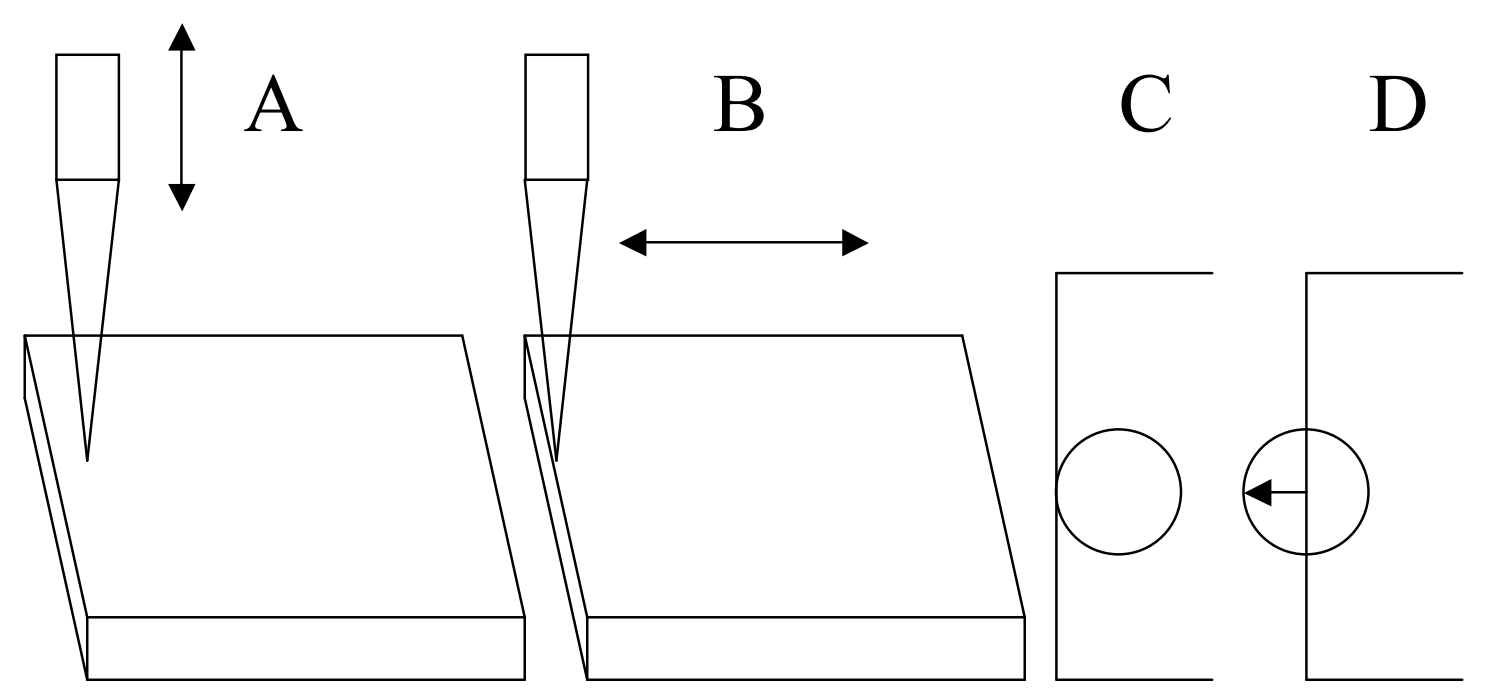

Figure 2.9: Setup for finding the beam waist size. In Figure A, the lens setup is moved up and down to find the distance where maximum power reflection occurs. In Figure B the lens setup is moved toward the edge of the reflective surface, while the vertical distance determined in Figure $\mathrm{A}$ is maintained. Figure $\mathrm{C}$ shows the position of the light beam on the reflective surface, positioned right at the edge of it. Figure D shows the same thing as Figure $\mathrm{C}$, except that the lens setup, and the light beam along with it, have been moved over; when half of the power is reflected, the radius can be determined, as shown by the arrow in Figure D.

\subsubsection{Penetration Depth}

An important thing to know about the setup to make OCT measurements of meat is how deep into the meat the light will be able to penetrate. The penetration depth varied from one sample to another. To determine the penetration depth, the data collected from many samples was examined to find the individual penetration depths. The starting point was taken as the first peak in most cases. In some cases, there was no clear peak for the beginning of the measurement, so the position where the power reached $-80 \mathrm{~dB}$ was used. The end of the measurement was taken to be the deepest location where the power was at $-95 \mathrm{~dB}$. The difference between the two positions was taken to be the penetration depth. 
The method for determining penetration depth is illustrated in Figure 2.10. Figure 2.11 shows how many samples there were at different penetration depths. Out of 89 samples, the minimum was $0.285 \mathrm{~mm}$, the maximum was $1.585 \mathrm{~mm}$, the mean was $0.921 \mathrm{~mm}$, and the median was $0.895 \mathrm{~mm}$.

One factor came to mind that was under controllable in these measurements and could have affected the penetration depth. This factor was the distance between the GRIN lens and the meat surface. Figure 2.12 shows a plot comparing the lens-to-meat distance with penetration depth. This data came from a single day of measurements, using 16 meat samples with three measurements per sample for a total of 48 measurements. This plot does not show any correlation between penetration depth and lens-to-meat distance. 


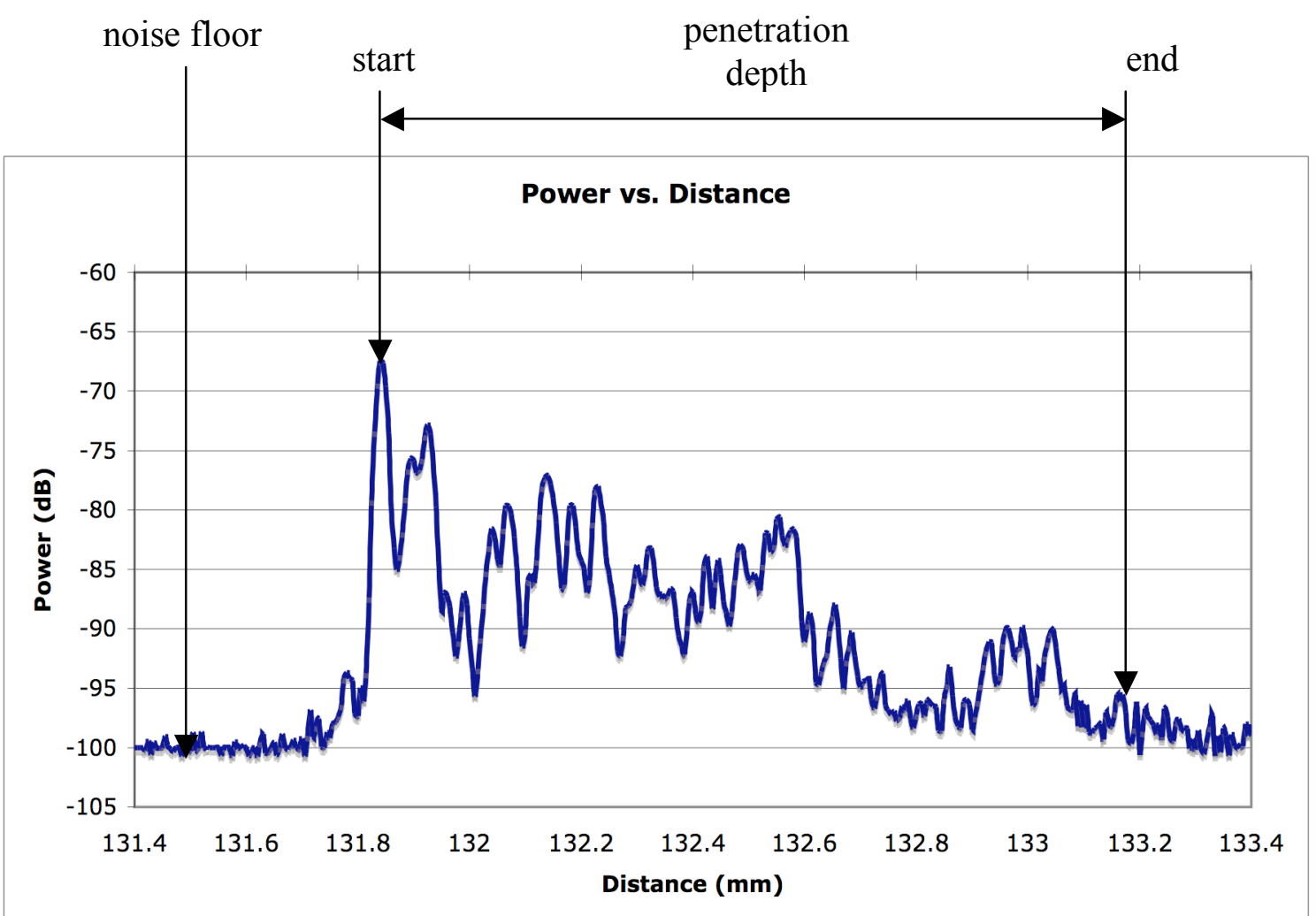

Figure 2.10: Method used for determining penetration depth. An index of refraction of 1 is assumed for the $\mathrm{x}$-axis. This is not accurate inside the meat, but the actual index of refraction is unknown.

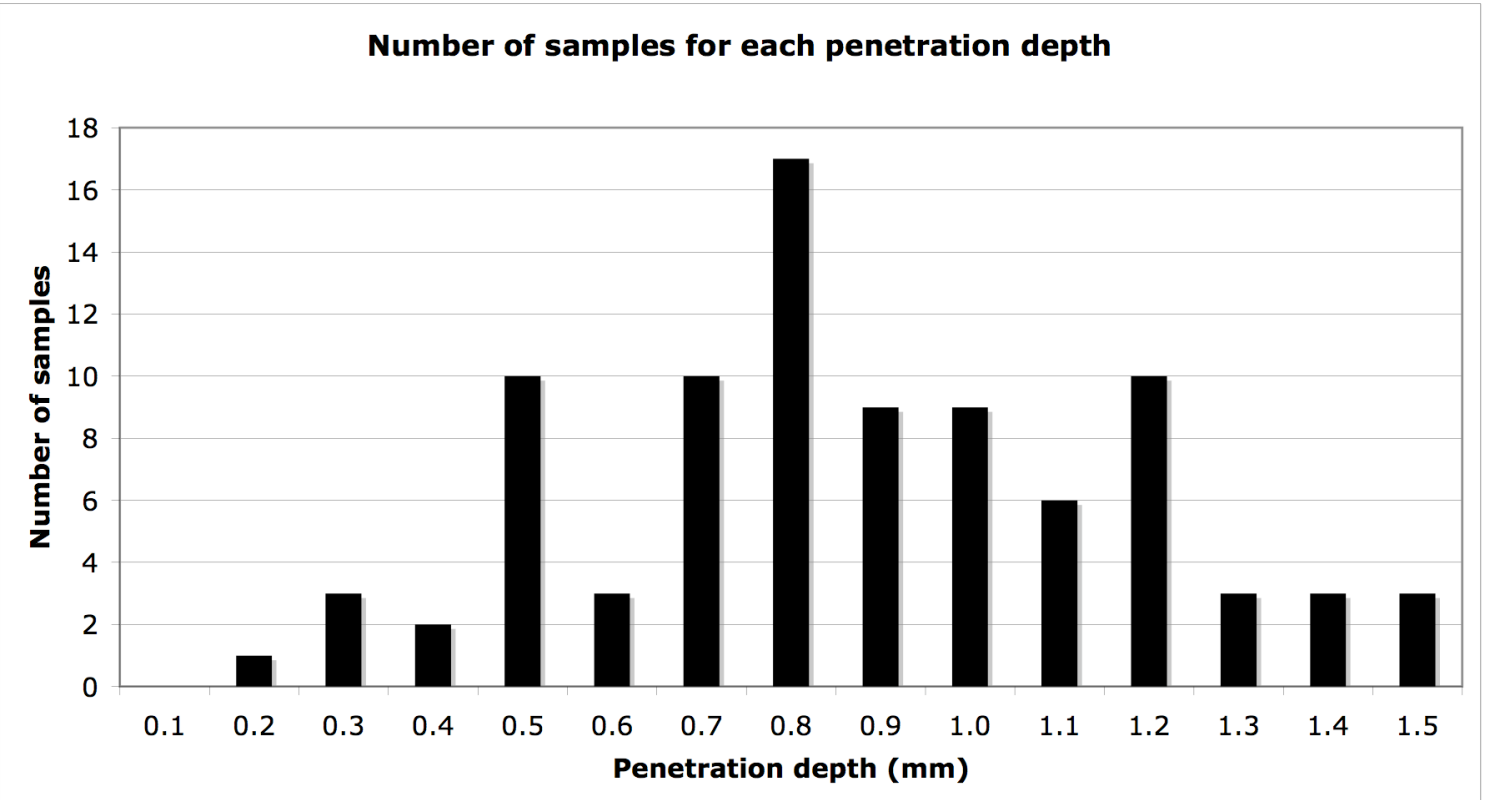

Figure 2.11: Number of samples in each penetration depth grouping; the 0.2 grouping includes everything from 0.2 to 0.2999 , and so on for the other groupings. 


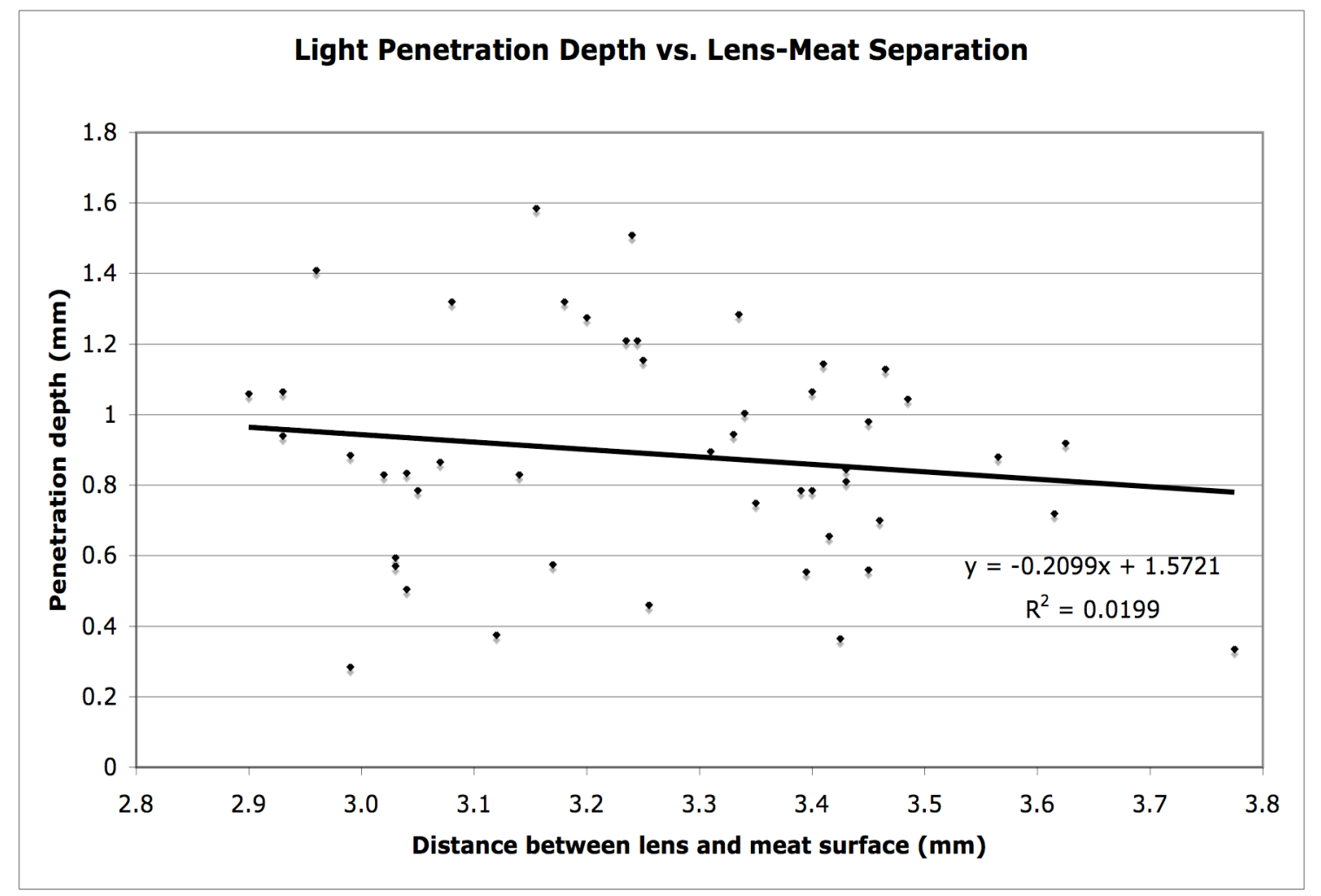

Figure 2.12: Comparison of light penetration depth with the distance between the lens and the meat sample. The shallow slope and the small $\mathrm{R}^{2}$ value (much closer to 0 than to 1 ) indicates that there is no correlation between these variables.

\subsection{Relationship Between Measurements and Meat Tenderness}

The data was analyzed several different ways to attempt to find a relationship between the OCT measurements and the WBS values. The first thing done was to compare the slope of the decay with the WBS value. The decay slope for a measurement is determined by taking the data points starting after the initial spike up to where the measurement has reached the noise floor; with this data, a linear best-fit line was created using Excel. This analysis was done on the data from the first day of measurements, which gave 41 measurements from 14 meat samples. Figure 2.13 shows this comparison. 
Based on this plot, there does not appear to be any correlation between the slope of the data and the WBS value.

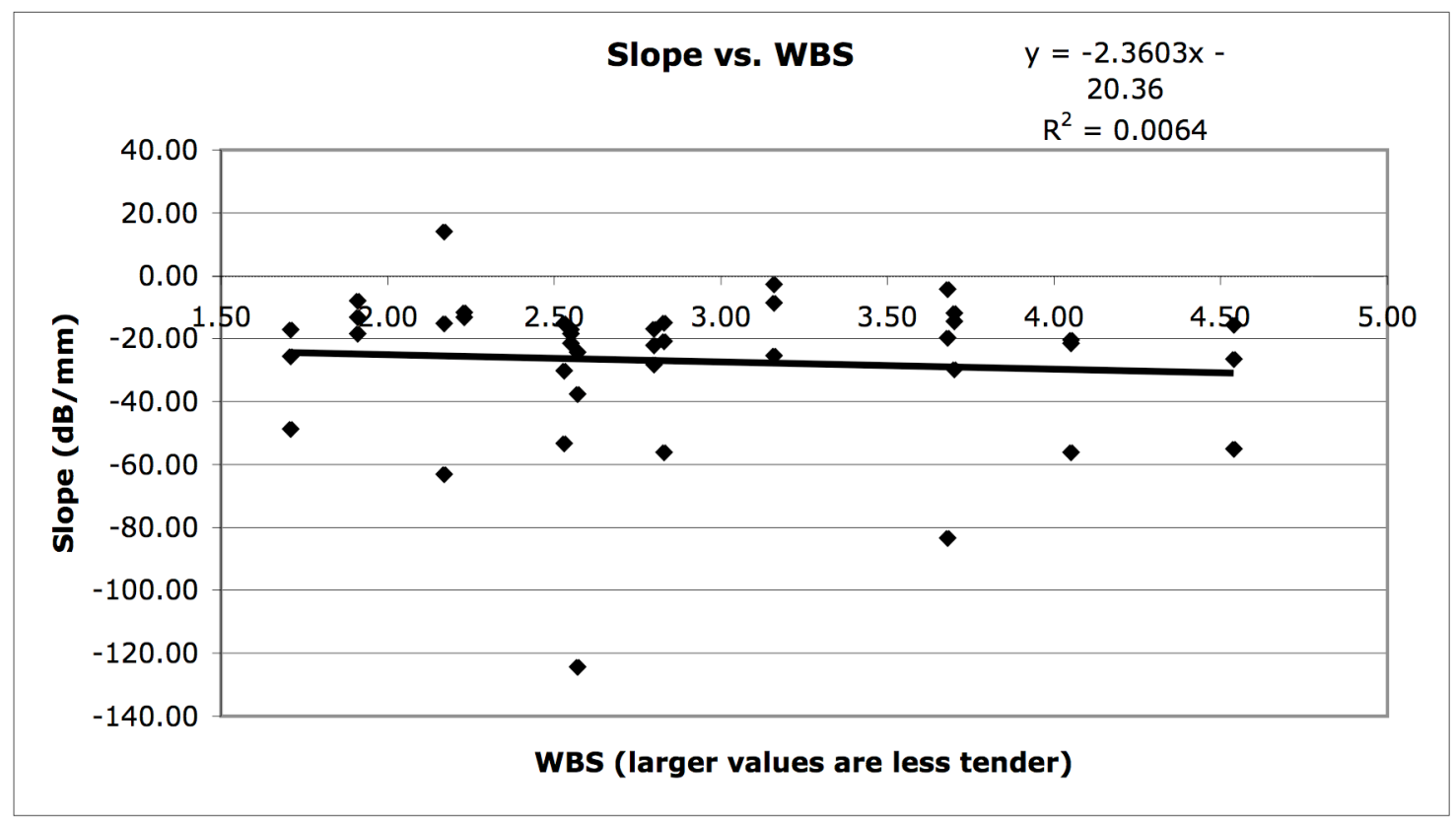

Figure 2.13: Comparison of the slopes of OCT measurements on meat samples with the WBS values for those meat samples, using the data from the first day of measurements. The shallow slope and small $\mathrm{R}^{2}$ value indicate that there is no correlation between these values.

The next comparison was between the $\mathrm{R}^{2}$ value for the line (a value that shows how closely the line fits the data points, with 1 being a perfect fit and 0 meaning it doesn't fit at all) and the WBS value. The $\mathrm{R}^{2}$ value was found at the same time as the slope by using Excel. This comparison tries to identify any relationship between the "jaggedness" of the samples and the WBS values. Figure 2.15 shows a jagged plot and a linear plot. Figure 2.14 has a plot comparing how linear the measurement is to the WBS value for that piece of meat, and it does not show any significant relationship between the values. 


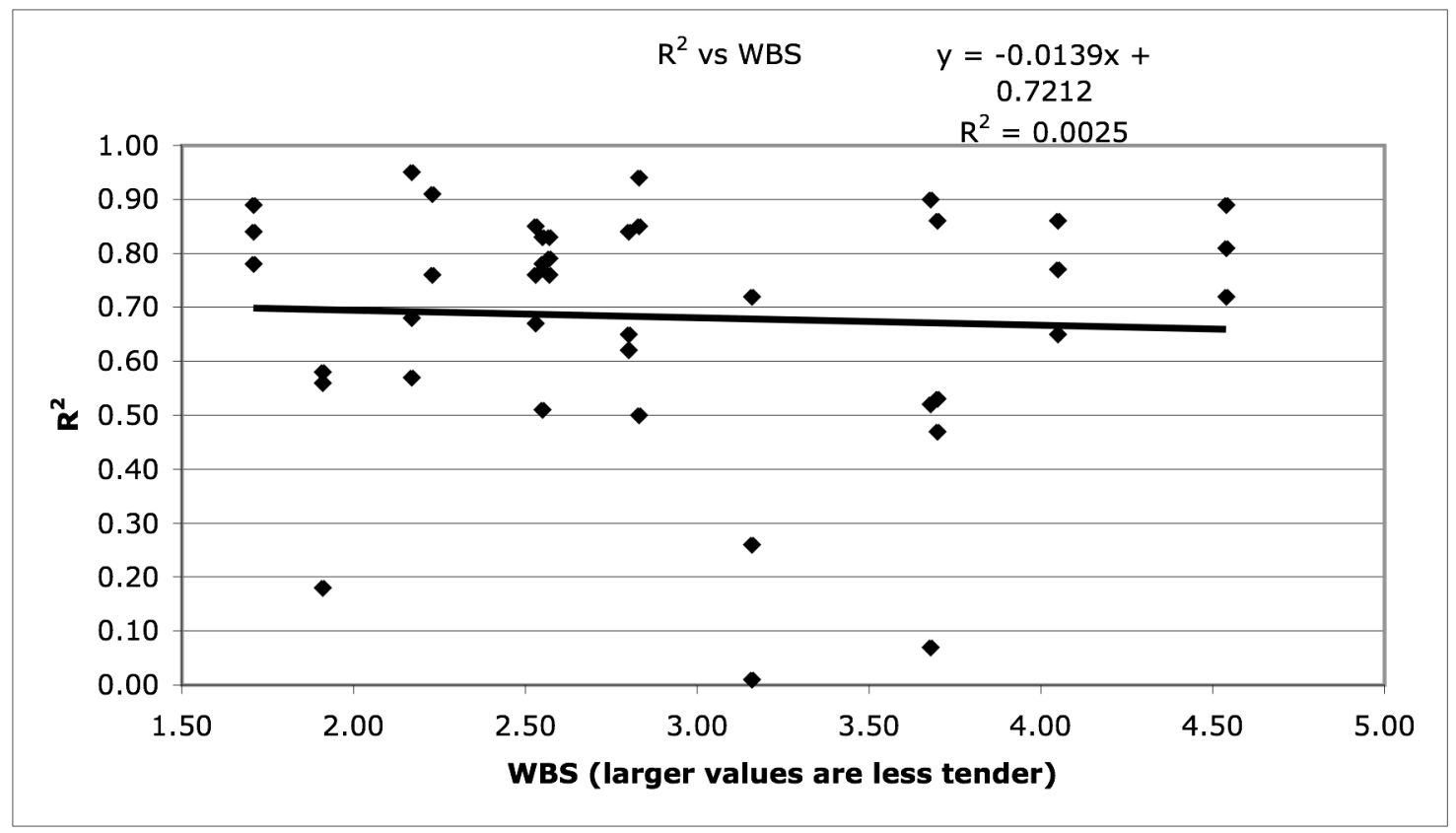

Figure 2.14: Comparison of $\mathrm{R}^{2}$ value for the slope of OCT measurements (representing jaggedness) with WBS values for those samples. The shallow slope and small $\mathrm{R}^{2}$ value indicate that there is no correlation between these values. 

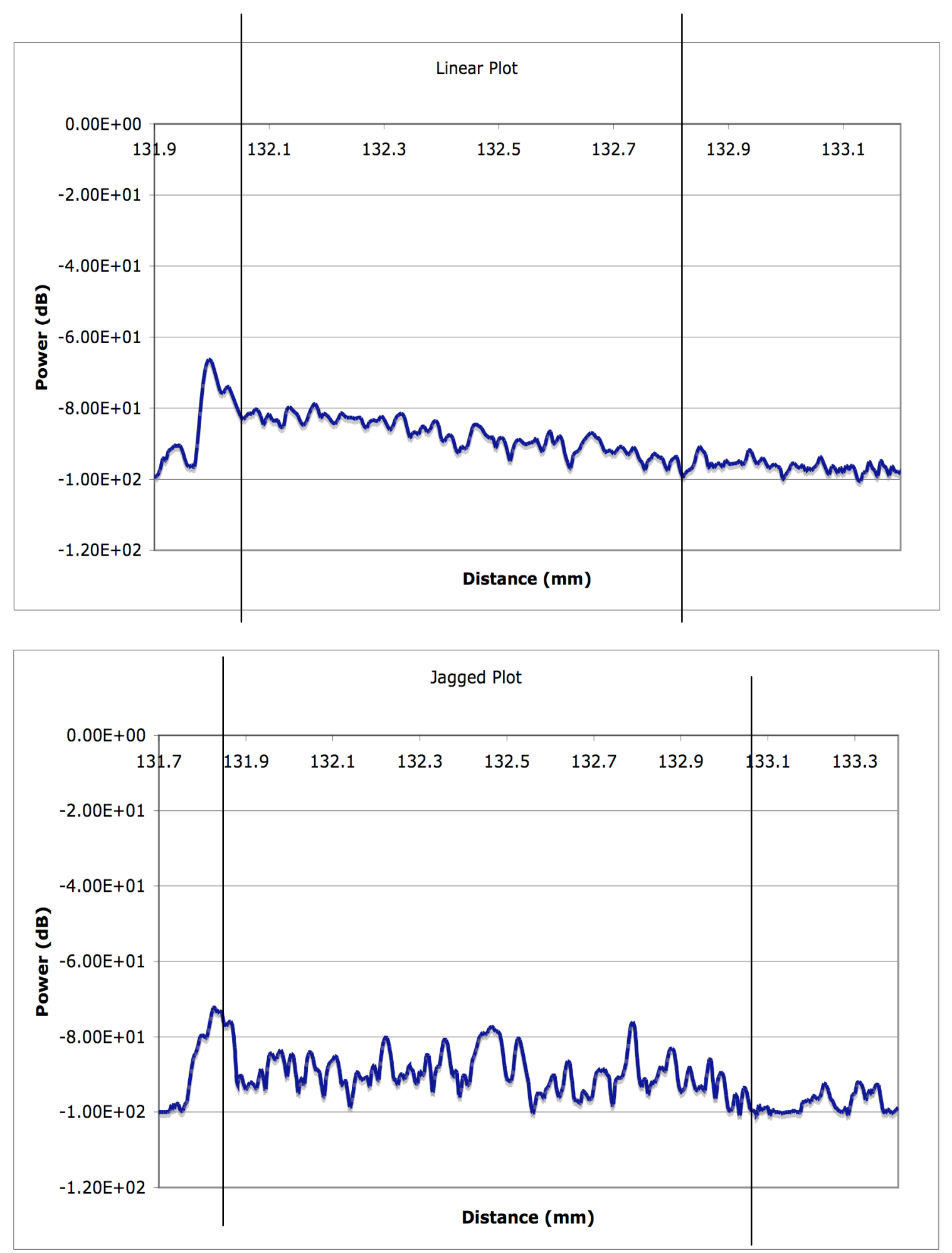

Figure 2.15: The first figure shows a relatively linear plot with $\mathrm{R}^{2}=0.8$. The second one shows a more jagged plot with $\mathrm{R}^{2}=0.12$. The data used for finding the $\mathrm{R}^{2}$ values was limited to what is contained between the vertical lines. 
For the next comparison, it was considered that maybe not all of the samples were usable. Samples that gave lines with low $\mathrm{R}^{2}$ values were eliminated, and only ones with $\mathrm{R}^{2}$ of at least 0.8 were included. The slopes for these samples was then compared with WBS values. The plot for this is shown in Figure 2.16. Even when only considering the samples that had a fairly smooth decay in reflected power, there is no meaningful pattern in the data.

The last comparison made was between penetration depth and WBS. The reason for this comparison is that the WBS value for each piece of meat is known to be a meaningful value that indicates how tender the meat is, and a difference in tenderness could affect how deep light is able to penetrate into the meat. The data is shown in Figure 2.17. Based on this plot, there does not appear to be any relationship between penetration depth and WBS value.

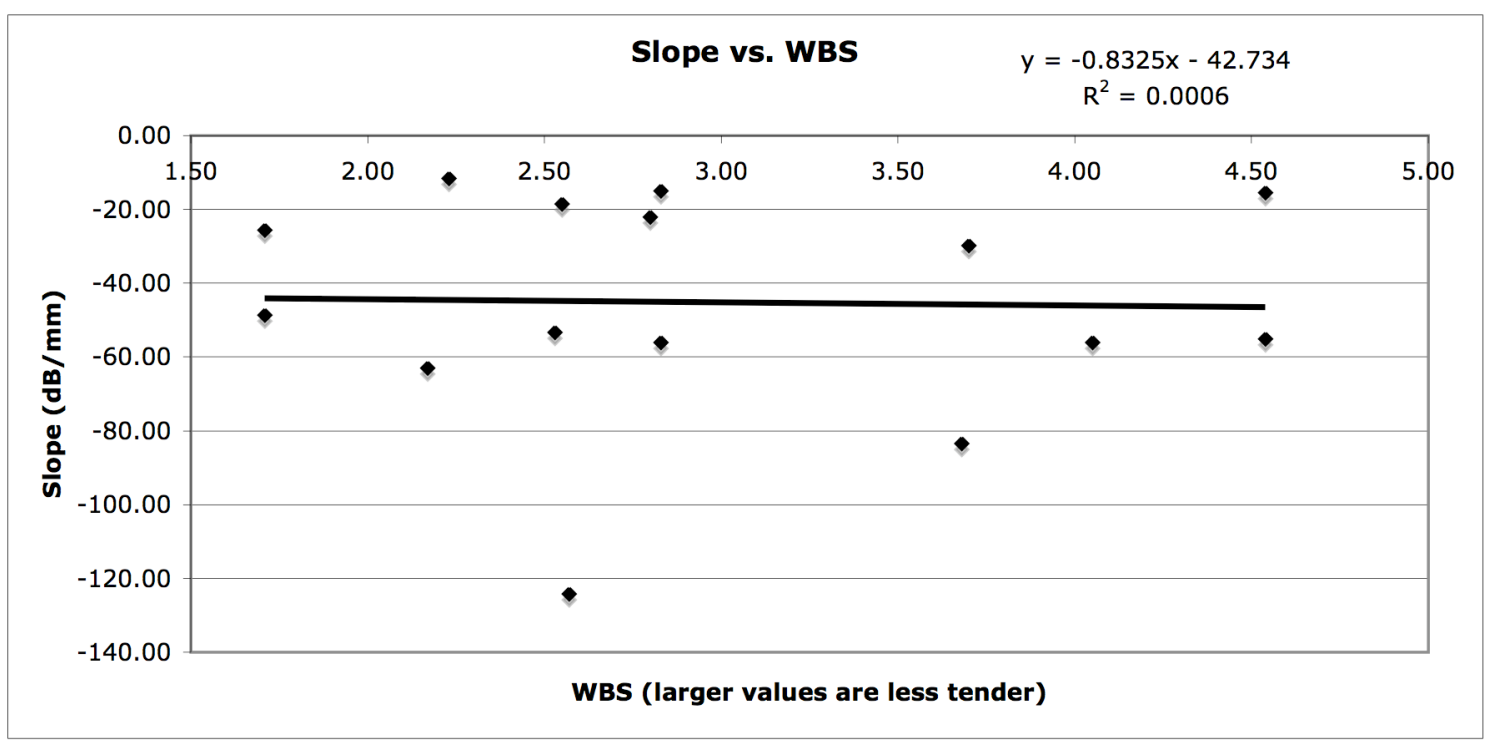

Figure 2.16: Comparison of the slopes of OCT measurements on meat samples with the WBS values for those meat samples, only considering samples that have fairly linear power-vs.-distance plots $\left(\mathrm{R}^{2}>0.8\right)$. The shallow slope and small $\mathrm{R}^{2}$ value indicate that there is no correlation between these values. 


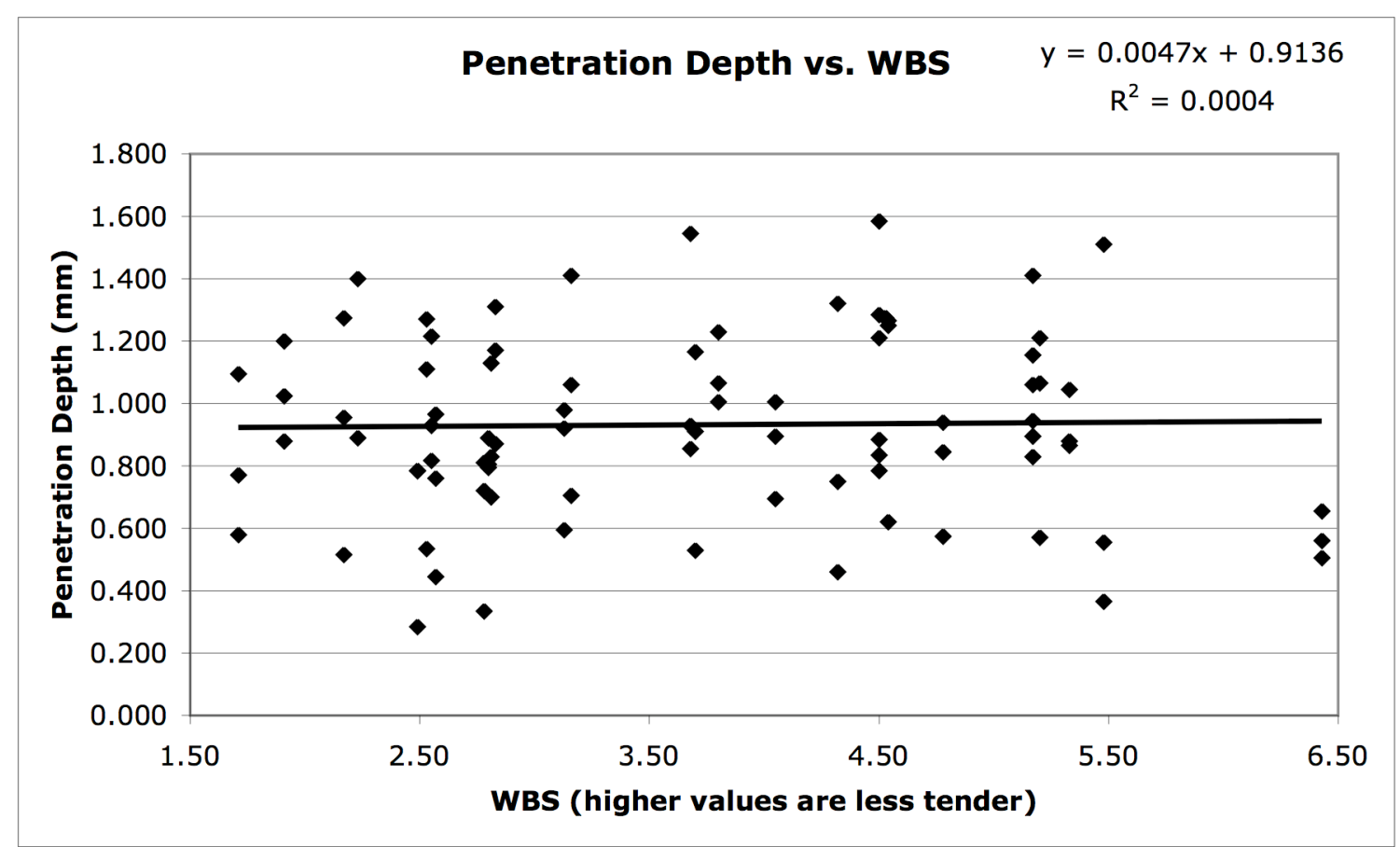

Figure 2.17: Examining the relationship between penetration depth of the OCT measurement and WBS values for the samples. The shallow slope and small $\mathrm{R}^{2}$ value indicate that there is no correlation between these values.

\subsection{Conclusions}

No correlation was found between WBS values for meat tenderness and the OCT

signature. The average penetration distance of the light into the meat was about $0.9 \mathrm{~mm}$, measured using the criterion of at least $-95 \mathrm{~dB}$ power reflecting back from that depth. This means that, if any meat tenderness conclusions can be made from this type of measurement, it would depend on the surface of the meat being representative of the entire meat sample. No effort was made to determine specifically if there was a correlation between tenderness of the meat on the surface and tenderness of the entire piece of meat. 
Based on the comparisons done, there was no noticeable connection between the OCT measurements and the meat tenderness values. This conclusion is based on several simple methods of analysis. It is possible that more complex statistical methods would be able to find some connection between the OCT data and meat tenderness.

This setup was fairly slow, with a measurement repetition speed of approximately $1 \mathrm{~Hz}$. Since about 60 measurement sweeps had to be averaged together to get a good sample, each measurement took over a minute. A major factor limiting the speed of this measurement is the fact that it relies on a movable mirror. There is only so much that can be done to speed up the back-and-forth movement of the mirror used in the setup. Due to this limitation, swept-wavelength sources are becoming more popular, and white-light interferometry is being replaced by techniques that take advantage of these sources.

\subsection{Improvements From Other Sources}

The reflectometer that was used is not the fastest that is currently on the market. The fastest source on the market is the Rapidly Swept Tunable Laser from Thorlabs [10]. This laser sweeps through its wavelength range at a $16 \mathrm{kHz}$ repetition rate. The primary goal for the rest of this paper is to show that the SGDBR laser can potentially be used to make measurements more quickly than this product. 
CHAPTER 3: Chip-level Investigation of the SGDBR Laser

The next part of this paper will discuss the work to demonstrate that SGDBR lasers can be used for high-frequency wavelength switching. The discussion will first cover the work with a chip laser and explain those results. This will be followed with a discussion of work done with a packaged laser.

\subsection{Description of the SGDBR Chip Laser}

One of the chip lasers that were provided by UC Santa Barbara (UCSB) can be seen in Figure 3.1. These lasers were chosen because they had the potential to be used for very fast wavelength switching. The AlN substrate is approximately $5 \mathrm{~mm} \times 7 \mathrm{~mm}$, and the laser itself is approximately $0.5 \mathrm{~mm} \times 3 \mathrm{~mm}$.

The substrate serves several purposes. First, it has thermal properties that allow it to match the expansion of the laser. It is also good for conducting heat away from the laser and acting as a heat sink. The laser is mounted on the substrate using gold-tin solder to improve the effectiveness of the substrate as a heat sink.

There is an SOA, a front mirror, two gain sections, phase, and a back mirror. More information about SGDBR lasers can be found in section 1.2 of this paper. Leif Johansson at UCSB provided rough values for "safe" current limits, which are listed in Table 3.1. 


\begin{tabular}{|l|r|}
\hline Laser Element & Maximum Current (mA) \\
\hline SOA & 120 \\
\hline Gain & 120 \\
\hline Phase & 15 \\
\hline Front Mirror & 20 \\
\hline Back Mirror & 40 \\
\hline
\end{tabular}

Table 3.1: Maximum safe current values for the different laser components.

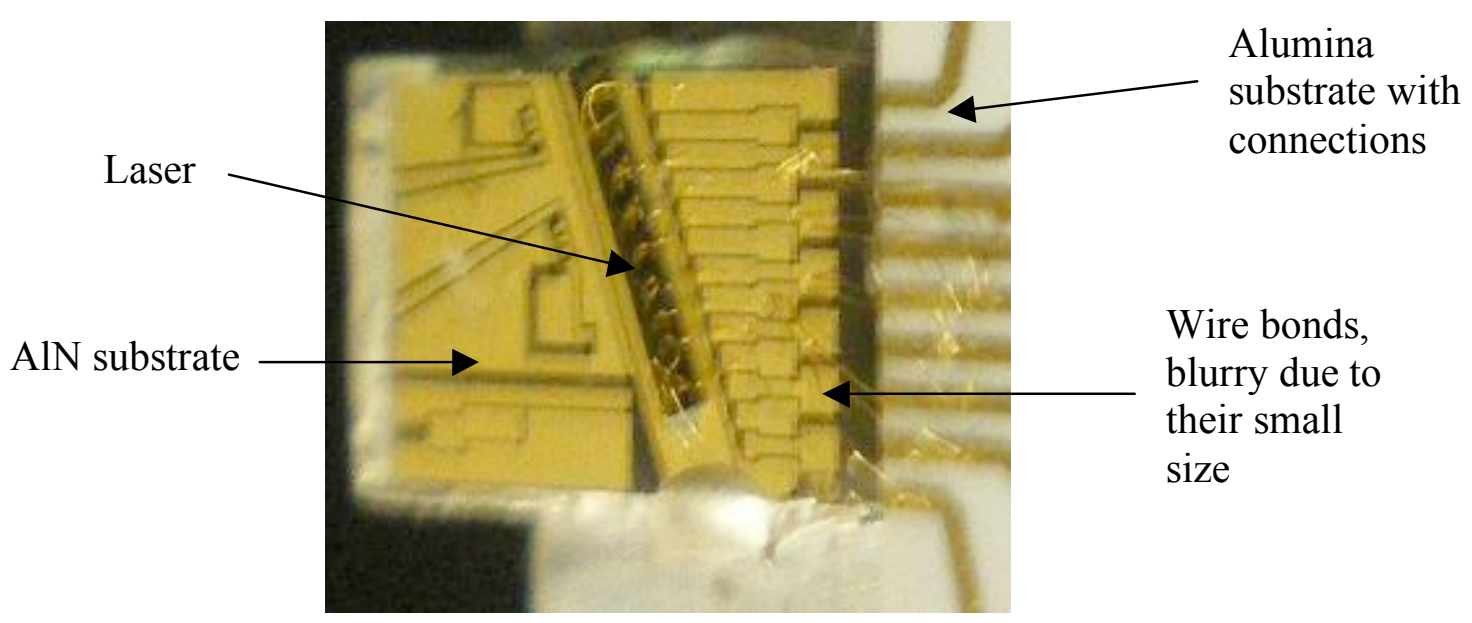

Figure 3.1: A close-up picture of the chip laser used.

\subsection{Package Construction}

Figure 3.1 shows the laser along with the base that it was manufactured on. There are 13 pads to use for making connections with on one side of the base. This picture also shows the circuit board and wire bonds used to connect to the laser, which are described next.

Figure 3.2 shows the base used for holding the laser and providing current. The starting pieces were four gold-plated copper bases with Wiltron K connectors attached to them. Mike Bernacil made thin film circuit boards and used silver-loaded conductive epoxy (EpoTek H20) to attach those onto the copper bases. Aluminum bases were created to put the actual laser on, and those were attached to the copper bases. 
The rest of the package construction had to be done at UCSB. The first thing to do was put the laser onto the aluminum base. The first step was to apply a thin, smooth layer of silver epoxy to the aluminum base. Then tweezers were used to carefully put the laser on the epoxy and line it up. The silver epoxy layer between the base and the laser's substrate allowed for a good thermal conduction. At this point epoxy was applied to connect between the aluminum base and the laser base to provide for a good ground connection.

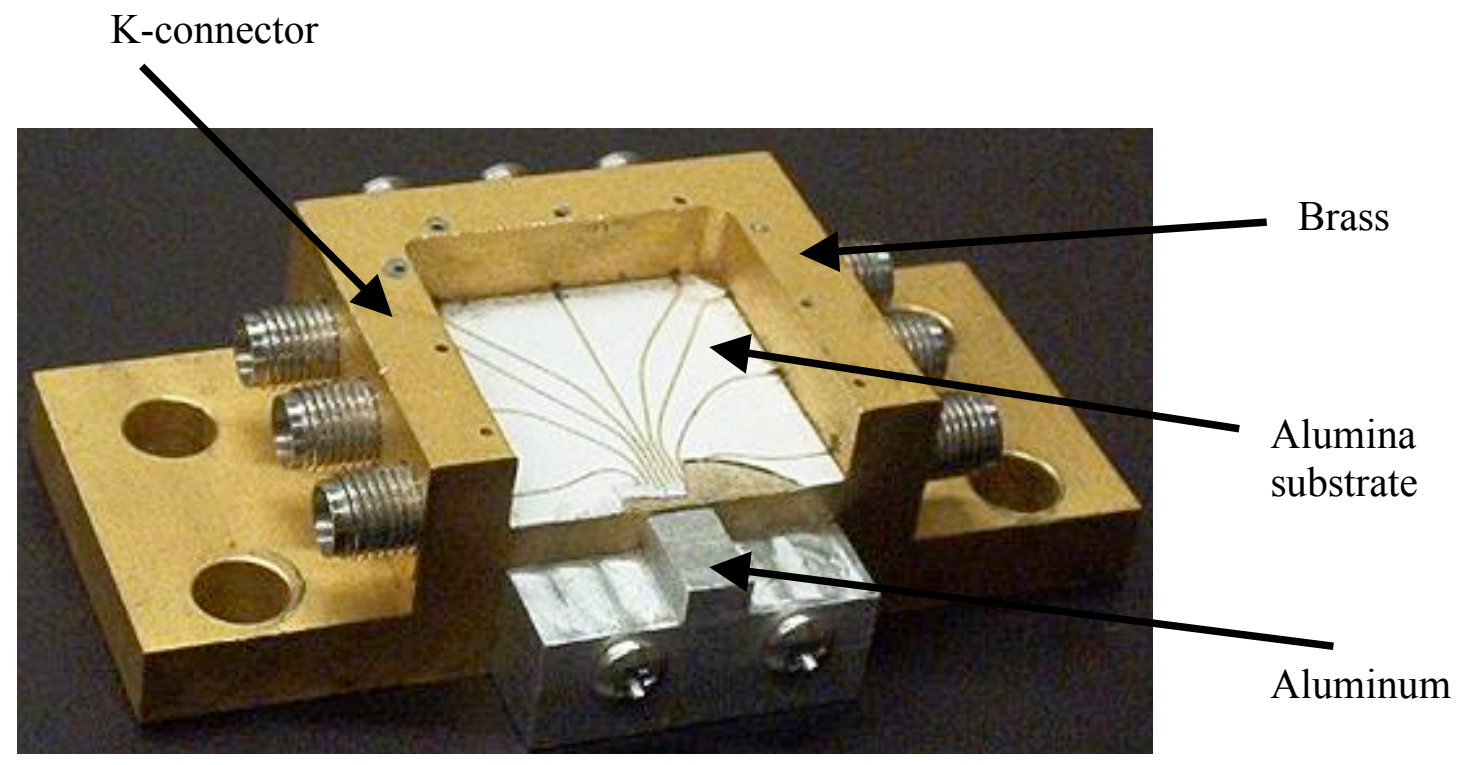

Figure 3.2: The base used for the laser. The aluminum base was attached to the front, and the brass portion has $\mathrm{K}$ connectors all around it. This particular one had a piece of the alumina substrate chipped off; the others did not have this problem. 


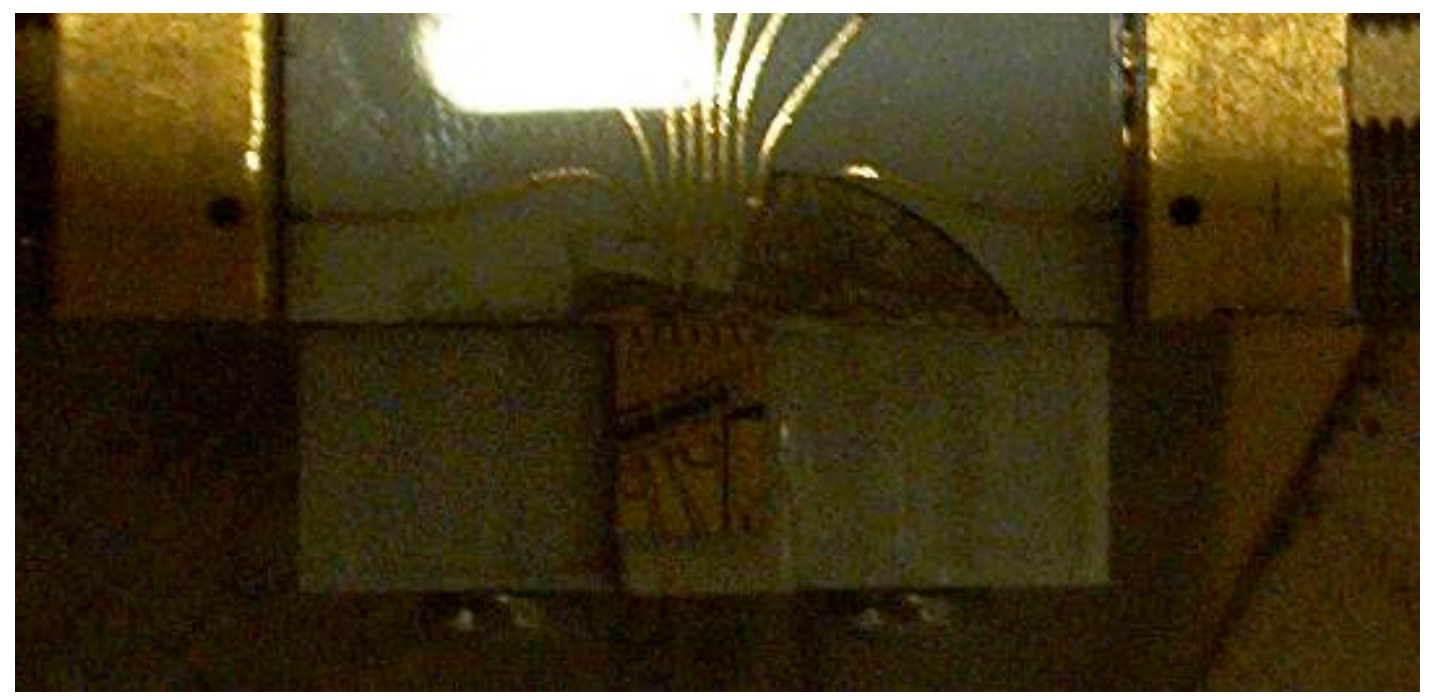

Figure 3.3: The laser being mounted onto the base, with the laser at the bottom of the image

Figure 3.3 shows the laser being attached to the base. Around the actual laser there are several spots to make connections, and the most convenient ones are placed closest to the alumina substrate. The laser itself has also been positioned at the edge of the raised portion of the laser mount so that it is as accessible as possible later on. With the laser put in place, the whole thing had to be heated to harden the epoxy. The laser package was put onto a hot plate at $120^{\circ} \mathrm{C}$ for about 90 minutes.

The last part of the package construction was to create wire bonds to connect the laser to the alumina substrate. For each connection, a wire bond had to be created between the laser and the base that it was built on, and another wire bond had to be made between the base and the circuit board. Because of the delicate nature of these connections and the lasers, Leif created the wire bonds. A picture of this is shown in Figure 3.4. 


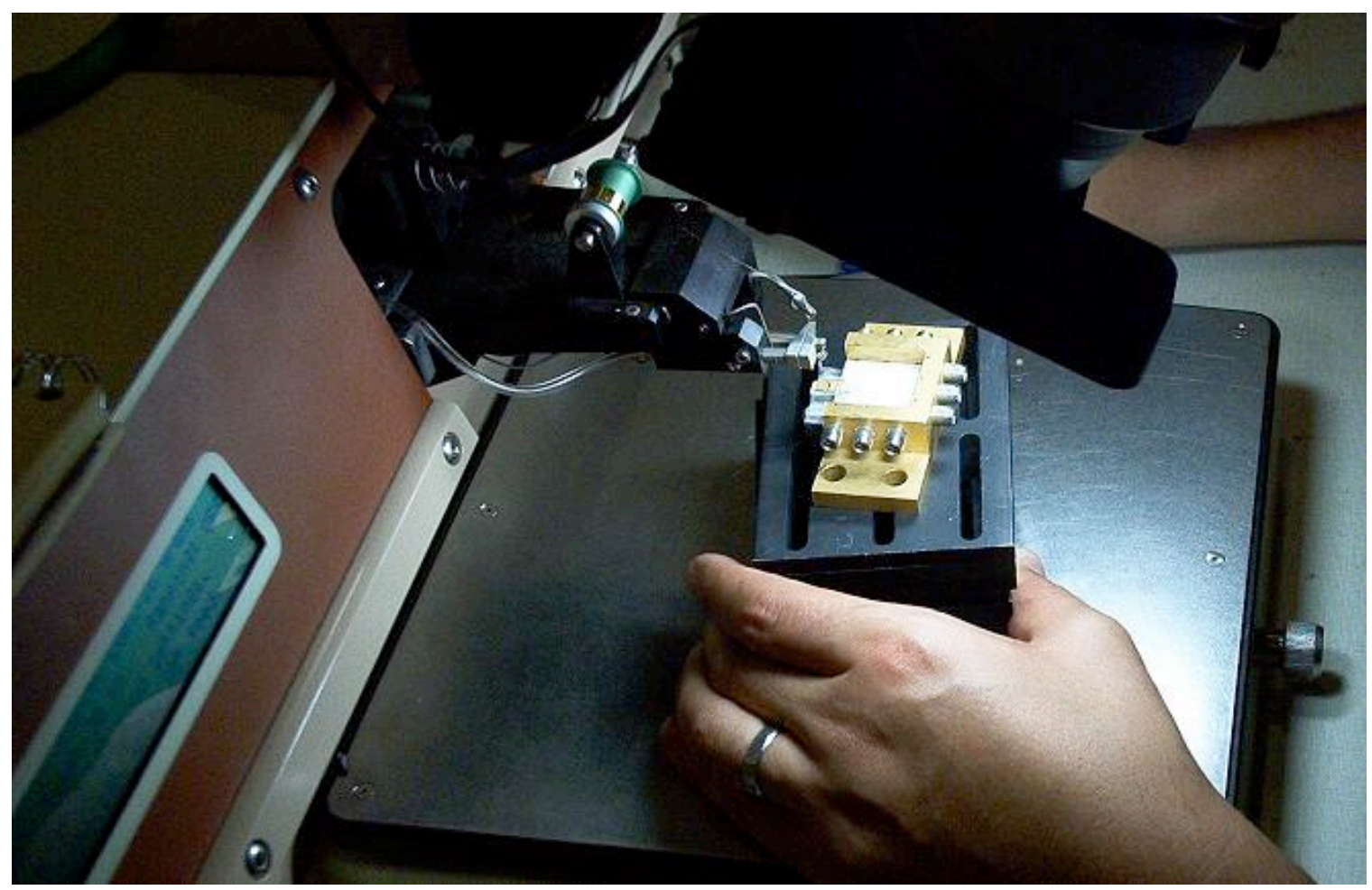

Figure 3.4: Wire bond connections being created for the laser

\subsection{Verifying That the Lasers Work}

The first thing done with the lasers was verifying that they worked. They were tested by applying currents to the front mirror, back mirror, and gain sections, and then applying a reverse-biased voltage to the SOA section. By reverse-biasing the SOA, it acts as a photodetector instead of an amplifier. The light coming from the rest of the laser creates a current in the SOA that can be measured, verifying that the laser is operating as expected.

Figure 3.5 shows the setup used. $\mathrm{I}_{\text {out }}$ is sent into the SOA element. The resistor provides current limiting to protect the laser. The voltage is read off of the voltage source and across the resistor; the difference between these voltages is the voltage across the SOA, and that voltage difference is recorded. Table 3.2 shows the measurements from this setup. 


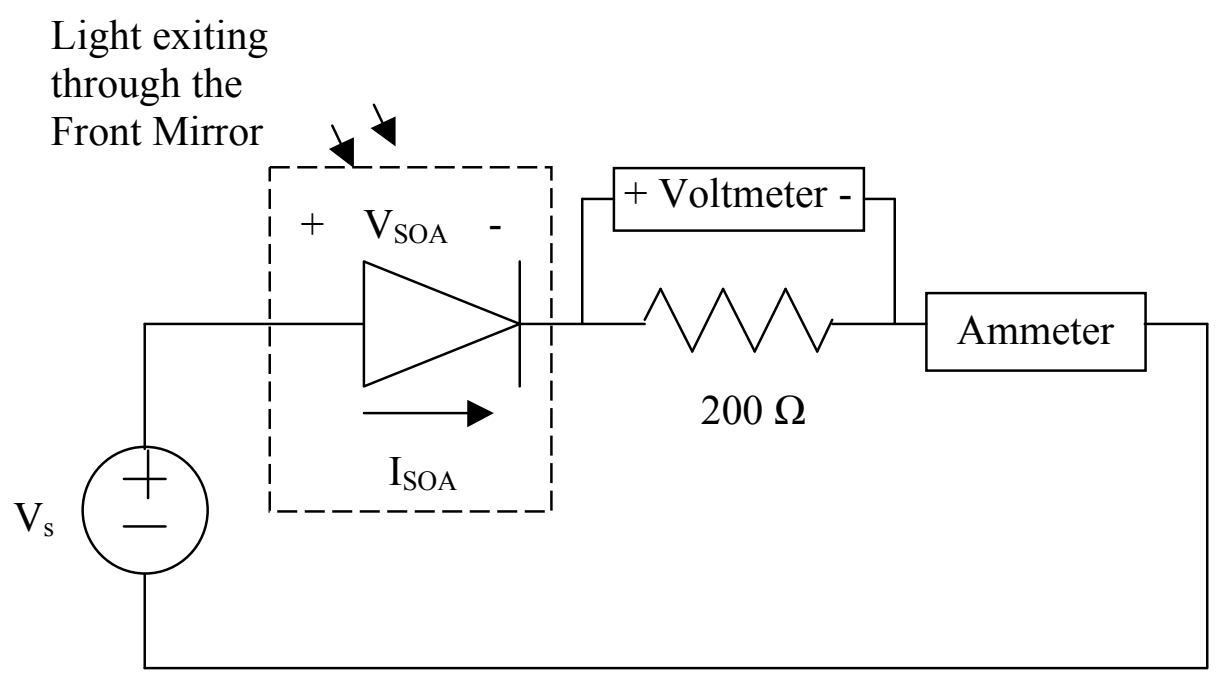

Figure 3.5: Schematic for checking that the lasers work. DC bias conditions: $I_{\text {gain }}$ $=80.0 \mathrm{~mA}, \mathrm{I}_{\mathrm{FM}}=\mathrm{I}_{\mathrm{BM}}=20.0 \mathrm{~mA}, \mathrm{I}_{\text {phase }}=0 \mathrm{~mA}$.

With two lasers verified to be functional, the next step was to verify that light was actually coming out of the laser, which was done using a power meter. Holding the power meter in front of the laser gave a reading of $-36 \mathrm{dBm}$. Blocking the path between the laser and the power meter dropped the reading to $-51 \mathrm{dBm}$ (due to the light in the room). In addition to that, varying the SOA current caused a corresponding change in the power reading, confirming that two of the lasers were functional.

\begin{tabular}{|c|c|c|c|c|}
\hline \multicolumn{5}{|c|}{ Laser C } \\
\hline $\mathrm{I}_{\mathrm{FM}}(\mathrm{mA})$ & $I_{\mathrm{BM}}(\mathrm{mA})$ & $\mathrm{V}_{\text {SOA }}(\mathrm{V})$ & $\mathbf{I}_{\text {Gain }}(\mathbf{m A})$ & $\mathrm{I}_{\mathrm{SOA}}(\mathrm{mA})$ \\
\hline \multirow{3}{*}{20.0} & \multirow{3}{*}{20.0} & -2.01 & 80.0 & -3.874 \\
\hline & & -1.00 & 80.0 & -3.724 \\
\hline & & -2.00 & 70.0 & -1.673 \\
\hline \multicolumn{5}{|c|}{ Laser D } \\
\hline 9.3 & 21.7 & -2.00 & 80.0 & $\begin{array}{r}\text { approx. }-3 \mathrm{~mA} \\
\text { (exact value not } \\
\text { recorded) }\end{array}$ \\
\hline \multicolumn{5}{|c|}{ Laser $A$ and $B$} \\
\hline & & & 0.0 & \\
\hline
\end{tabular}

Table 3.2: Data showing the functionality of the lasers. Laser $\mathrm{C}$ and Laser $\mathrm{D}$ are operational. Laser A and B have bad connections to the Gain element. 


\subsection{Bench Setup}

Once the laser packages were put together, the next step was to hook them up and test how they worked. Figure 3.6 shows several things about the setup. First, the whole setup was put on a 12 in $\mathrm{x} 12 \mathrm{in}$ black aluminum breadboard, which made it portable. This was secured to a larger table during actual use. The lens setup was attached to an XYZ stage, so an aluminum base was used to raise the laser up. Each laser input has its own current source, which is connected to the laser through a BNC cable and a BNC to Kconnector adapter. Figure 3.7 shows the position of the lens setup compared to the position of the laser.

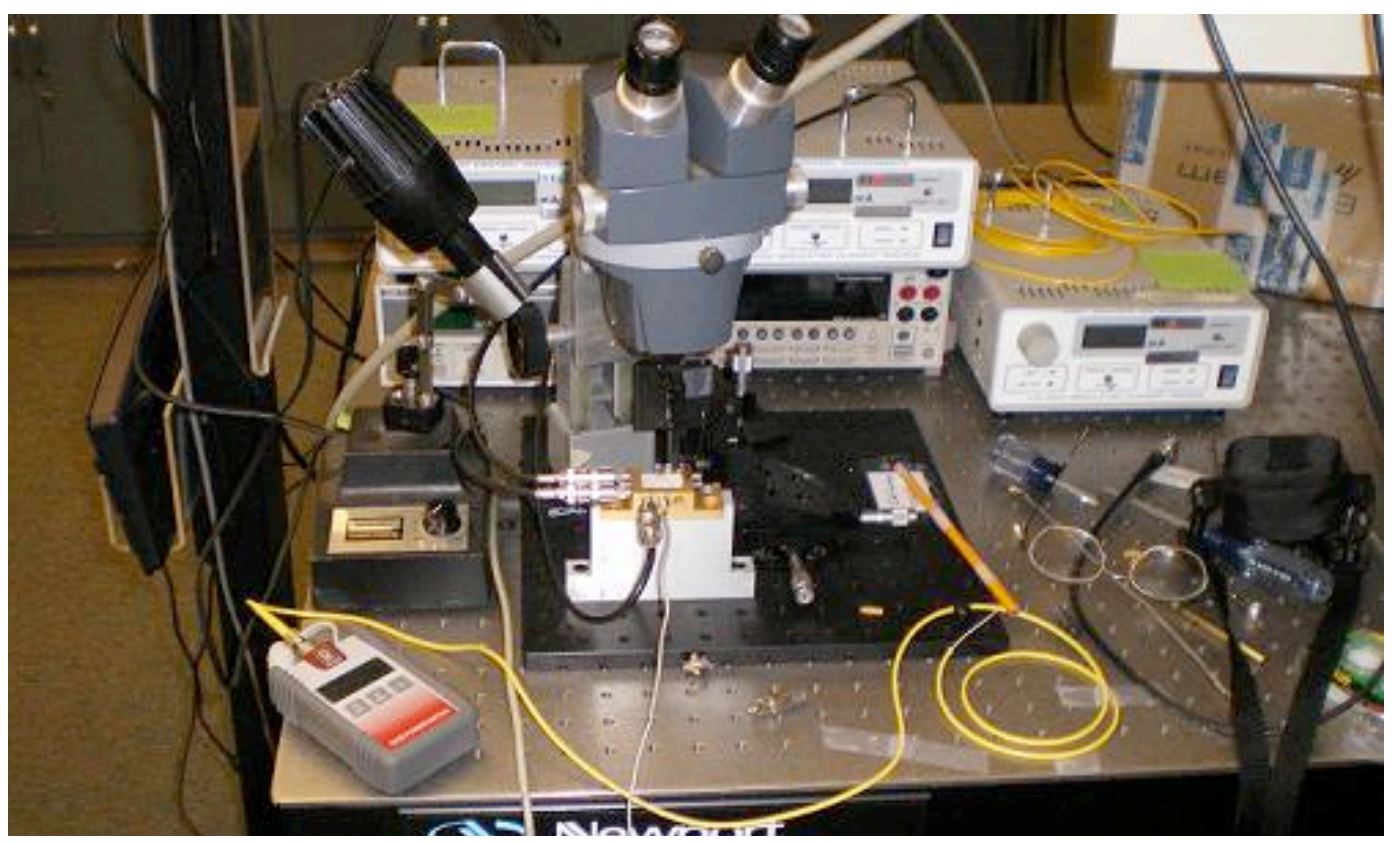

Figure 3.6: The bench setup for the laser 


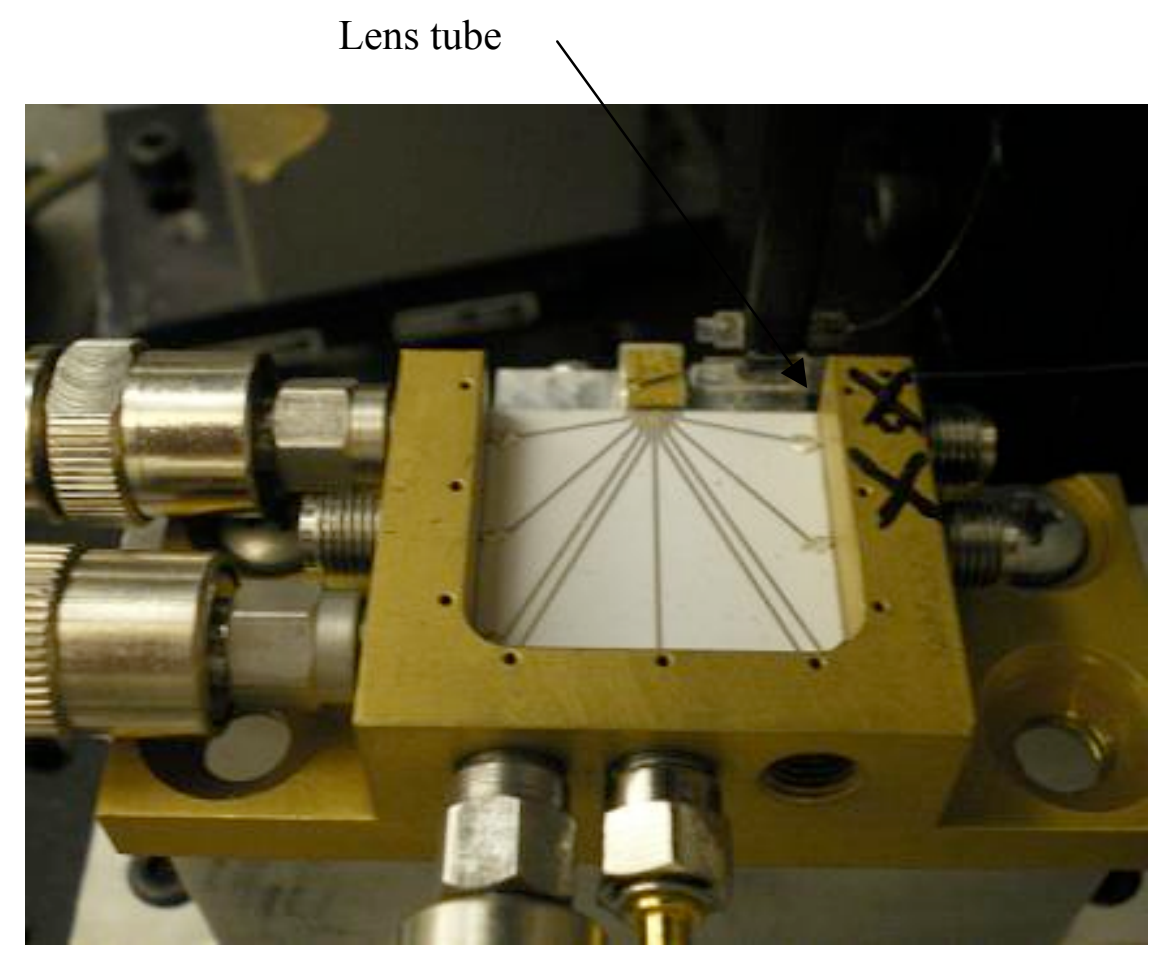

Figure 3.7: Close-up picture of the lens setup positioned right next to the laser. For reference, the outer diameter of the tube holding the lens is $2.8 \mathrm{~mm}$.

\subsection{Optical Setup}

There were a few choices to make when picking a lens setup for coupling light. The first decision made was to use a GRIN lens to focus light on a pigtailed ferrule. The lenses are made for either $1300 \mathrm{~nm}$ or $1560 \mathrm{~nm}$ light; the laser is around $1560 \mathrm{~nm}$, so lenses designed for that were used. For the GRIN lens, 0.23 pitch and 0.29 pitch lenses were both available to pick from. Other choices to make were whether to use angled edges or flat edges for the lens sides facing either, and whether to use a flat or curved face for the front of the GRIN lens. Several configurations were tried.

\subsubsection{Lens Pitch}

One of the early decisions made was the pitch to use for the GRIN lens. To understand this decision, first the structure of a GRIN lens must be explained. The basic 
principle in a GRIN lens, as in all lenses, is Snell's Law, which says that light will change direction whenever it moves into a medium with a different index of refraction than the medium it just left. With a typical "thin" lens, all of the focusing is done by refraction at the surfaces of the lens. With a GRIN lens, the refraction also occurs within the lens. Inside of a GRIN lens, the index of refraction decreases as the radius increases. This results in light following a sinusoidal path through the lens, regardless of how it enters the lens. This is shown in Figure 3.8. A lens that is 1.00 pitch would have light coming out in exactly the same direction that it entered in, almost as if it had not gone through a lens at all (besides the fact that it has been shifted in the direction of the GRIN lens axis). In general, the pitch indicates the portion of a full sinusoid period that light travels through inside of the lens. The pitch for a lens is proportional to its length. 

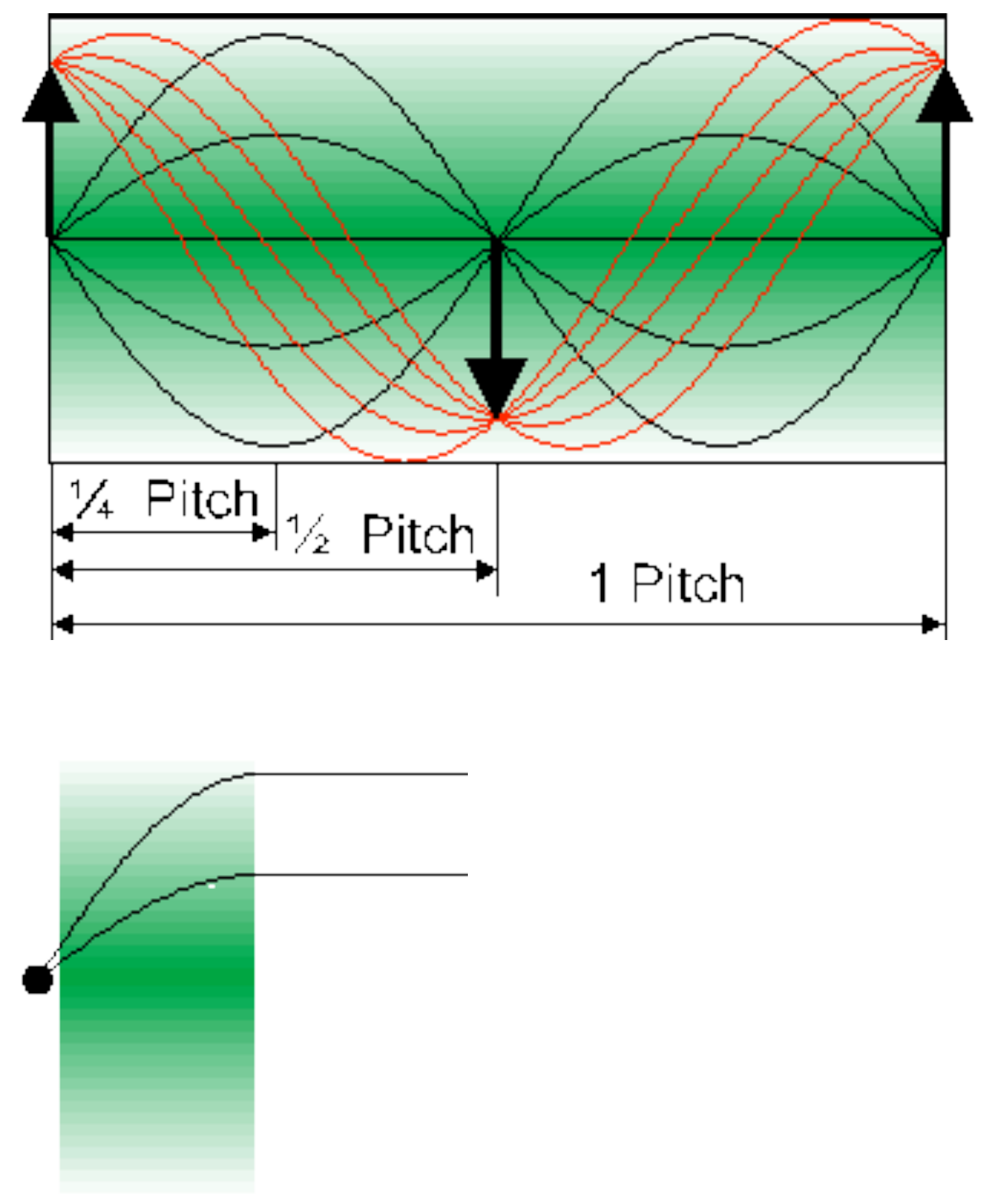

Figure 3.8: GRIN lens diagram. The top picture shows how a GRIN lens works. A 1 pitch lens has all light go through a full sinusoidal cycle. The bottom picture shows the planned setup. In this setup, the light goes through a 0.23 pitch GRIN lens which causes it to come out parallel to the GRIN lens axis [5].

For this setup, the goal is to collimate the light (have it leave the lens such that all of the light is traveling parallel to the lens axis) as shown in Figure 3.8. The exit point on the laser is small enough to be treated like a single point (relative to the size of the GRIN lens), and it is highly divergent. If the lens could be directly next to the source, then a 0.25 pitch lens would work. Instead of doing that, the lens is set up a small distance 
away from the laser, which means that the light enters the lens at a later part of the sinusoidal path. Therefore, the lens needs to be slightly less than 0.25 pitch. Since the available options were 0.23 pitch and 0.29 pitch, a 0.23 pitch lens was used.

\subsubsection{Lens Configuration Testing}

The first setup used a flat face for the GRIN lens and angled edges on the sides facing each other. However, there was no precision involved in their placement, and the angled sides were not lined up relative to each other. The GRIN lens was epoxied in place, and the ferrule lens wouldn't move, so it could not be adjusted. The output from the laser was measured to be approximately $-60 \mathrm{dBm}$. Since the output was expected to be around $0 \mathrm{dBm}$, or possibly as low as $-10 \mathrm{dBm}$ due to inefficiencies in the lens setup, a poor lens configuration was thought to be the cause.

For the next setup the same lenses were used. This time, the NSG America web site was used to calculate the distances that should be used for the setup [8]. In addition, the angled sides ( $\left(8^{\circ}\right.$ angle) were lined up so that they were parallel. This setup did not provide a meaningful improvement in the power detected at the output. In fact, it added another strange effect - the lens setup needed to be aligned to the side of the end of the laser to maximize the captured light power. This strange alignment is shown in Figure 3.9. 


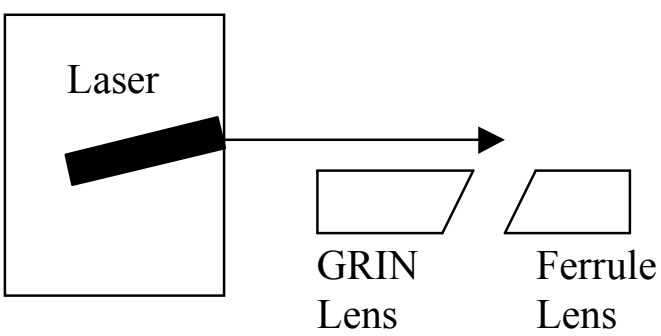

Figure 3.9: Lens setup aligned to the side of the laser. The arrow indicates the direction of maximum light output from the laser. To achieve maximum power at the photodetector, the lens was lined up away from the path of highest light output

For the third setup, shown in Figure 3.10, a plano-convex GRIN lens and a flat ferrule lens were used. This meant that there would be nothing to cause problems with light transmission, but it came at the cost of potentially letting reflected light go back to the laser. When this setup was aligned for maximum power, the lens was straight in front of the laser, which indicated that the angled edges were causing the misalignment seen in the previous setup. Unfortunately, this did not fix the problem of low power readings either.

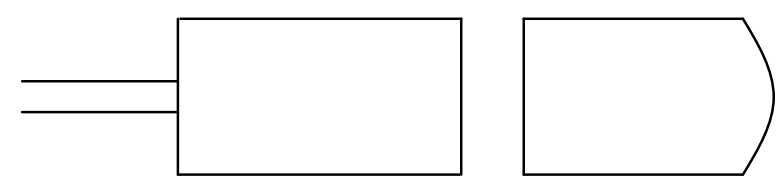

Figure 3.10: Third lens setup used. Lens setup with a convex front on the GRIN lens and flat edges on the internal surfaces

Due to the unsatisfactory measurements from the previous setups, the next step was to make sure the lens setup worked properly. To do this, a distributed feedback (DFB) laser was used. The first thing checked is that the beam could be collimated using the GRIN lens; this was done without any issues. The next step was to determine if light was being received by the ferrule lens and transmitted through the fiber properly. Using 
the lens setup from Figure 3.10 with the laser-to-lens distance being $0.75 \mathrm{~mm}$, the power that came out of the fiber was about $-2 \mathrm{dBm}$. The power coming directly from the laser was about $4 \mathrm{dBm}$. This meant that the lens setup was causing a $6 \mathrm{~dB}$ drop. Since the measurements from the setup with the SGDBR laser were roughly $50 \mathrm{~dB}$ lower than expected, the lens setup and fiber were not the problem.

\subsubsection{Visual Observations of Laser Output}

One of the final observations with this setup came from using an infrared (IR) card in front of the laser. When the room lighting was reduced sufficiently it became possible to see the laser light on the IR card. This task was complicated by the fact that the IR card had to be very close to the laser for light to be visible, but at the same time it couldn't touch the laser because that would likely damage the laser. At one distance there was a nice, relatively bright spot. When the IR card was moved a little bit further away, there were two visible spots, on either side of where the main one had been. It was difficult to tell, but there may have been four spots instead of just two. The expected result was a single spot at all distances, with one distance having a small focused spot and other distances having a larger, fainter spot.

This observation led to the conclusion that there were multiple modes of light coming out of the laser, and this was the reason that light couldn't be transmitted through the lens. There was a TEM $\mathrm{TO}_{00}$ mode, which is just a single spot, and there was also a $\mathrm{TEM}_{01}$ mode, which is two spots to either side of the central beam path. These are shown in Figure 3.11. The interference of these two modes is the probably the reason that light couldn't be focused into the lens setup. 

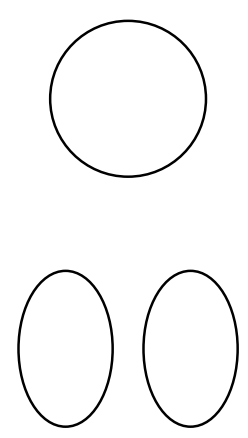

Figure 3.11: Light modes from the laser. The top shows the $\mathrm{TEM}_{00}$ mode, and the bottom shows the $\mathrm{TEM}_{01}$ mode.

\subsection{Wavelength Tuning Efficiency Measurements}

Although there was poor coupling efficiency of the light into the fiber, some light could still be measured at the end of the fiber. The light that made it into the ferrule lens and into the fiber was fed into the OSA. The phase current was varied, and the output wavelength and power were measured. Figure 3.12 has a plot showing the linear relationship between the phase current and the output wavelength. See Appendix $C$ for a table with the data. 


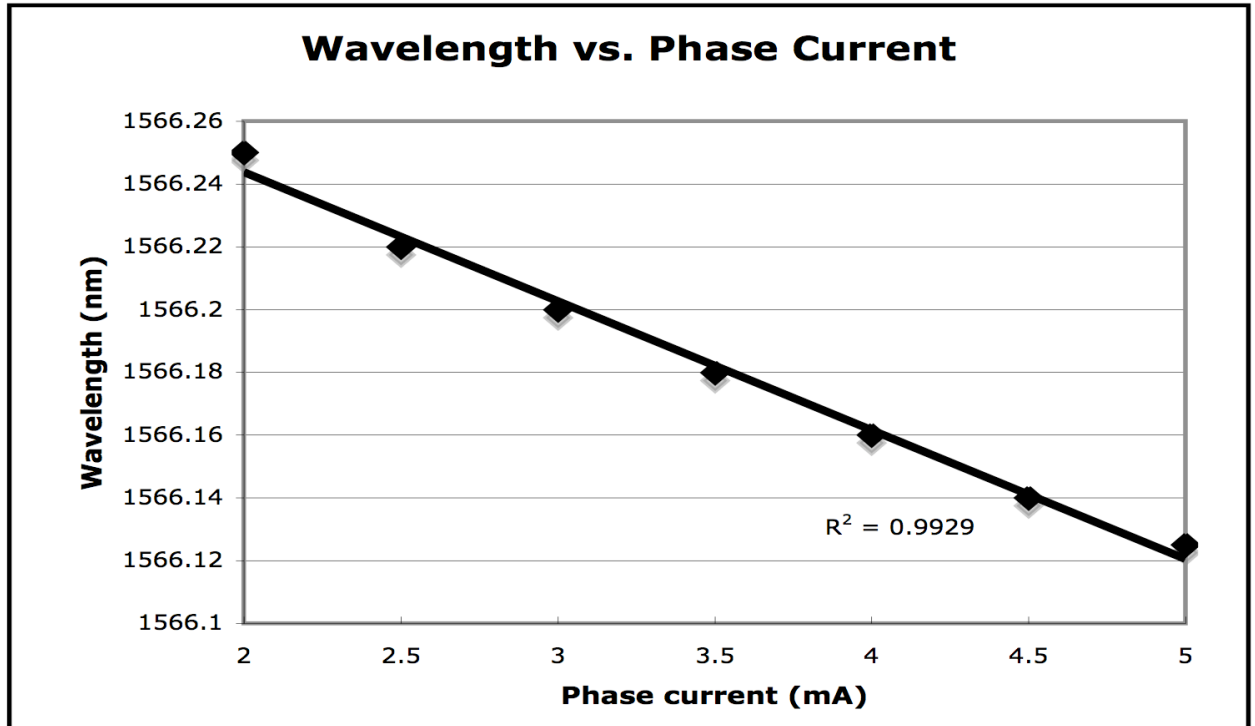

Figure 3.12: Wavelength versus phase current for the chip laser, showing a very linear relationship. $\mathrm{I}_{\mathrm{FM}}=14.8 \mathrm{~mA}, \mathrm{I}_{\mathrm{BM}}=31.0 \mathrm{~mA}, \mathrm{I}_{\text {gain }}=81.6 \mathrm{~mA}, \mathrm{I}_{\mathrm{SOA}}=$ $80.5 \mathrm{~mA}$, OSA Resolution BW $=0.2 \mathrm{~nm}$, Video BW $=194 \mathrm{~Hz}$

\subsection{Next Steps for Using Chip Lasers}

To improve on this setup, the lasers would need to be manufactured for this purpose such that the light can be focused into a lens system. The functionality of the lens system was verified using another laser. A reasonable amount of light was coming out of the laser; this was verified both by using an IR card to visually inspect the laser output and by using a power meter to measure the light coming out. The tuning ability of these lasers was also verified. If the light could have been focused into the lens system, then this system would most likely have been successful. Since this system didn't work well enough, another approach was explored. 


\section{CHAPTER 4: High Speed Packaged SGDBR Lasers}

\subsection{Custom-Packaged SGDBR Lasers}

The next step setup used pre-packaged lasers provided by JDSU. The laser packages they traditionally create are designed for the telecommunications industry. This is important to note, because the normal use of these lasers is to operate them at a single frequency. Initially, the industry used DFB lasers, which are manufactured to operate at a single wavelength and cannot be tuned to any other wavelengths. There were multiple lasers to maintain, one for each frequency used. In addition to having a laser in operation at each wavelength, a backup laser was needed for each wavelength in case one stopped working.

SGDBR lasers were used as a different method for keeping backup lasers. Instead of having a backup laser for each wavelength, a single SGDBR laser could be tuned to whichever wavelength was needed and put into use. This saved on excess inventory that companies had to maintain. The laser that JDSU provided was initially designed for this type of telecommunications use.

\subsection{Goal}

The goal of this work is to create a continuous wavelength ramp from the lowest to highest frequency in as short a period as possible. Previous work on high-speed wavelength switching showed the ability of the SGDBR laser to switch wavelengths in less than $5 \mathrm{~ns}$ [9]. The group that achieved this did several things to reach that point. The sampled grating mirrors had reduced peak spacing, reducing the current change needed to 
tune the front and back mirrors. They also built the tuning sections to improve the heat sink efficiency. The other thing they did is apply a pre-emphasis to the input pulses to cause the wavelength to settle down more quickly, as shown in Figure 4.1. These steps are not done with this work, but other efforts are made to improve the switching speed.
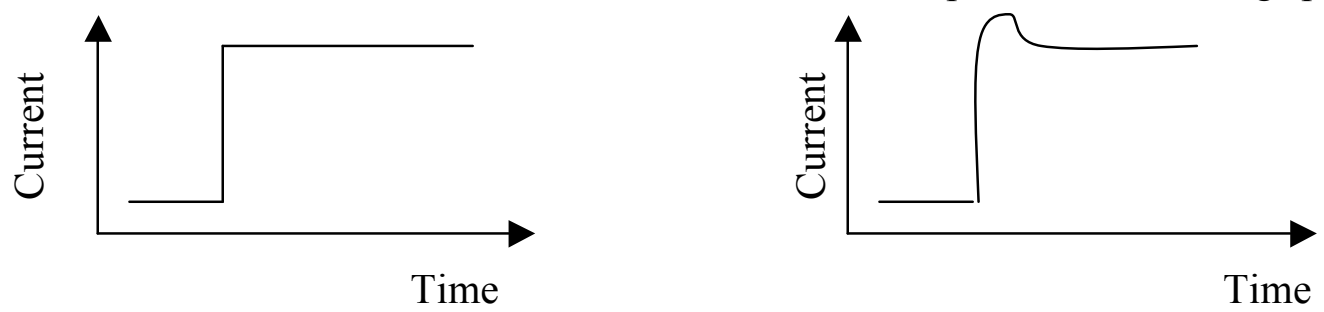

Figure 4.1: Demonstration of pre-emphasis. The signal on the left is an example plot of current versus time, "instantly" jumping from the starting value to the ending value. The signal on the right intentionally overshoots the ending value to cause the laser to settle at the desired current and its associated wavelength more quickly; this process is pre-emphasis.

\subsubsection{Obstacles}

There are several obstacles limiting how quickly the wavelength can be varied. The laser used is the MKS-063-B, made by JDSU. As mentioned above, the laser was made for the telecommunications industry. The MKS-063-B laser inputs had incorporated low-pass filters to prevent small, rapid input changes from affecting the laser's output wavelength. JDSU removed the filters in the lasers they provided Cal Poly. There are other obstacles also. The butterfly leads used to connect to the interior of the laser are a source of inductance. Since these leads are outside of the laser package, the inductance can be reduced by adding capacitance to compensate. The segments within the laser have some capacitance to them, which can be characterized but not reduced.

\subsection{Constructing the Laser Driver Circuitry}

The datasheet showing the laser layout can be found in Appendix D. By removing the low-pass filters, JDSU eliminated the biggest obstacle to using these lasers 
for high-speed switching. The part that had to be built was the connection between the BNC cable and the butterfly leads coming out from the laser packaging. A 0.085 " semirigid coax cable was broken open and the center conductor was connected directly to the butterfly lead. The end of the semi-rigid coax cables have SMA connectors. An adapter was used to go from the SMA connector to the BNC connector on the cables coming from the current sources.

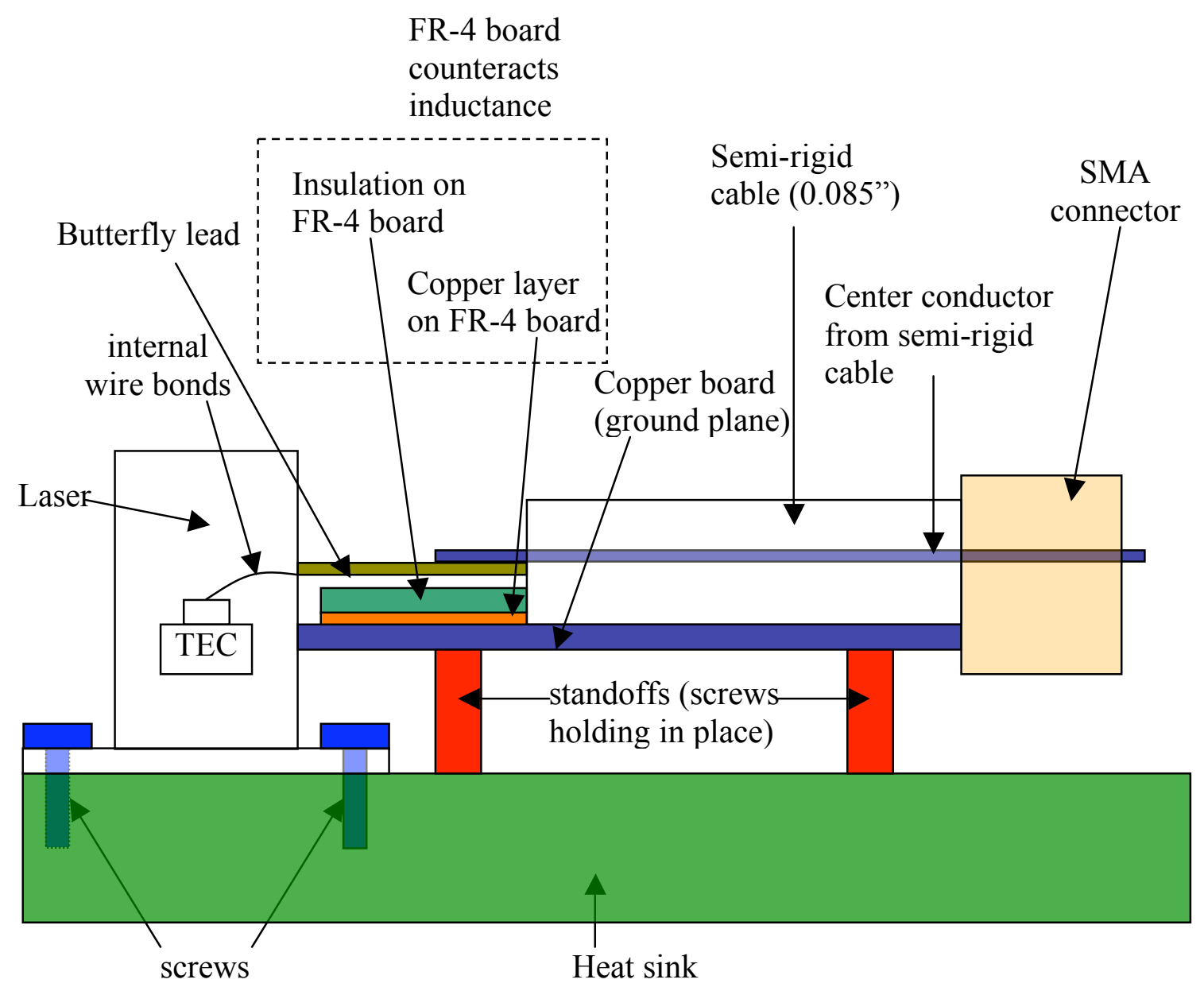

Figure 4.2: Side view of the connection between a semi-rigid cable and a butterfly lead 
To create an effective connection to the laser inputs, several things had to be taken into account. First, the distance between the insulated portion of the semi-rigid cable and the enclosed portion of the packaging needed to be as small as possible to reduce the effects of parasitic inductance associated with the butterfly package leads. The leads from the packaged laser are approximately $10.5 \mathrm{~mm}$ in length. The process of cutting them shorter could have resulted in the leads breaking off, or in new bends forming in the leads that would create more reflection points, so this was not done. This meant that there would be $10.5 \mathrm{~mm}$ between the end of the insulation on the semi-rigid cable and the laser packaging to create inductance in the input path.

Some steps were taken to reduce the inductance along this uninsulated portion. A layer of single-sided FR4 circuit board was placed between the standard circuit board and the leads to add capacitance (copper side facing away from the leads). This was used to change the permittivity between the lead and the PCB. Figure 4.2 shows the setup for making these connections. After everything was planned out, all that remained was to solder the connections together. A picture of the end result is shown in Figure 4.3.

There are several things to note about the setup. The laser itself is sitting on an aluminum block which serves as a heat sink. The semi-rigid cables are on a copper circuit board elevated to just below the butterfly leads. The left side of the laser has connections useful for monitoring the output power and wavelength. These leads are not used and instead they are insulated from the ground plane. There are two connections for measuring and controlling the temperature; the copper has been cut away from the ground plane to create DC isolation for the negative terminals of these connections. The end of the semi-rigid cable insulation goes right up to the butterfly leads. All of the 
semi-rigid cables are soldered to the ground plane right at the end going to the butterfly leads to reduce parasitic inductance; they are also soldered at the end leading off of the ground plane to create mechanical stability.

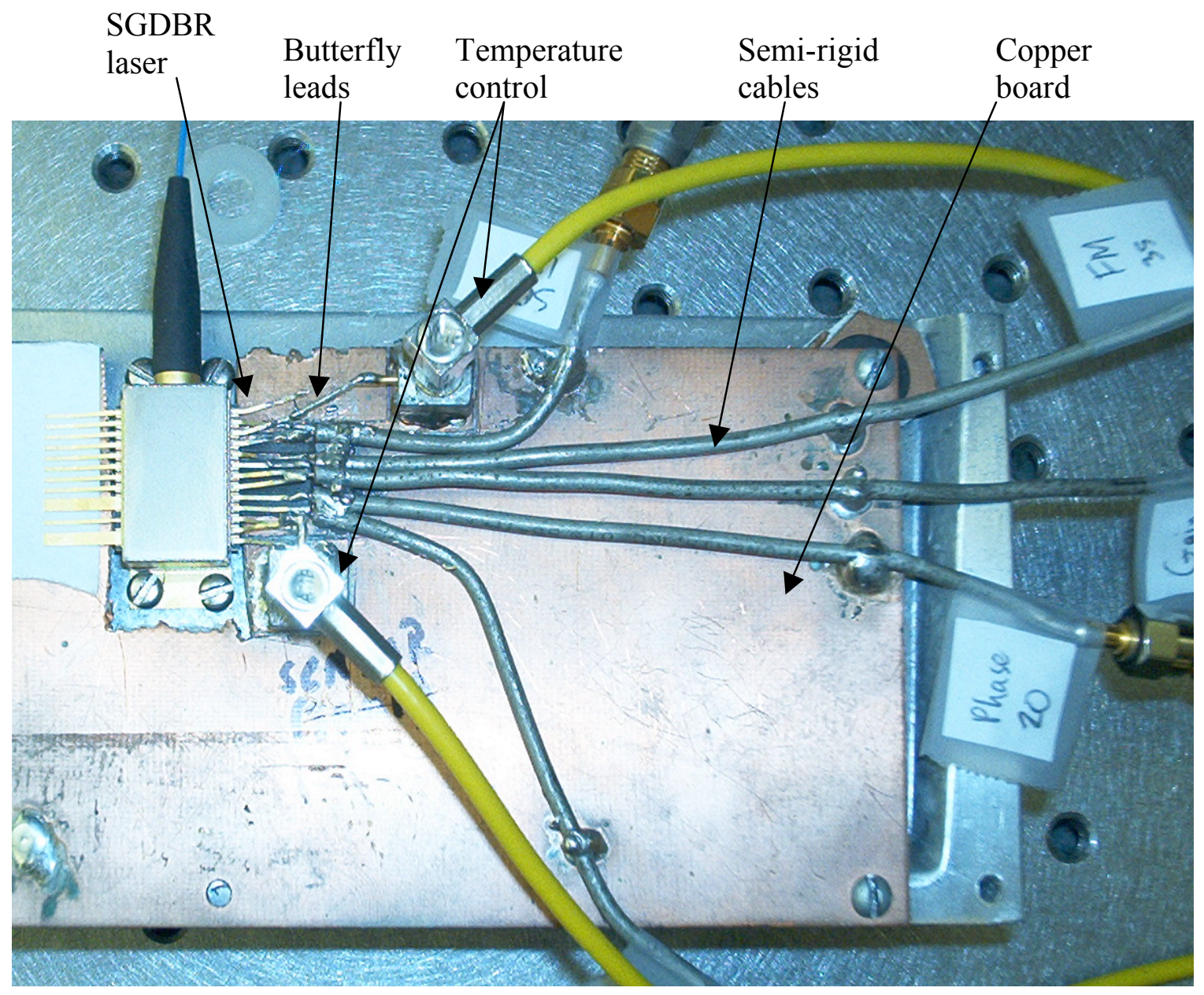

Figure 4.3: Setup for the packaged laser. 


\subsection{Laser DC Characterization}

The first step with this setup was to verify the basic DC functionality of the laser. To do this, the output of the laser was fed into an OSA, and the peak power and wavelength were recorded. The measurement setup is in Figure 4.4.

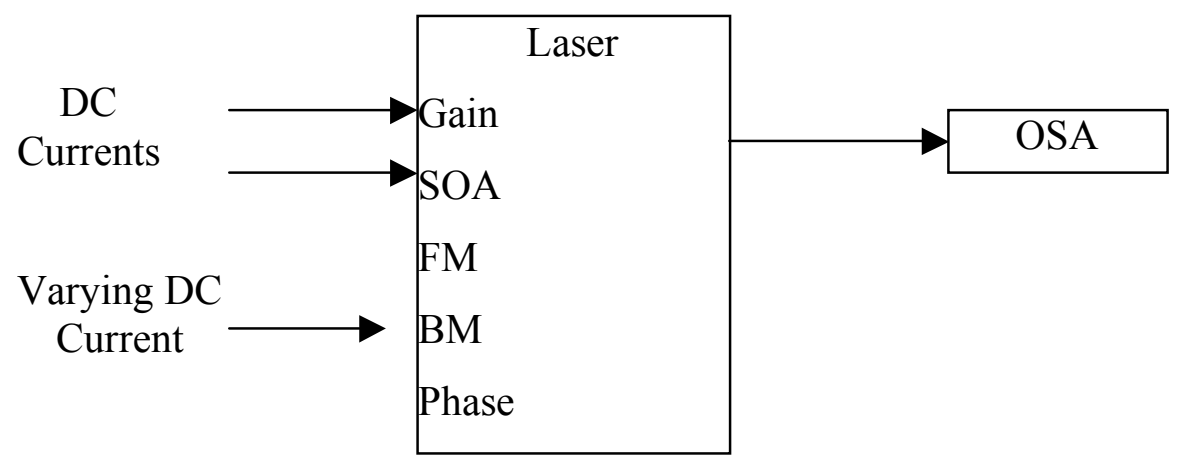

Figure 4.4: Block diagram for DC characterization of the SGDBR laser. A DC current of $100 \mathrm{~mA}$ is sent into the gain segment, $150 \mathrm{~mA}$ is sent into the SOA, and a DC current is sent into one of the FM, BM, or phase sections. The power and wavelength values from the OSA are recorded.

As the current is increased, there is very little wavelength change in the output until there is a mode change in the laser, which causes a jump in wavelength of almost 6 nm, as shown in Figure 4.5. The power also changed very little, especially within each wavelength range, as shown in Figure 4.6. The OSA only has a resolution of $0.1 \mathrm{~dB}$, which causes the change in power to appear more sudden than it actually is. 


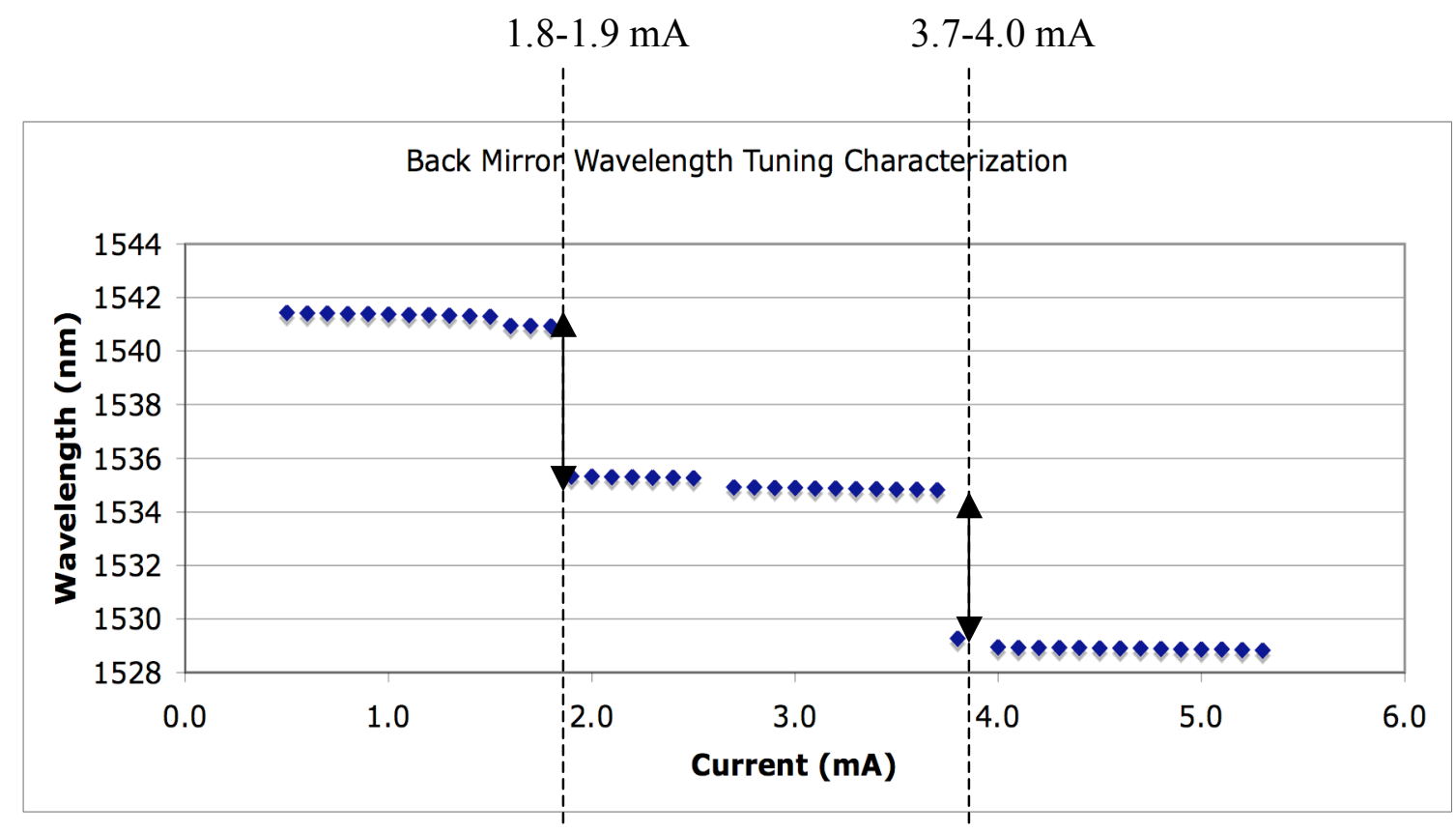

Figure 4.5: Wavelength as a function of back mirror current with $\mathrm{I}_{\text {gain }}=100 \mathrm{~mA}$, $\mathrm{I}_{\mathrm{SOA}}=150 \mathrm{~mA}, \mathrm{I}_{\mathrm{FM}}=\mathrm{I}_{\text {phase }}=0 \mathrm{~mA}$. Vertical lines show where the mode switches occur.

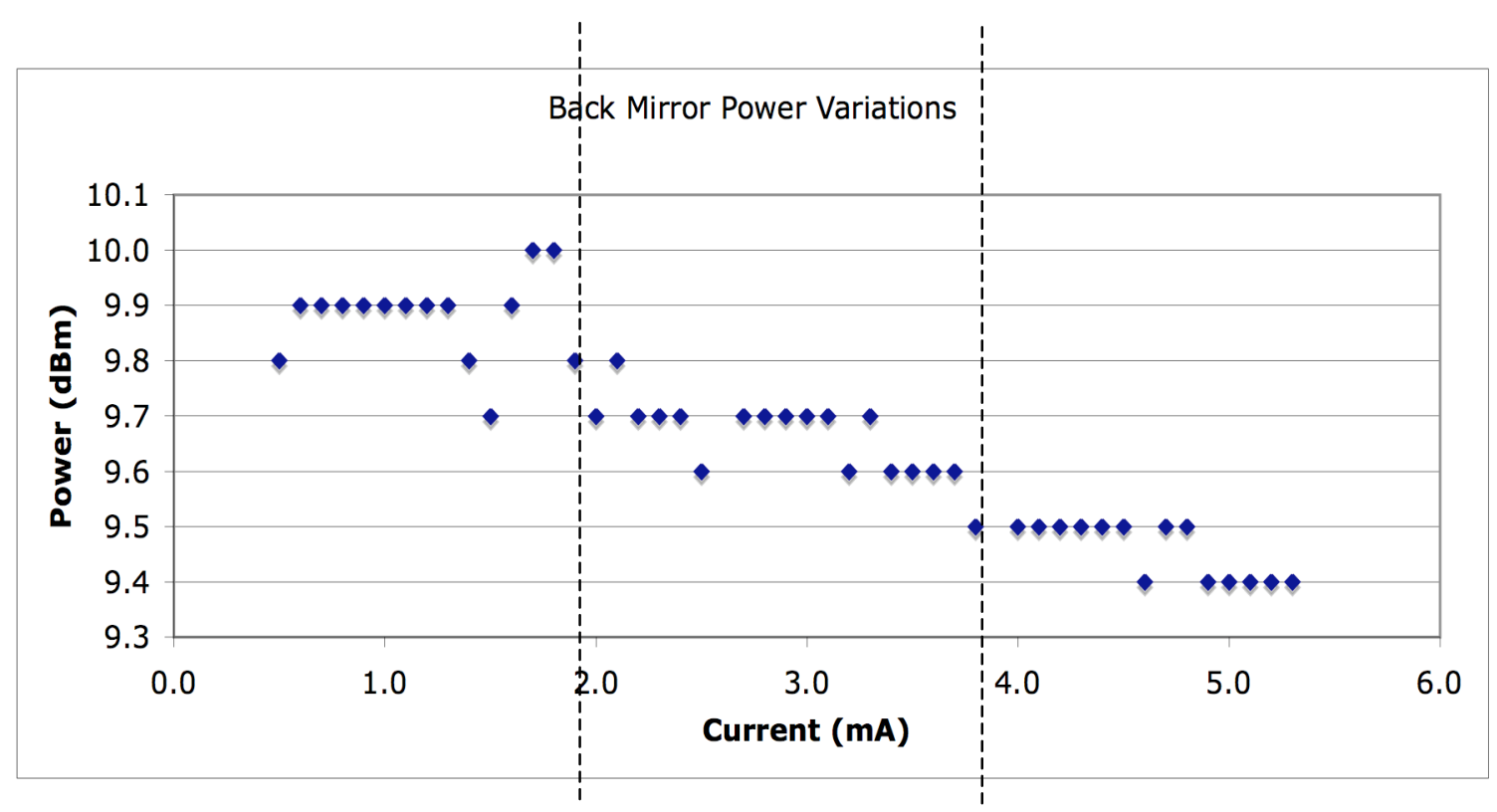

Figure 4.6: Power as a function of back mirror current with $\mathrm{I}_{\text {gain }}=100 \mathrm{~mA}$, $\mathrm{I}_{\mathrm{SOA}}=150 \mathrm{~mA}, \mathrm{I}_{\mathrm{FM}}=\mathrm{I}_{\text {phase }}=0 \mathrm{~mA}$. Vertical lines show where the mode switches occur. 


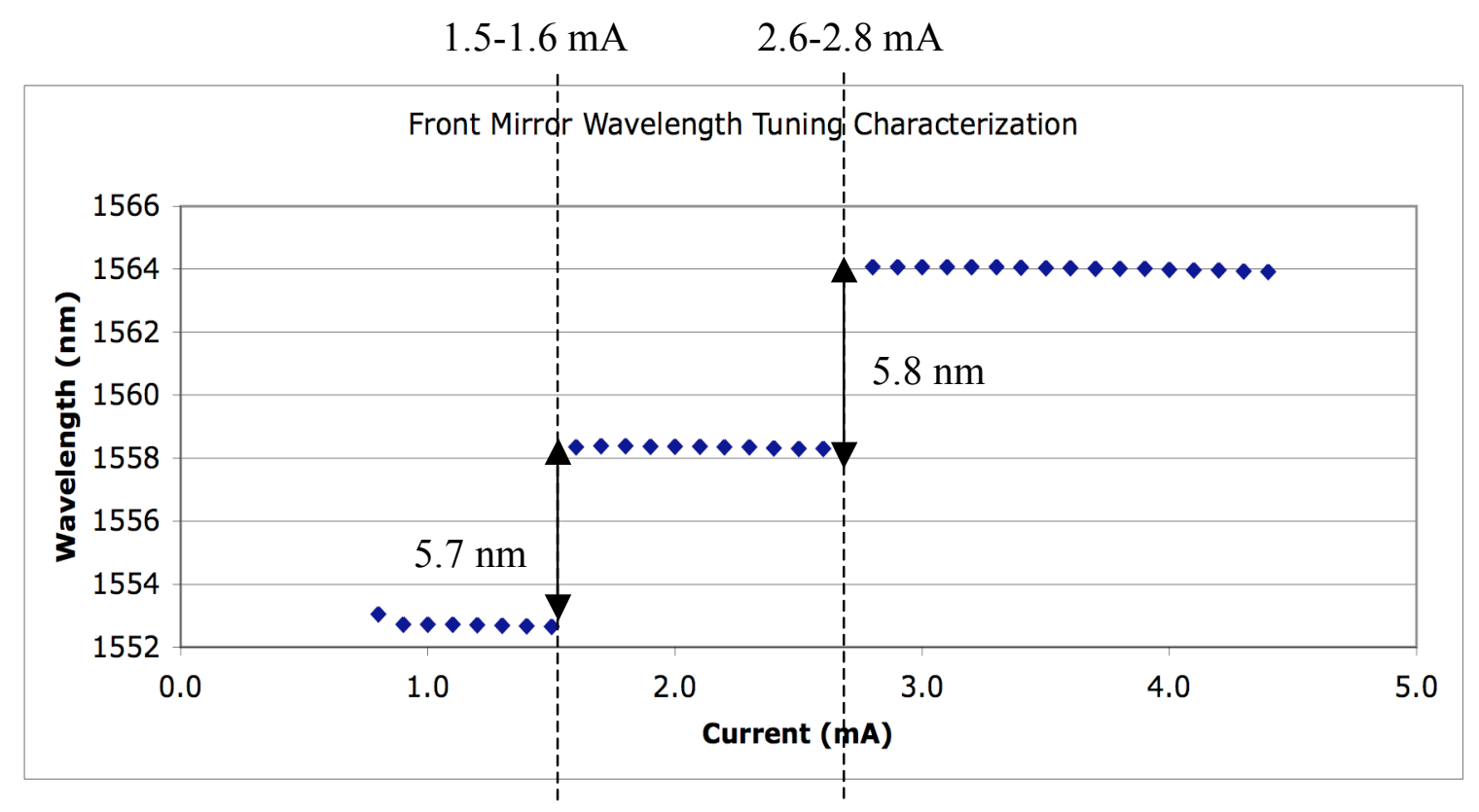

Figure 4.7: Wavelength as a function of front mirror current with $\mathrm{I}_{\text {gain }}=100 \mathrm{~mA}$, $\mathrm{I}_{\mathrm{SOA}}=150 \mathrm{~mA}, \mathrm{I}_{\text {phase }}=\mathrm{I}_{\mathrm{BM}}=0 \mathrm{~mA}$. Vertical lines show where the mode switches occur.

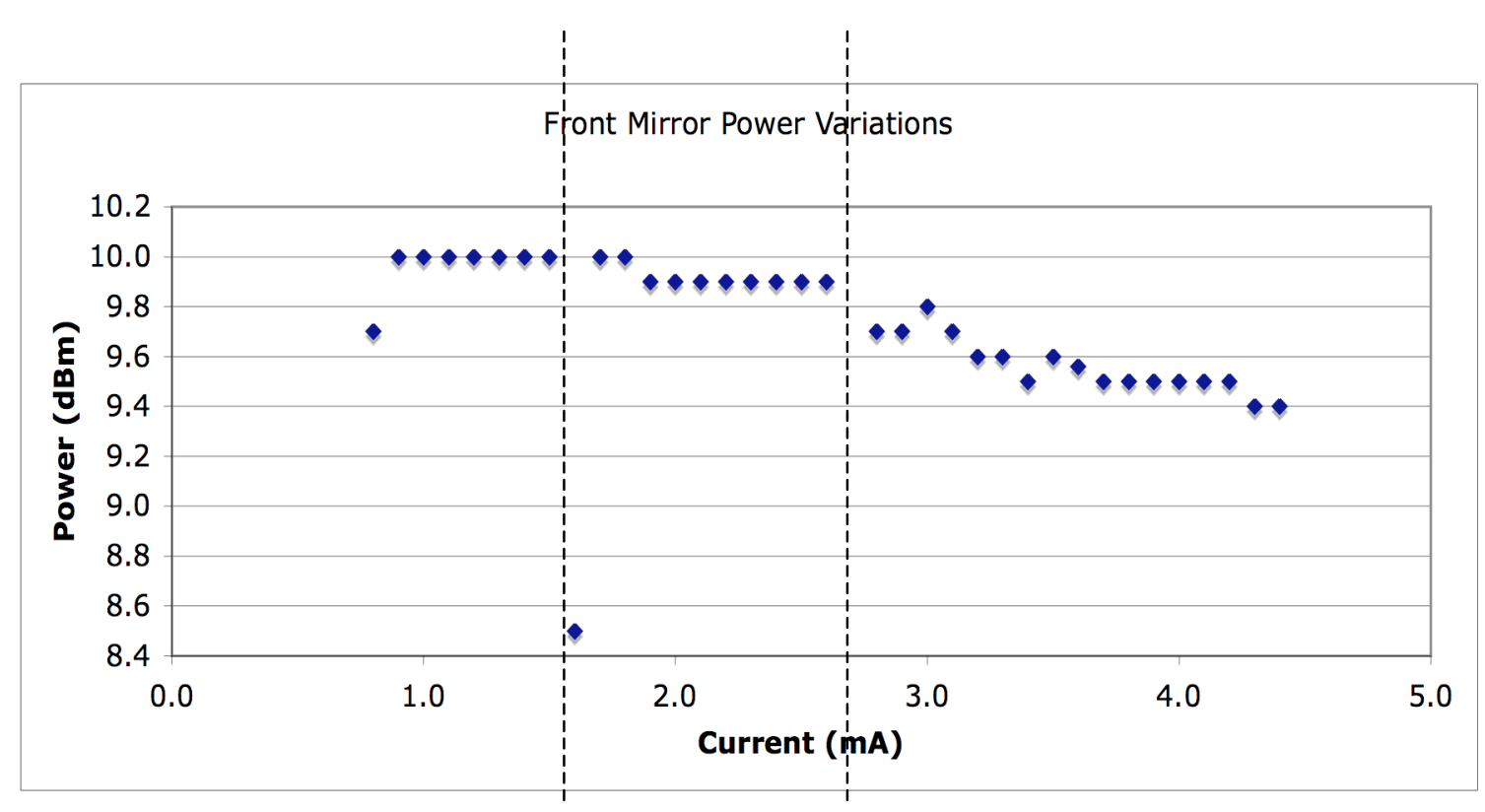

Figure 4.8: Power as a function of front mirror current with $\mathrm{I}_{\text {gain }}=100 \mathrm{~mA}$, $\mathrm{I}_{\mathrm{SOA}}=150 \mathrm{~mA}, \mathrm{I}_{\mathrm{phase}}=\mathrm{I}_{\mathrm{BM}}=0 \mathrm{~mA}$. Vertical lines show where the mode switches occur. 
The front mirror operates like the back mirror, with almost no wavelength change except for the large mode jumps. There was also very little change in output power except when the laser changed modes. This is shown in Figures 4.7 and 4.8.

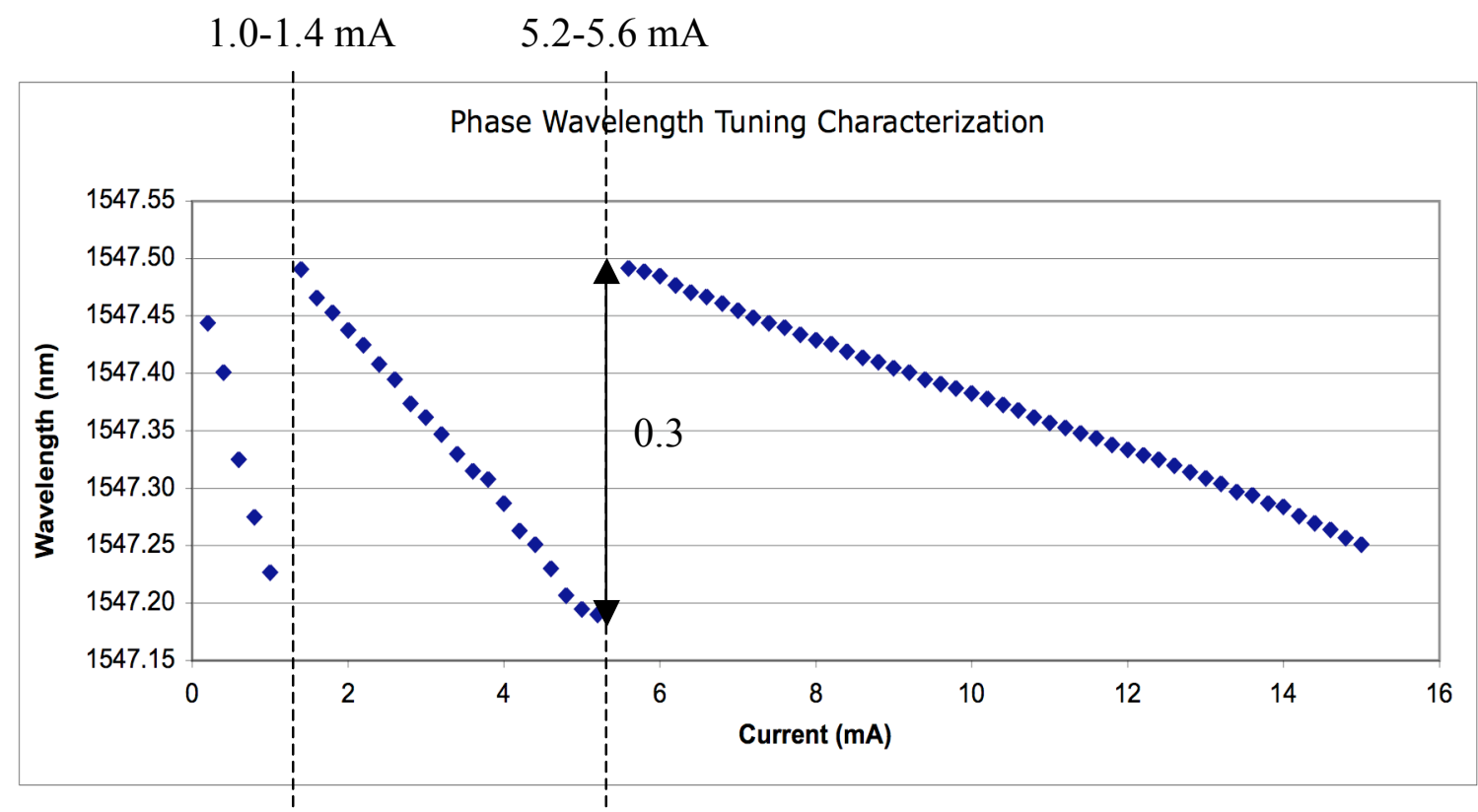

Figure 4.9: Wavelength as a function of phase current with $I_{\text {gain }}=100 \mathrm{~mA}$, $\mathrm{I}_{\mathrm{SOA}}=150 \mathrm{~mA}, \mathrm{I}_{\mathrm{FM}}=\mathrm{I}_{\mathrm{BM}}=0 \mathrm{~mA}$. Vertical lines show where the mode switches occur.

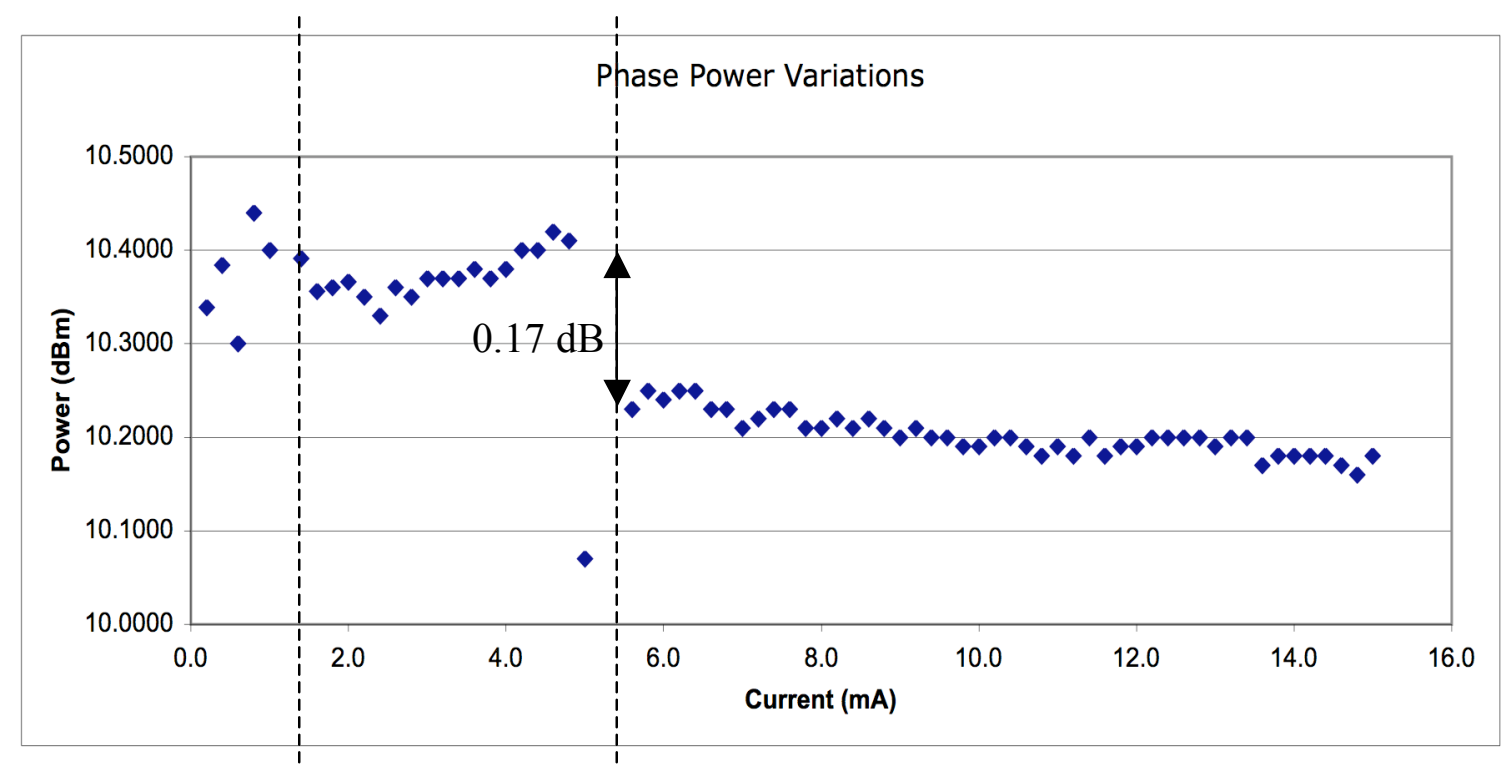

Figure 4.10: Power as a function of phase current with $\mathrm{I}_{\text {gain }}=100 \mathrm{~mA}, \mathrm{I}_{\mathrm{SOA}}=150$ $\mathrm{mA}, \mathrm{I}_{\mathrm{FM}}=\mathrm{I}_{\mathrm{BM}}=0 \mathrm{~mA}$. Vertical lines show where the mode switches occur. 
The laser gradually decreases in wavelength as the phase current is increased, shown in Figure 4.9. At a few points the wavelength jumps back up to its starting value and repeats the cycle. The output power is fairly consistent except for the jump when a new cycle starts, shown in Figure 4.10.

\subsection{Amplitude Modulation Performance}

\subsubsection{Measurement Setup}

An important thing to characterize about this laser is how quickly the inputs can be changed. The technique used for making this measurement was to apply a modulation signal to one of the laser inputs and measure the resulting output amplitude modulation using the ESA. The input modulation frequency is increased; when the output modulation amplitude drops by $3 \mathrm{~dB}$ then the response time of that input can be found.

Figure 4.11 shows the setup for this measurement.

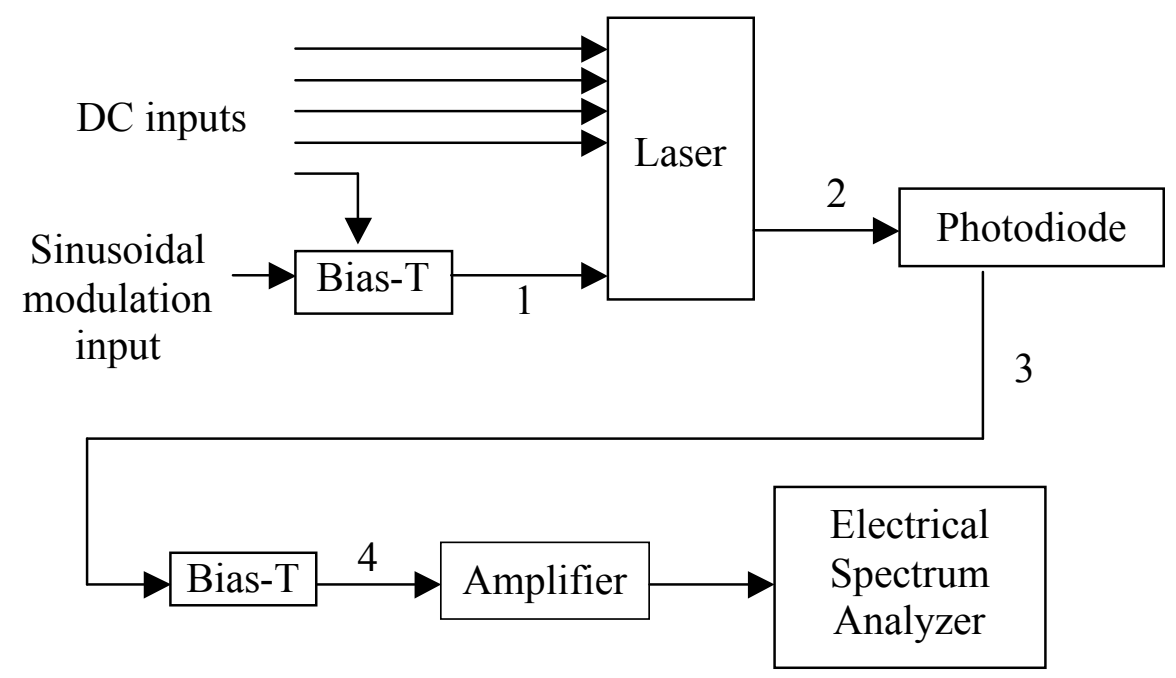

Figure 4.11: Setup for measuring the amplitude modulation response for inputs. Following this diagram is an explanation of the different numbered portions. 
1: This is one of the laser inputs. After the DC and sinusoid portions of the signal are combined through the bias- $\mathrm{T}$, the signal shown in Figure 4.12 is sent into the laser.

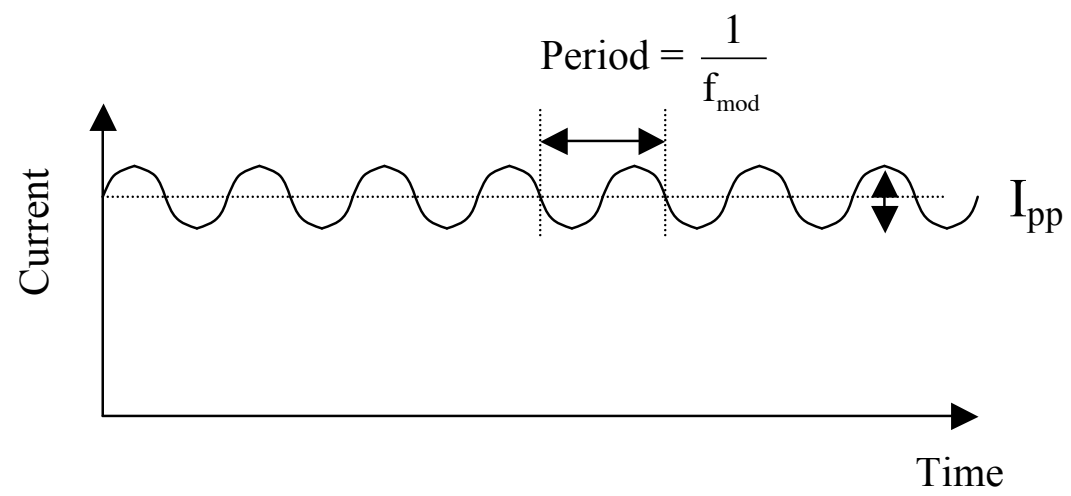

Figure 4.12: Input signal for the section being modulated. Point 1 from Figure 4.11.

2: The laser output will be at the frequency of laser operation, with amplitude modulation occurring at the same frequency as the current modulation input signal. The amplitude of the modulation signal depends on the input being varied. Figure 4.13 shows the electric field of the optical signal.

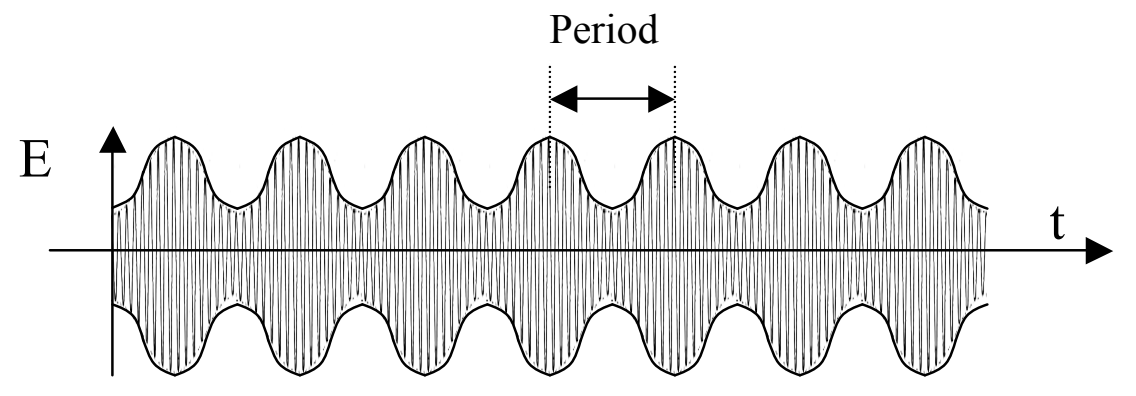

Figure 4.13: Point 2 from Figure 4.11. $194 \mathrm{THz}$ signal from the laser being amplitude modulated at a frequency less than $1 \mathrm{GHz}$ 
3: The photodiode converts the optical signal to a current, with a conversion factor of $0.687 \mathrm{~A} / \mathrm{W}$. The photodiode had a $-5 \mathrm{~V}$ bias applied to it. The photodiode is too slow to respond to the $194 \mathrm{THz}$ signal coming from the laser, so it acts as an envelope detector. Figure 4.14 shows the output from the photodiode.

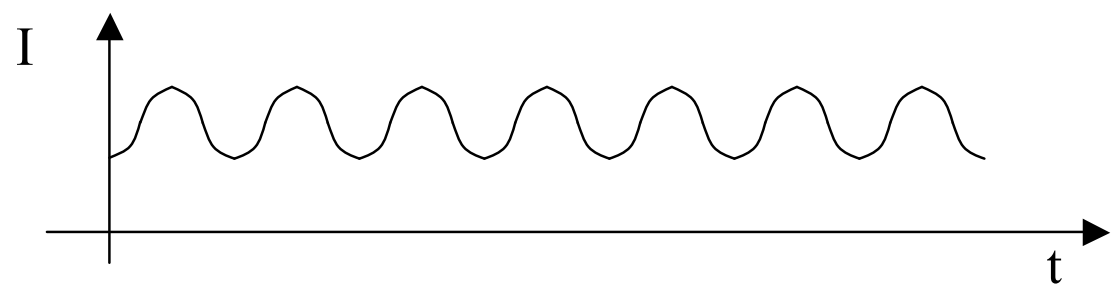

Figure 4.14: Point 3 from Figure 4.11. Output from the photodiode.

4: The bias-T is used to eliminate the DC portion of the signal and leave the modulation, allowing the photodetector to have a reverse-bias voltage without carrying through to the amplifier and ESA. The result is shown in Figure 4.15. This signal is sent into the amplifier (characterized as shown in Figure 4.16) and then to the ESA.

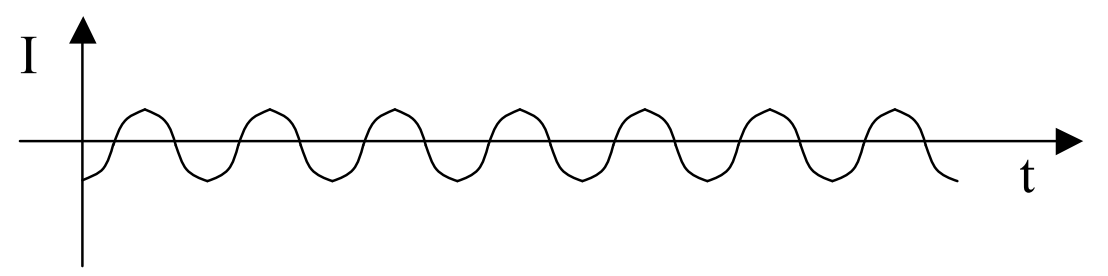

Figure 4.15: Point 4 from Figure 4.11. Photodiode output, after being sent through a bias-T. 


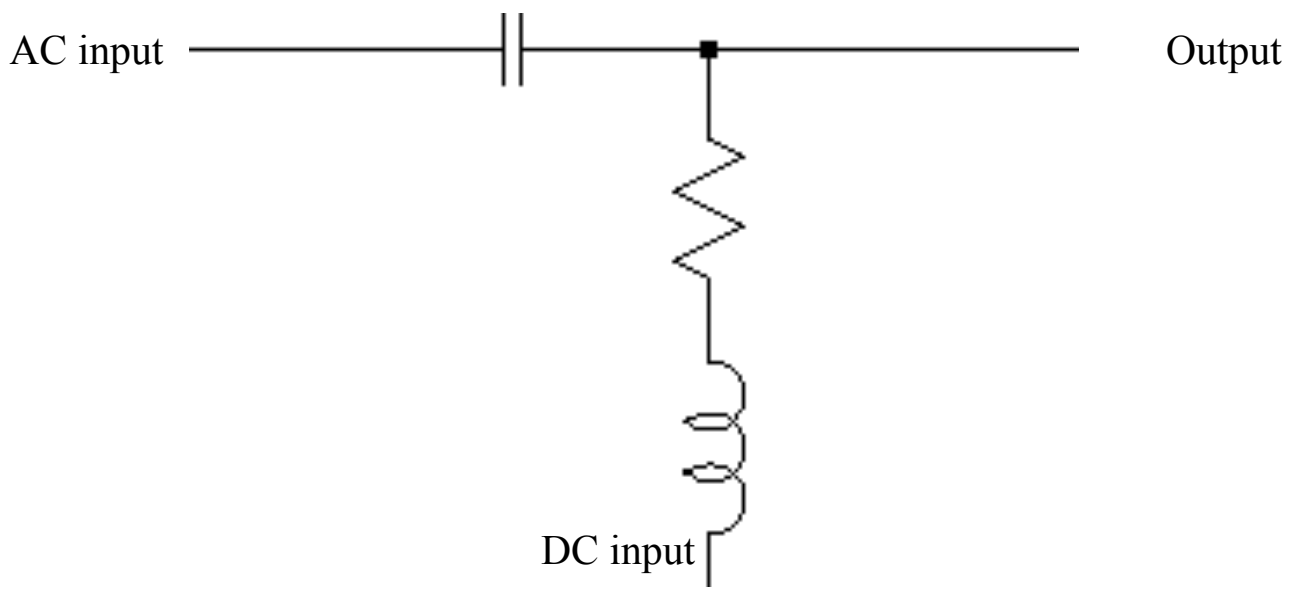

Figure 4.16: Bias-T used in the setup shown in Figure 4.11.

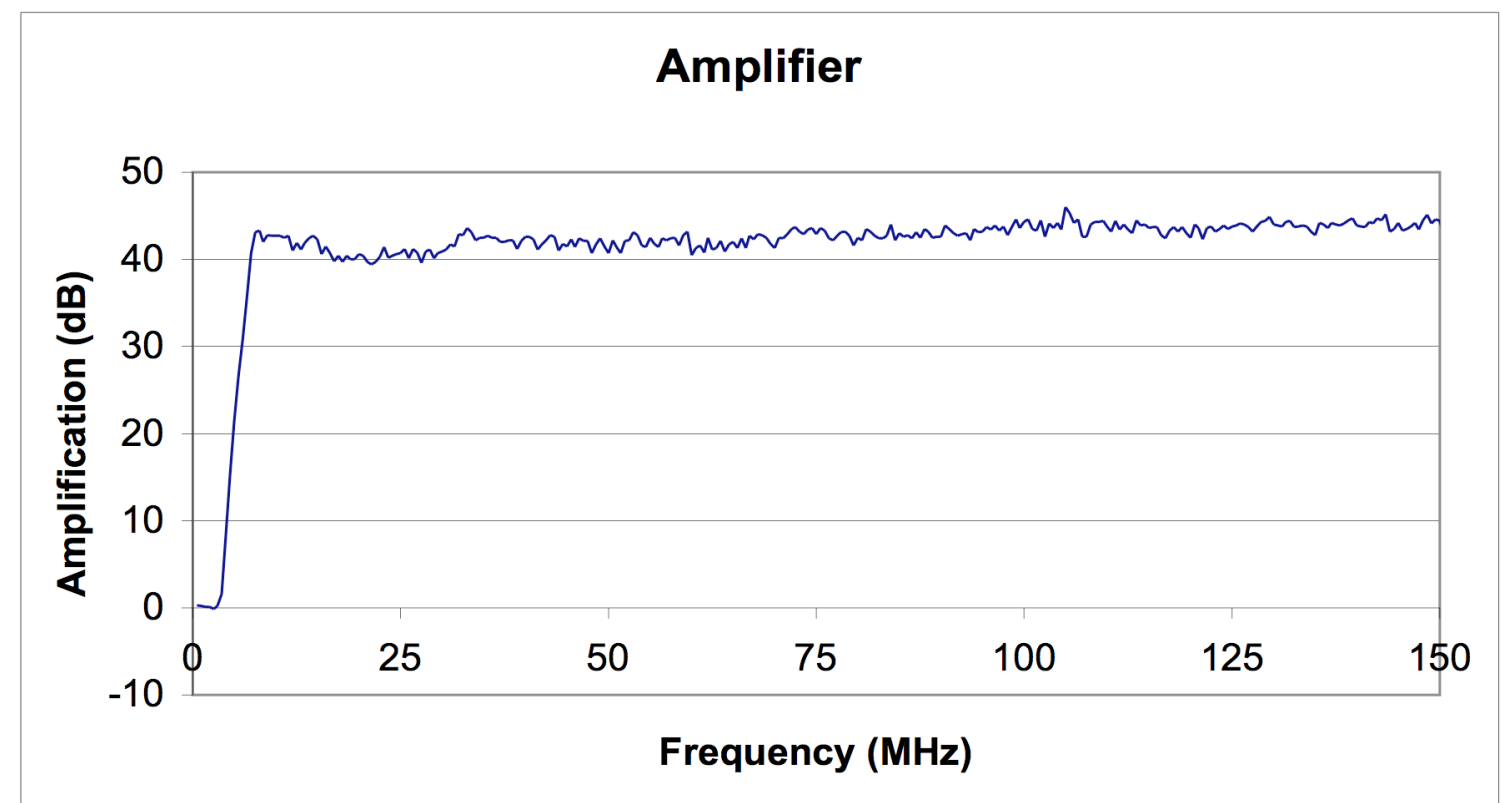

Figure 4.17: Characterization of the amplifier used for the setup shown in Figure 4.11 .

\subsection{Frequency Modulation Performance}

\subsubsection{Viewing the Frequency Modulation Response on the OSA}

To view the effects of frequency on phase section modulation, the phase section was modulated with a square wave and the OSA display was viewed. The square wave 
was sent into the phase section to quickly change between two wavelengths. Figure 4.18 shows the setup, and Figures 4.19 and 4.20 show the results.

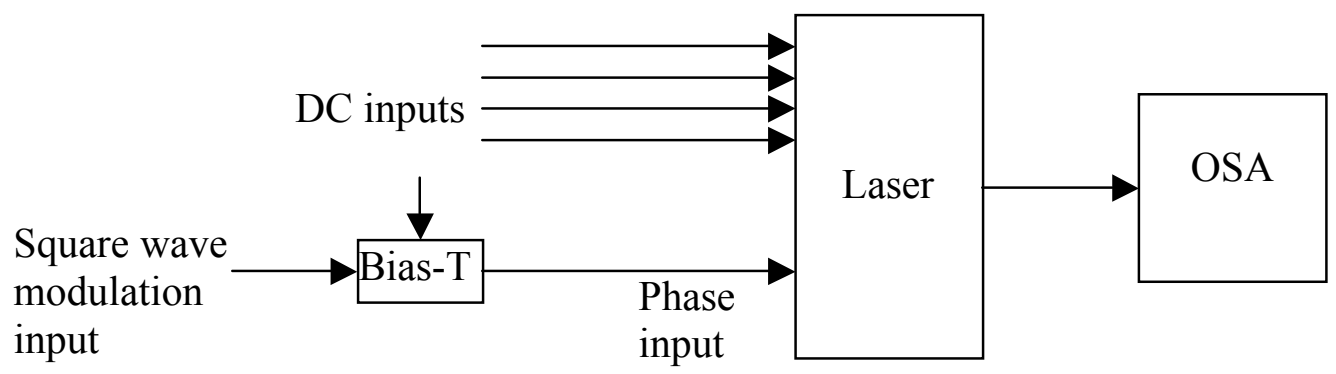

Figure 4.18: Observing the effects of frequency modulation. $\mathrm{I}_{\mathrm{FM}}=20.0 \mathrm{~mA}$, $\mathrm{I}_{\mathrm{BM}}=30.0 \mathrm{~mA}, \mathrm{I}_{\text {Gain }}=100 \mathrm{~mA}, \mathrm{I}_{\mathrm{SOA}}=150 \mathrm{~mA}$, I $\mathrm{I}_{\text {Phase }}=5.5 \mathrm{~mA} \mathrm{DC}, 2 \mathrm{~V}$ square wave sent through a $30 \mathrm{~dB}$ attenuator, combined with a bias-T

By increasing the modulation frequency, the wavelength separation between the peaks decreases, an indication that the current is switching back and forth more quickly than the phase section can respond. As the frequency is increased further, there should be an even smaller change in the wavelength shift.

These results show the effect of frequency modulation, but the results are not good for accurately quantifying how quickly the laser can be modulated. The 3-dB corner frequency appears to be somewhere around 50-70 MHz. The next step is to quantify when the wavelength is unable to shift fast enough so that the response time for the phase, front mirror, and back mirror inputs can be determined. 


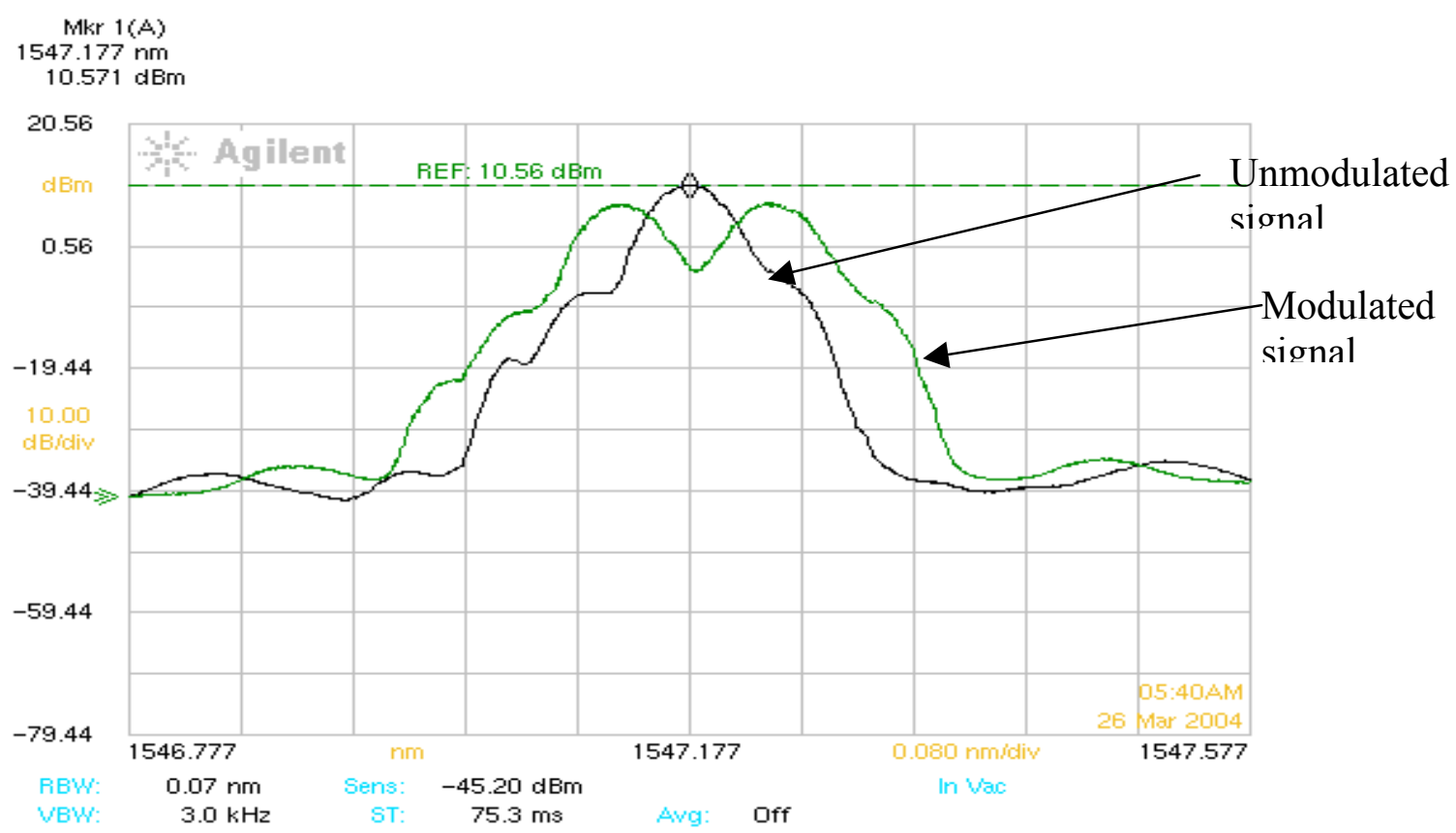

Figure 4.19: $143 \mathrm{KHz}$ modulated phase input causes the laser to switch between two wavelengths. The unmodulated signal is shown for comparison. The OSA is slow enough ( $13.3 \mathrm{~Hz}$ repetition rate) that the modulated signal is averaged and shows both wavelengths. The plot is a comparison of power versus wavelength.

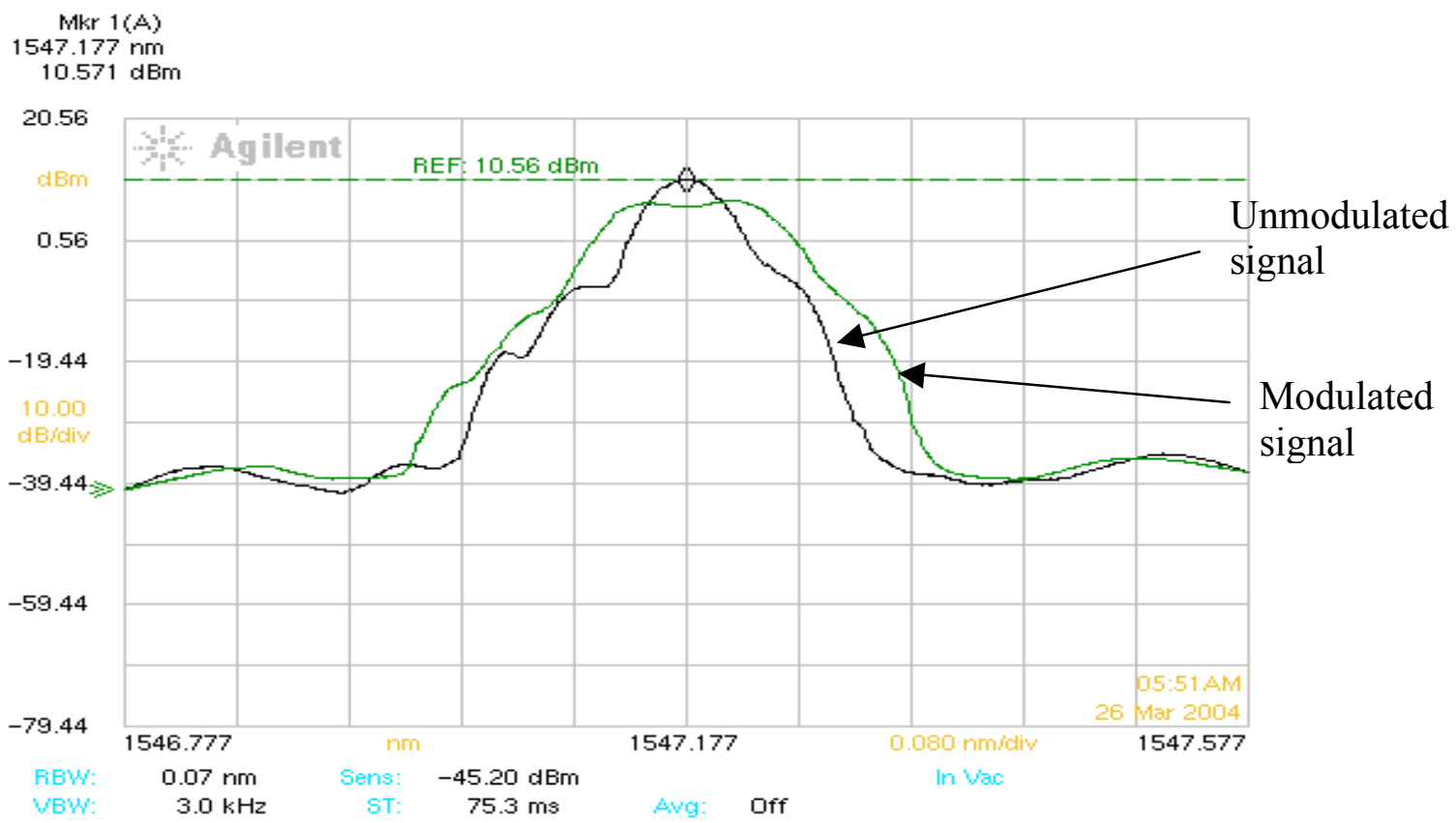

Figure 4.20: $50 \mathrm{MHz}$ modulated phase input causes the laser to switch between two wavelengths. The peaks have moved closer together compared to their position in Figure 4.19. 


\subsubsection{Measurement Setup}

To determine exactly how the modulation frequency affects the change in wavelength, a narrow wavelength-dependent filter in the form of a Mach-Zehnder interferometer was used. With this approach, a modulation signal is sent into a laser segment, which causes the wavelength of the laser output to change. The changing wavelength results in a change in power as the laser output passes through the filter, converting the frequency modulation into amplitude modulation. This is illustrated in Figure 4.23.

By changing the wavelength of the laser, the power that comes out from the filter changes. This signal is then sent through a photodiode, an amplifier, and into the ESA, using the same process as amplitude modulation testing. The measurement is read off the ESA in the same way as it was for amplitude modulation measurements, reading the power for the modulation frequency. A block diagram of the setup is shown in Figure

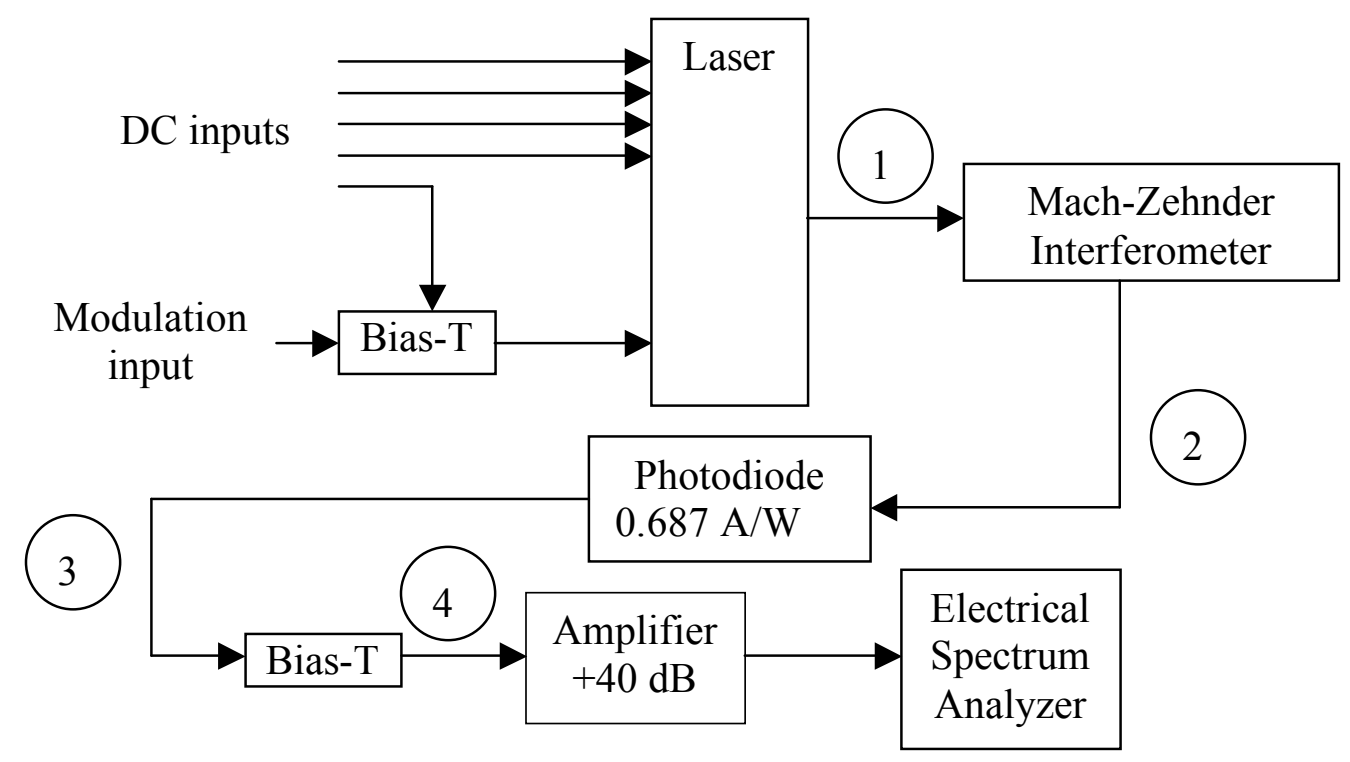

Figure 4.21: Setup for frequency modulation measurements. 
1: This is the modulated output from the laser. It has the same form as the laser output from amplitude modulation measurements as shown in Figure 4.15 (p. 54).

2: The filter converts frequency modulation into amplitude modulation. A change in wavelength results in a change in the power transmitted. For example, a signal at $1547.219 \mathrm{~nm}$ will have $24 \%$ of its power transmitted, while a signal at $1547.298 \mathrm{~nm}$ will have $12 \%$ of its power transmitted through the filter. This step changes wavelength modulation into amplitude modulation which can be measured more easily.

3: The photodiode converts the signal from an optical signal to an electrical signal.

4: The bias-T is used to center the signal around $\mathrm{DC}$, so that only the modulation signal reaches the ESA.

\subsubsection{Filter (Mach-Zehnder Interferometer)}

The filter is the key to the frequency modulation measurements. The filter is created using a Mach-Zehnder interferometer, shown in Figure 4.22. The interferometer is a passive device that splits the light into two paths and then recombines it. For this interferometer, the time difference between the two paths is approximately $12 \mathrm{um}$. This causes the two signals to interfere constructively or destructively when they recombine, depending on their phase difference. The phase difference between the two paths varies when the laser wavelength is changed. 


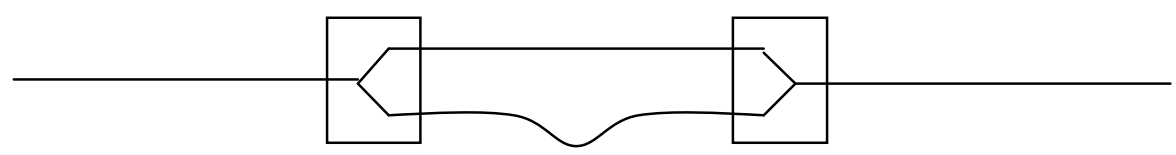

Figure 4.22: Mach-Zehnder interferometer. Light enters through one fiber, goes through a splitter into two separate fibers of slightly different lengths, then gets recombined into a single fiber. The filter has a free spectral range of $50 \mathrm{GHz}$.

By changing the current input to one of the wavelength-controlling sections, the laser wavelength changes, which causes a different level of interference from the interferometer. This causes a different amount of power to come out from the interferometer, effectively translating wavelength modulation into amplitude modulation.

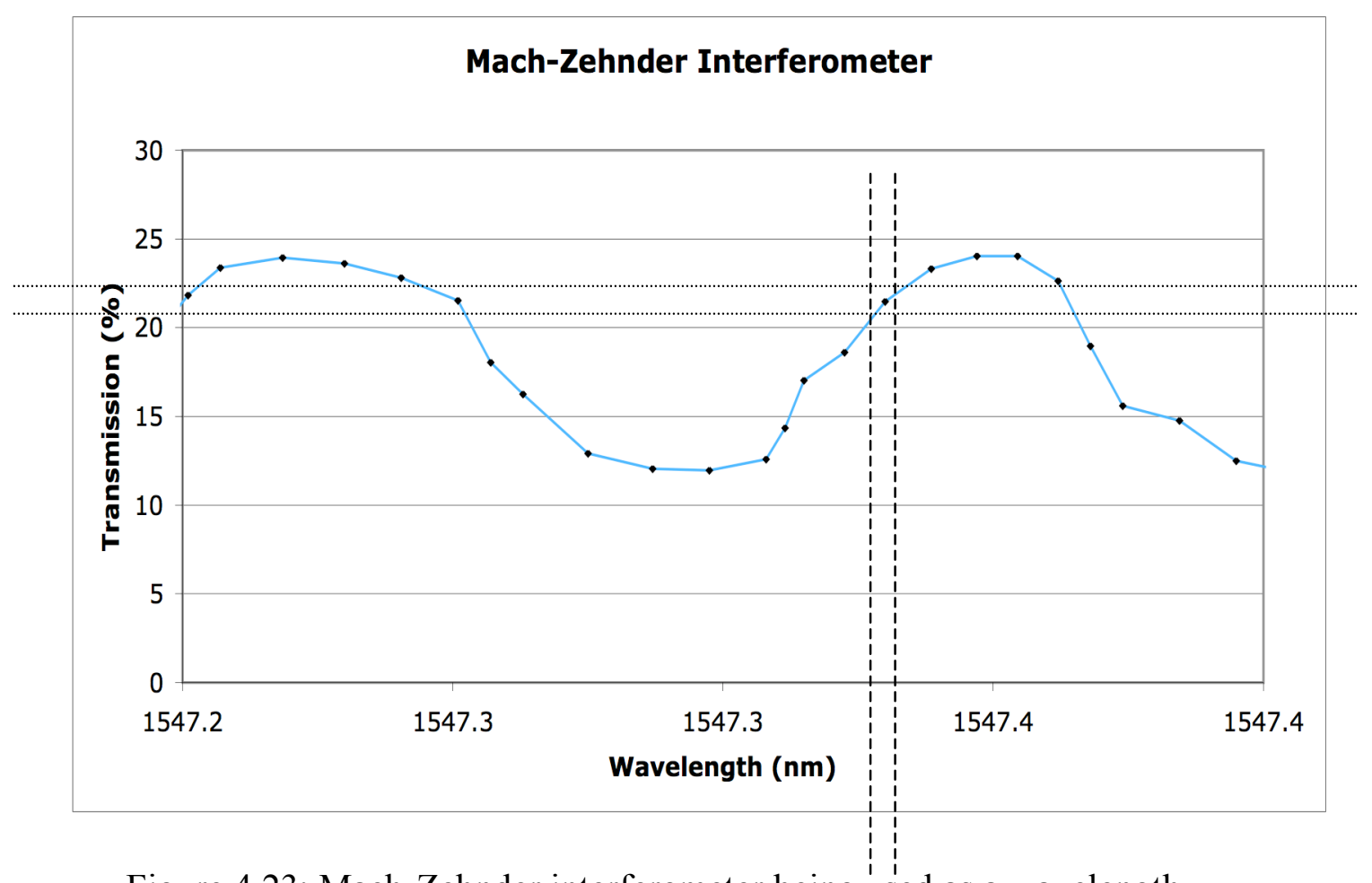

Figure 4.23: Mach-Zehnder interferometer being used as a wavelength discriminator. As the wavelength changes, the power exiting from the interferometer changes, translating frequency modulation into amplitude modulation. The dashed lines in the figure show the actual wavelength change that occurs when the phase section is modulated and sent through the interferometer. 
Figure 4.23 shows the optical transmission through the interferometer at different wavelengths around the bias point used for measurements of the phase section. With a 50 $\mu \mathrm{A}$ peak input current swing, the power allowed through the filter changes from $20.76 \%$ to $21.94 \%$, which is a change of $1.18 \%$ of the total laser output power. This means that the change in wavelength of the laser will cause a change in the power output, converting wavelength modulation of the laser to amplitude modulation. This might seem like a small change in power, but the output from the laser changes by about $0.03 \%$ when it does not pass through the interferometer.

\subsubsection{Choosing a Bias Point}

To use the Mach-Zehnder interferometer for frequency modulation measurements, a bias point had to be picked for the measurements. The setup for doing this is mostly the same as shown in Figure 4.20. However, instead of changing the modulation frequency of the input, the DC current is changed and the modulation frequency is kept at a constant $10 \mathrm{MHz}$. The goal is to find a DC bias point that has a high output power while avoiding mode switches. These bias points will indicate setups where the slope of the filter (from Figure 4.23) is relatively large in magnitude. At these bias points a specific change in wavelength will result in a larger change in output power than the same change in wavelength at other bias points. 


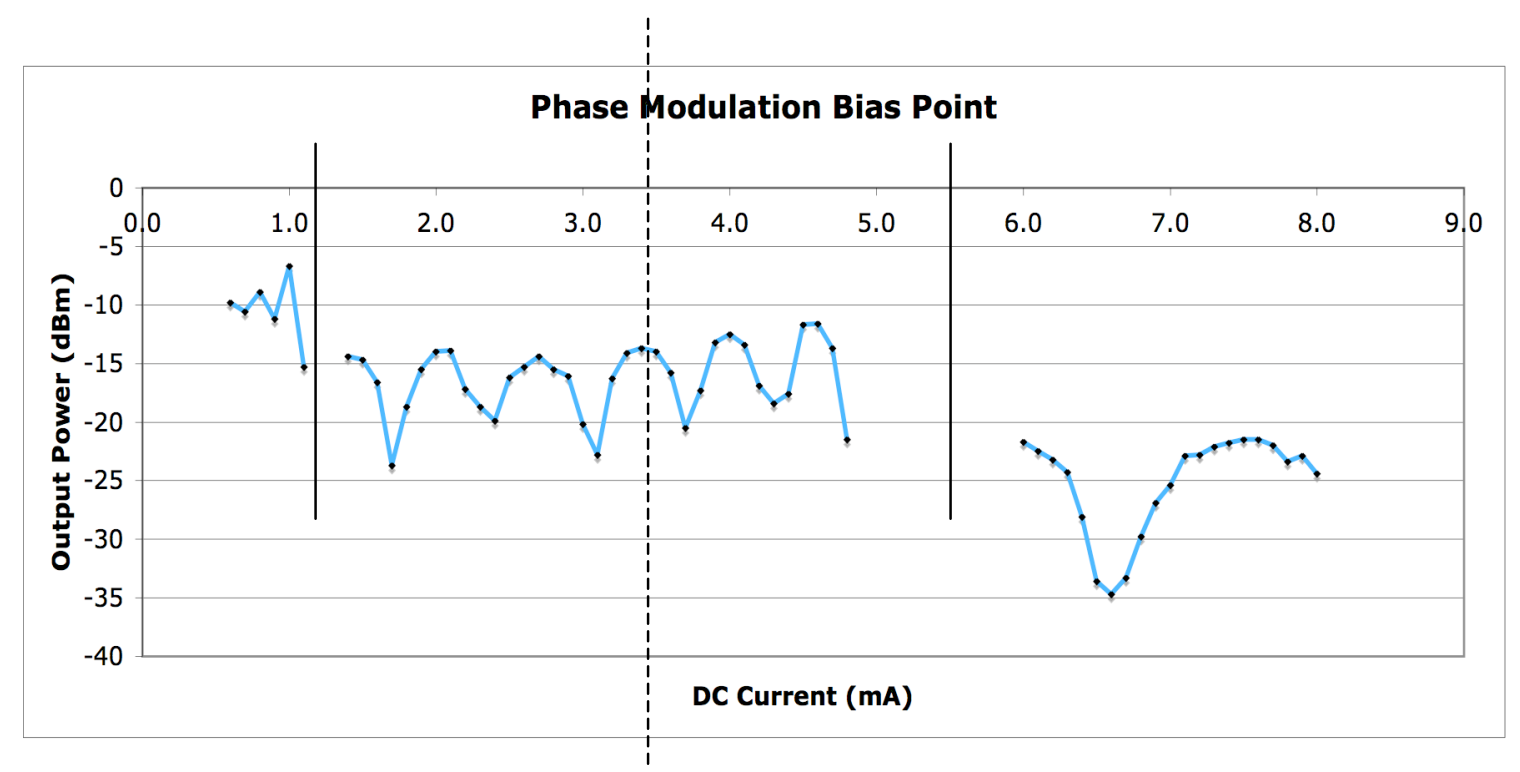

Figure 4.24: Picking a DC bias point for the phase section. Using $3.4 \mathrm{~mA}$ (shown by the dashed line) lines up with a peak and avoids mode changes (solid lines). $\mathrm{I}_{\mathrm{SOA}}=150 \mathrm{~mA} ; \mathrm{I}_{\text {gain }}=100 \mathrm{~mA} ; \mathrm{I}_{\mathrm{FM}}=\mathrm{I}_{\mathrm{BM}}=0 \mathrm{~mA} ; \mathrm{I}_{\text {phase }}(\mathrm{AC})=-70$ dBm, $10 \mathrm{MHz}$.

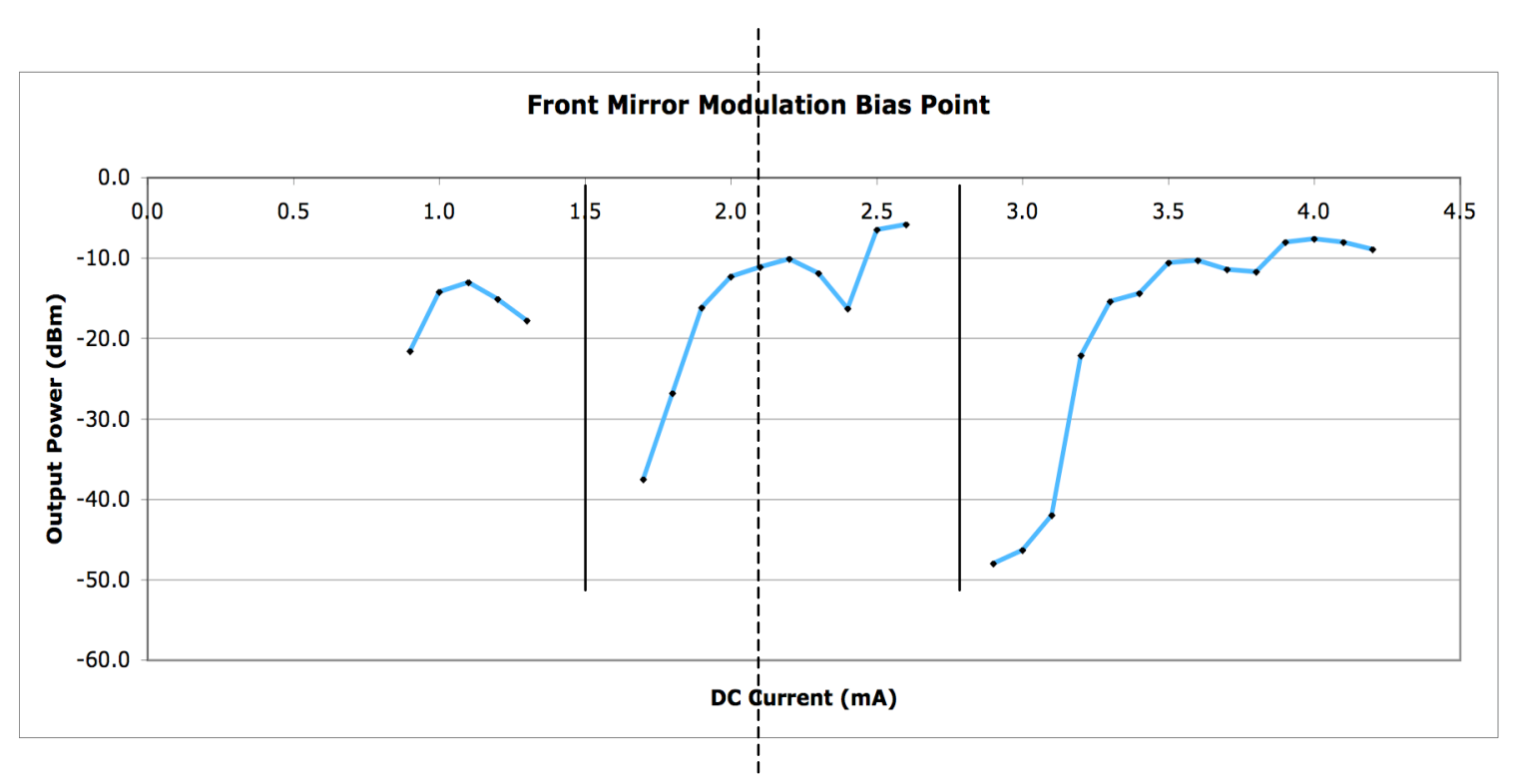

Figure 4.25: Picking a DC bias point for the front mirror section. Using $2.1 \mathrm{~mA}$ (shown by the dashed line) lines up with a peak and avoids mode changes (solid lines). $\mathrm{I}_{\mathrm{SOA}}=150 \mathrm{~mA} ; \mathrm{I}_{\text {gain }}=100 \mathrm{~mA} ; \mathrm{I}_{\text {phase }}=\mathrm{I}_{\mathrm{BM}}=0 \mathrm{~mA} ; \mathrm{I}_{\mathrm{FM}}$ $(\mathrm{AC})=-70 \mathrm{dBm}, 10 \mathrm{MHz}$. 


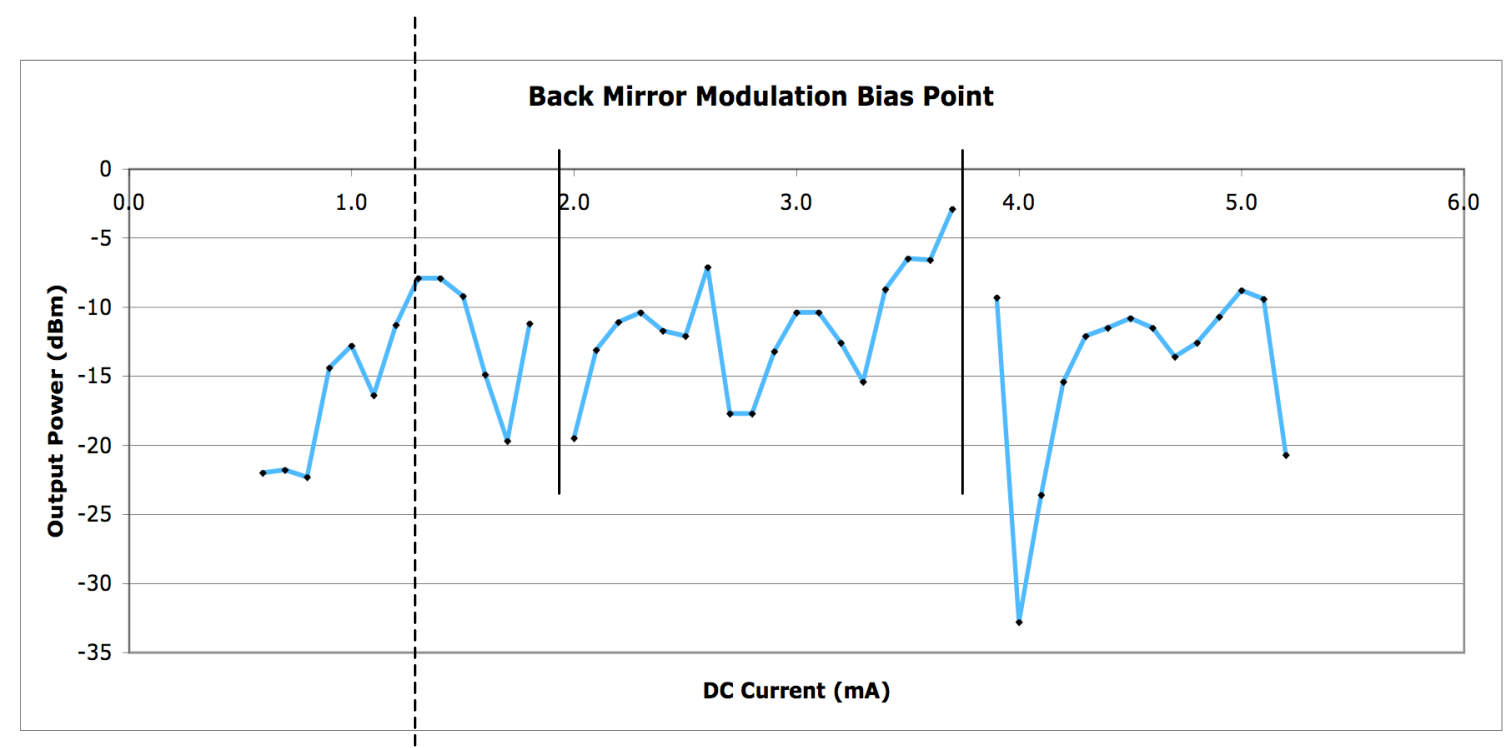

Figure 4.26: Picking a DC bias point for the back mirror section. Using $3.4 \mathrm{~mA}$ (shown by the dashed line) lines up with a peak and avoids mode changes (solid lines). $\mathrm{I}_{\mathrm{SOA}}=150 \mathrm{~mA} ; \mathrm{I}_{\text {gain }}=100 \mathrm{~mA} ; \mathrm{I}_{\mathrm{FM}}=\mathrm{I}_{\text {phase }}=0 \mathrm{~mA} ; \mathrm{I}_{\mathrm{BM}}$ $(\mathrm{AC})=-70 \mathrm{dBm}, 10 \mathrm{MHz}$.

Using the setup in Figure 4.21 (p. 58), data was collected, and peaks were found as indicated by the vertical lines in Figures 4.24, 4.25, and 4.26, and shown in Table 4.1. These were used as the bias points for the amplitude modulation and frequency modulation measurements, and also later on for the linewidth measurements.

\begin{tabular}{|l|r|}
\hline \multicolumn{1}{|c|}{ Input } & Bias Current (mA) \\
\hline Phase & 3.4 \\
\hline Front Mirror & 2.1 \\
\hline Back Mirror & 1.3 \\
\hline
\end{tabular}

Table 4.1: DC bias currents used for wavelength-controlling segments for amplitude modulation, frequency modulation, and linewidth measurements. 


\subsection{Modulation Measurement Results}

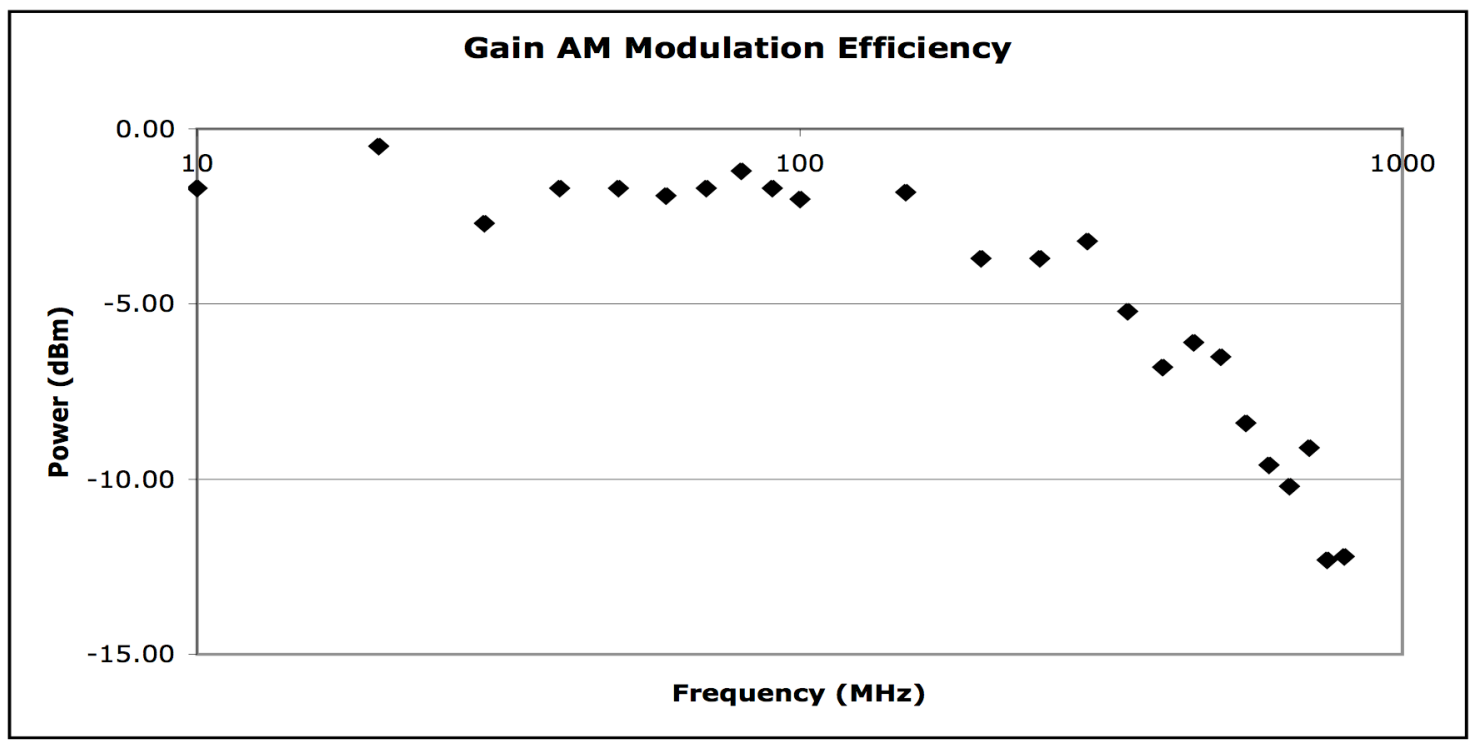

Figure 4.27: Amplitude modulation of Gain section has a $3 \mathrm{~dB}$ frequency of 200 MHz. $\mathrm{I}_{\mathrm{BM}}=23.6 \mathrm{~mA}, \mathrm{I}_{\mathrm{FM}}=41.2 \mathrm{~mA}, \mathrm{I}_{\text {phase }}=6.0 \mathrm{~mA}, \mathrm{I}_{\mathrm{SOA}}=150 \mathrm{~mA}$, $I_{\text {gain }}(\mathrm{DC})=100 \mathrm{~mA}, \mathrm{I}_{\text {gain }}(\mathrm{AC})=-30 \mathrm{dBm}$ with frequencies plotted in the figure above.

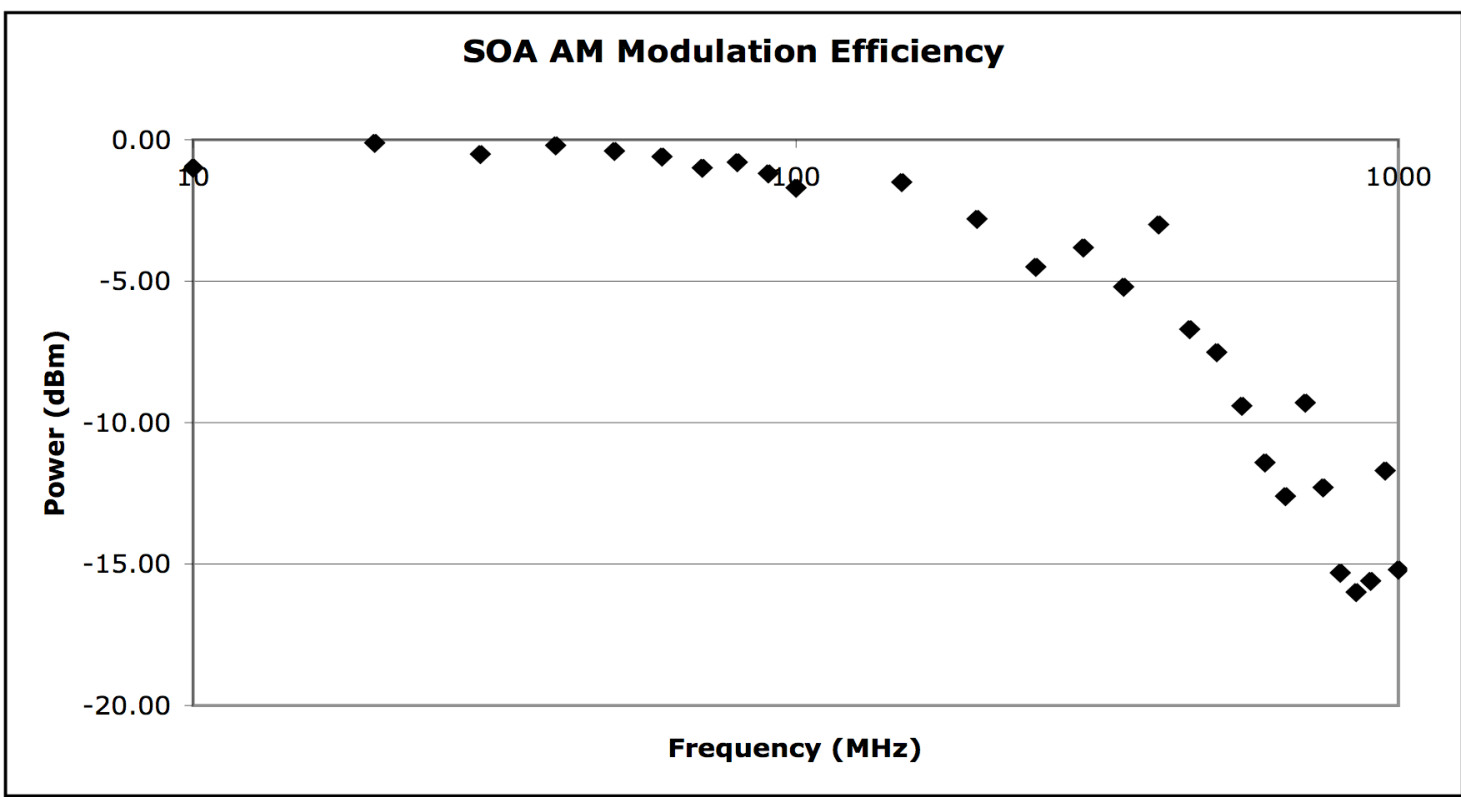

Figure 4.28: Amplitude modulation of SOA section has a $3 \mathrm{~dB}$ frequency of 200 MHz. $\mathrm{I}_{\mathrm{BM}}=23.6 \mathrm{~mA}, \mathrm{I}_{\mathrm{FM}}=41.2 \mathrm{~mA}, \mathrm{I}_{\text {phase }}=6.0 \mathrm{~mA}, \mathrm{I}_{\text {gain }}=100 \mathrm{~mA}$, $\mathrm{I}_{\mathrm{SOA}}(\mathrm{DC})=150 \mathrm{~mA}, \mathrm{I}_{\mathrm{SOA}}(\mathrm{AC})=-30 \mathrm{dBm}$ with frequencies plotted in the figure above. 


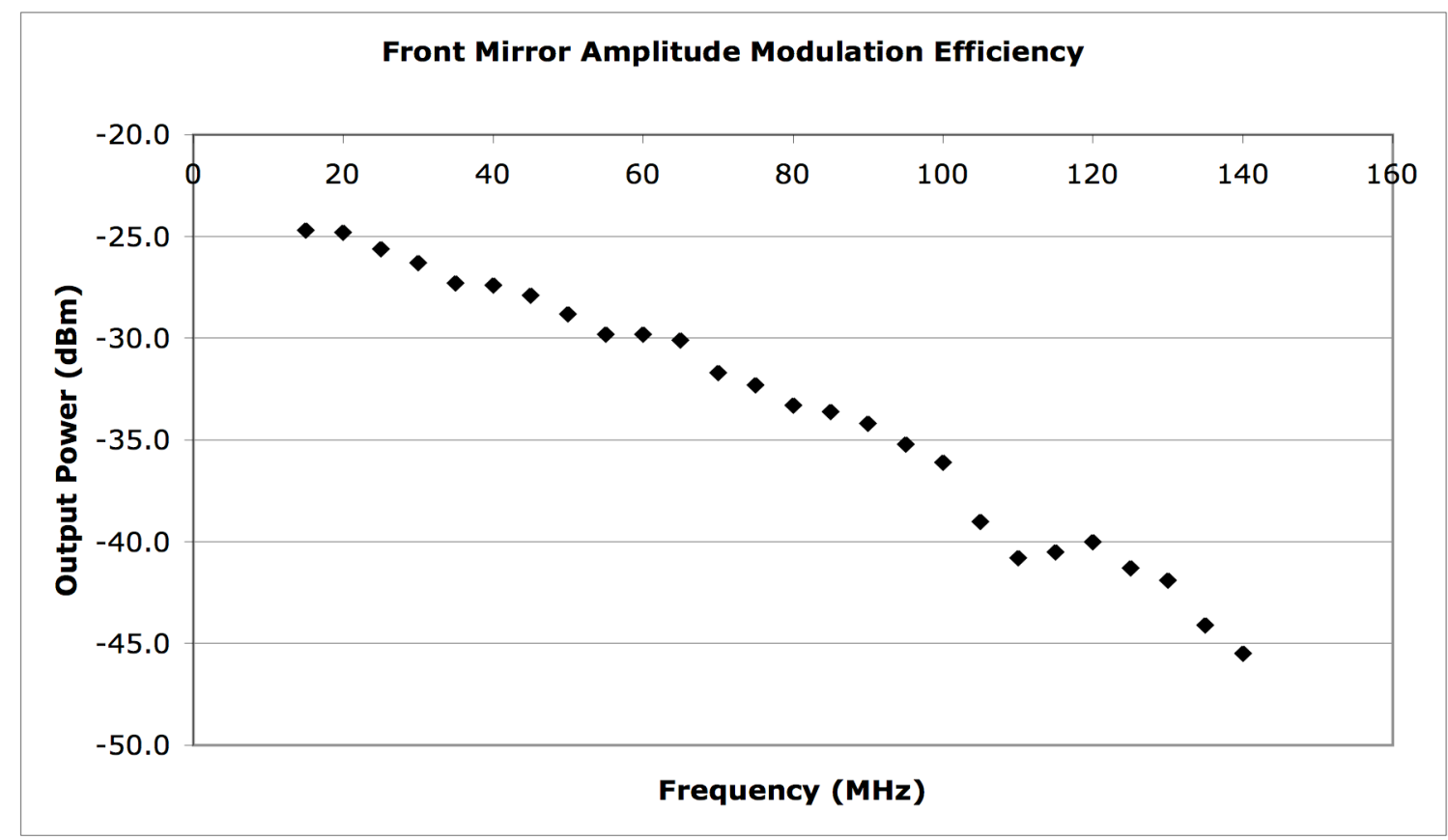

Figure 4.29: Amplitude modulation of the front mirror has a $3 \mathrm{~dB}$ drop at 45

MHz. $\mathrm{I}_{\mathrm{SOA}}=150 \mathrm{~mA} ; \mathrm{I}_{\text {gain }}=100 \mathrm{~mA} ; \mathrm{I}_{\text {phase }}=\mathrm{I}_{\mathrm{BM}}=0 \mathrm{~mA} ; \mathrm{I}_{\mathrm{FM}}(\mathrm{DC})$

$=2.1 \mathrm{~mA} ; \mathrm{I}_{\mathrm{FM}}(\mathrm{AC})=-70 \mathrm{dBm}$ with frequencies plotted in the figure.

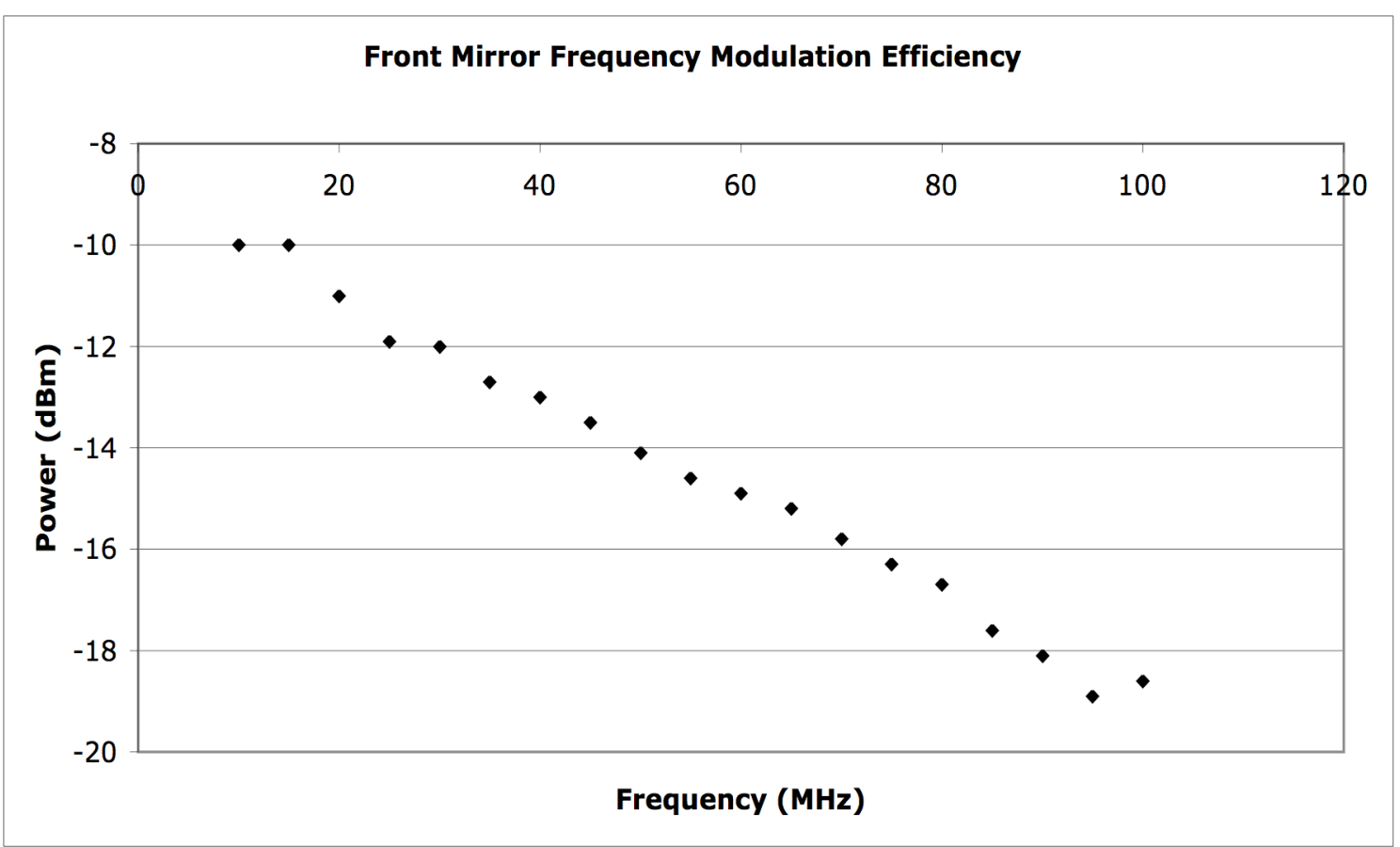

Figure 4.30: Frequency modulation of the front mirror has a $3 \mathrm{~dB}$ drop at 40

MHz. $\mathrm{I}_{\mathrm{SOA}}=150 \mathrm{~mA} ; \mathrm{I}_{\text {gain }}=100 \mathrm{~mA} ; \mathrm{I}_{\text {phase }}=\mathrm{I}_{\mathrm{BM}}=0 \mathrm{~mA} ; \mathrm{I}_{\mathrm{FM}}(\mathrm{DC})=2.1$ $\mathrm{mA}$. $\mathrm{I}_{\mathrm{FM}}(\mathrm{AC})=-70 \mathrm{dBm}$ with frequencies plotted in the figure. 


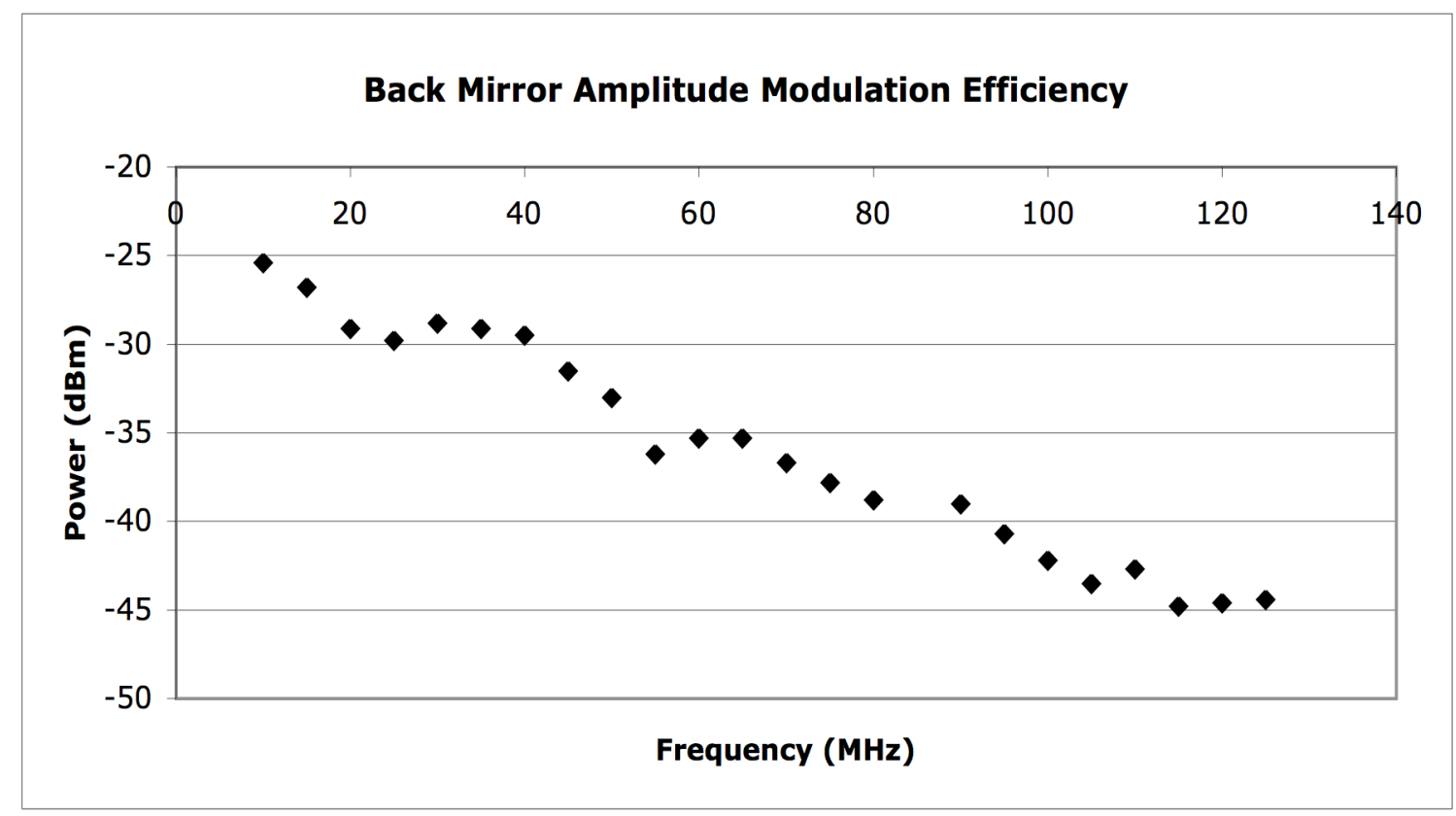

Figure 4.31: Amplitude modulation of the back mirror has a $3 \mathrm{~dB}$ drop at 20 MHz. $\mathrm{I}_{\mathrm{SOA}}=150 \mathrm{~mA} ; \mathrm{I}_{\text {gain }}=100 \mathrm{~mA} ; \mathrm{I}_{\text {phase }}=\mathrm{I}_{\mathrm{FM}}=0 \mathrm{~mA} ; \mathrm{I}_{\mathrm{BM}}(\mathrm{DC})=1.3$ $\mathrm{mA}$. $\mathrm{I}_{\mathrm{BM}}(\mathrm{AC})=-70 \mathrm{dBm}$ with frequencies plotted in the figure.

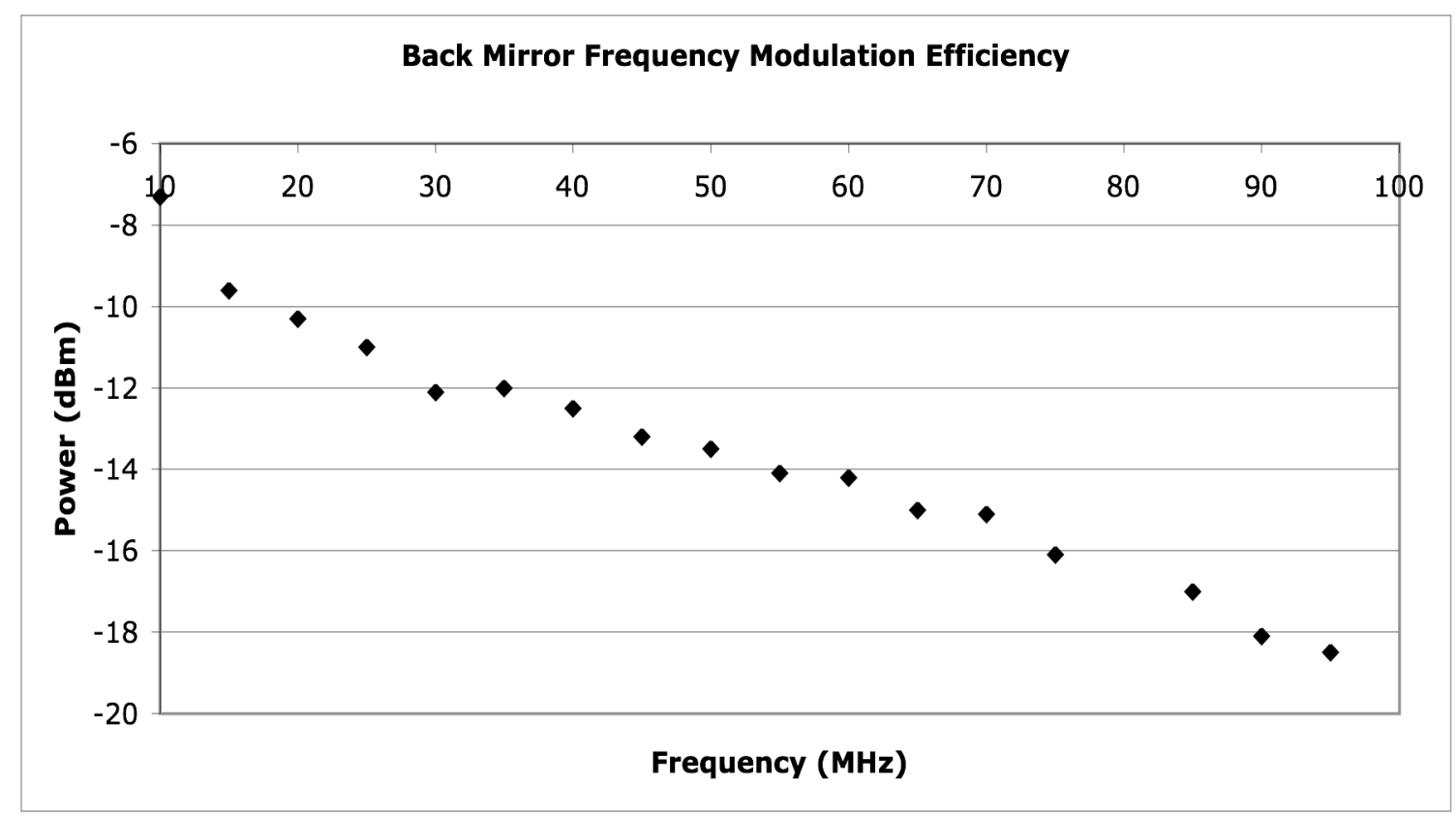

Figure 4.32: Frequency modulation of the back mirror has a $3 \mathrm{~dB}$ drop at $20 \mathrm{MHz}$. $\mathrm{I}_{\mathrm{SOA}}=150 \mathrm{~mA} ; \mathrm{I}_{\text {gain }}=100 \mathrm{~mA} ; \mathrm{I}_{\text {phase }}=\mathrm{I}_{\mathrm{FM}}=0 \mathrm{~mA} ; \mathrm{I}_{\mathrm{BM}}(\mathrm{DC})=1.3 \mathrm{~mA}$. $\mathrm{I}_{\mathrm{BM}}(\mathrm{AC})=-70 \mathrm{dBm}$ with frequencies plotted in the figure. 


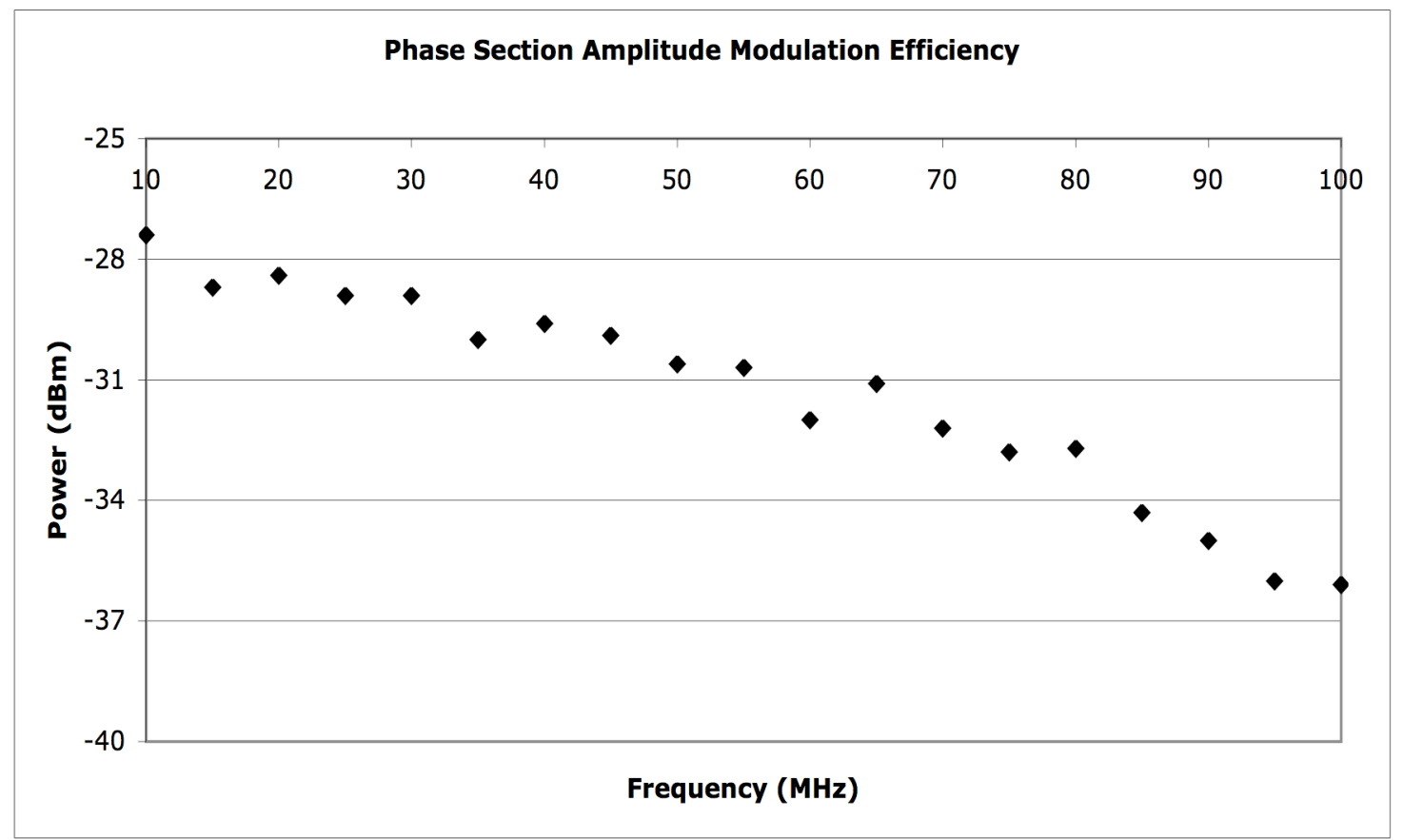

Figure 4.33: Amplitude modulation of the phase section has a $3 \mathrm{~dB}$ drop at 50

MHz. $\mathrm{I}_{\mathrm{SOA}}=150 \mathrm{~mA} ; \mathrm{I}_{\text {gain }}=100 \mathrm{~mA} ; \mathrm{I}_{\mathrm{BM}}=\mathrm{I}_{\mathrm{FM}}=0 \mathrm{~mA} ; \mathrm{I}_{\text {phase }}(\mathrm{DC})=3.4$ $m A$. $I_{\text {phase }}(A C)=-70 \mathrm{dBm}$ with frequencies plotted in the figure.

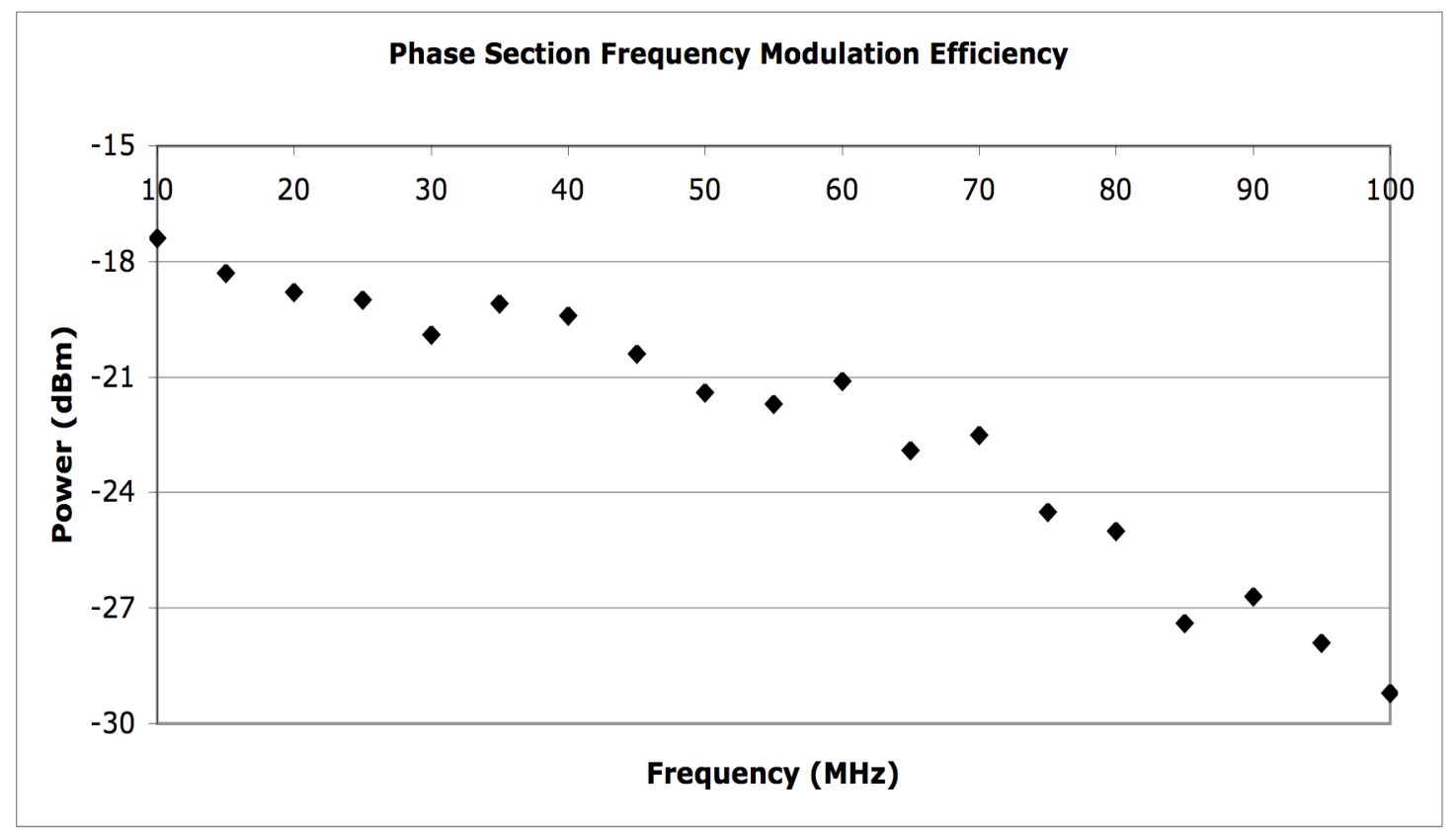

Figure 4.34: Frequency modulation of the phase section has a $3 \mathrm{~dB}$ drop at 45

MHz. $\mathrm{I}_{\mathrm{SOA}}=150 \mathrm{~mA} ; \mathrm{I}_{\text {gain }}=100 \mathrm{~mA} ; \mathrm{I}_{\mathrm{BM}}=\mathrm{I}_{\mathrm{FM}}=0 \mathrm{~mA} ; \mathrm{I}_{\text {phase }}(\mathrm{DC})=3.4$ $\mathrm{mA}$. $\mathrm{I}_{\text {phase }}(\mathrm{AC})=-70 \mathrm{dBm}$ with frequencies plotted in the figure. 


\subsubsection{Modulation Results Summarized}

The results of the modulation measurements are shown in Figures 4.27-4.34. The frequency modulation data is in Appendix A, and the amplitude modulation data is in Appendix E. In each of the figure captions above, the frequency is identified where the power has dropped by $3 \mathrm{~dB}$, or to $50 \%$ of the peak power. This frequency is important because it represents the frequency where the laser section is only varying by half as much as it was at its peak. At this frequency the modulation efficiency is half the value that it is at lower frequencies, but since the input is changing twice in a period, the period represents how quickly the segment can respond to changing input. Therefore, the period of the input modulation signal, found by taking the reciprocal of the modulation frequency, gives the response time of the segment. These numbers are in Table 4.2.

\begin{tabular}{|l|r|r|}
\hline \multicolumn{1}{|c|}{ Input } & $\begin{array}{c}\text { 3 dB Bandwidth } \\
\text { (MHz) }\end{array}$ & \multicolumn{2}{|c|}{$\begin{array}{c}\text { Response Time (ns) } \\
\end{array}$} & 200 & 5 \\
\hline Gain & 200 & 5 \\
\hline SOA & 45 & 22 \\
\hline Phase & 40 & 25 \\
\hline Front Mirror & 20 & 50 \\
\hline Back Mirror & & \\
\hline
\end{tabular}

Table 4.2: Laser segment response times

\subsection{Network Analyzer Input Impedance Measurements}

This next measurement serves to characterize the electrical parasitic limitations of each laser segment as a separate factor from the speed limitations of the internal laser structure. The setup used for this measurement is shown in Figure 4.35. The network analyzer sends out sinusoidal signals at many different frequencies, ranging from $10 \mathrm{MHz}$ to $3 \mathrm{GHz}$. The response to each of these inputs is measured by the network 
analyzer, then the Fourier transform is taken to show the time domain response that would occur to a step input.

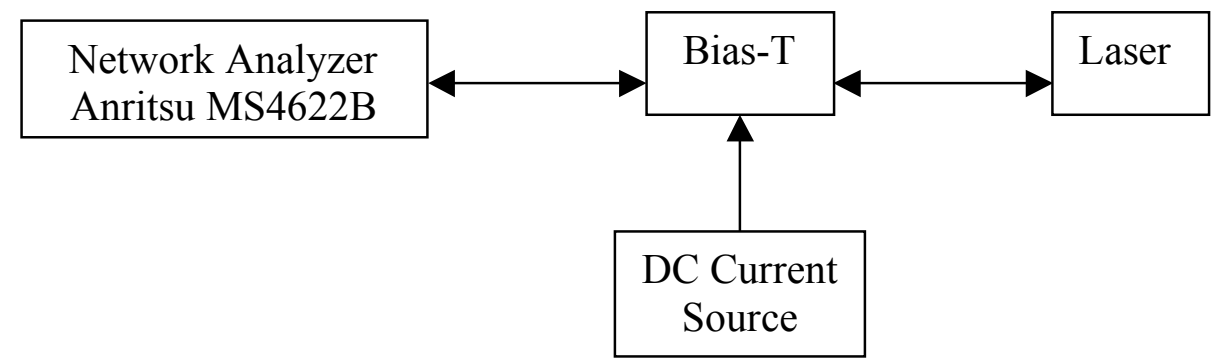

Figure 4.35: Setup for simulating time domain responses from the laser inputs.

A proposed circuit model for the input impedance of the package lasers shown in Figure 4.3 (p. 52) is shown in Figure 4.36. The reference plane for this model is at the end of the $.085 "$ semi-rigid coax connections. The inductance is due to the butterfly lead connecting the mini-coax cable to the inside of the laser and wire bonds within the package. The laser segments can be represented as a diode, which at a single bias point can be represented as a capacitor and resistor in parallel. The capacitor represents the diffusion capacitance of the laser segment and the resistor represents the dynamic resistance of the forward biased diode.

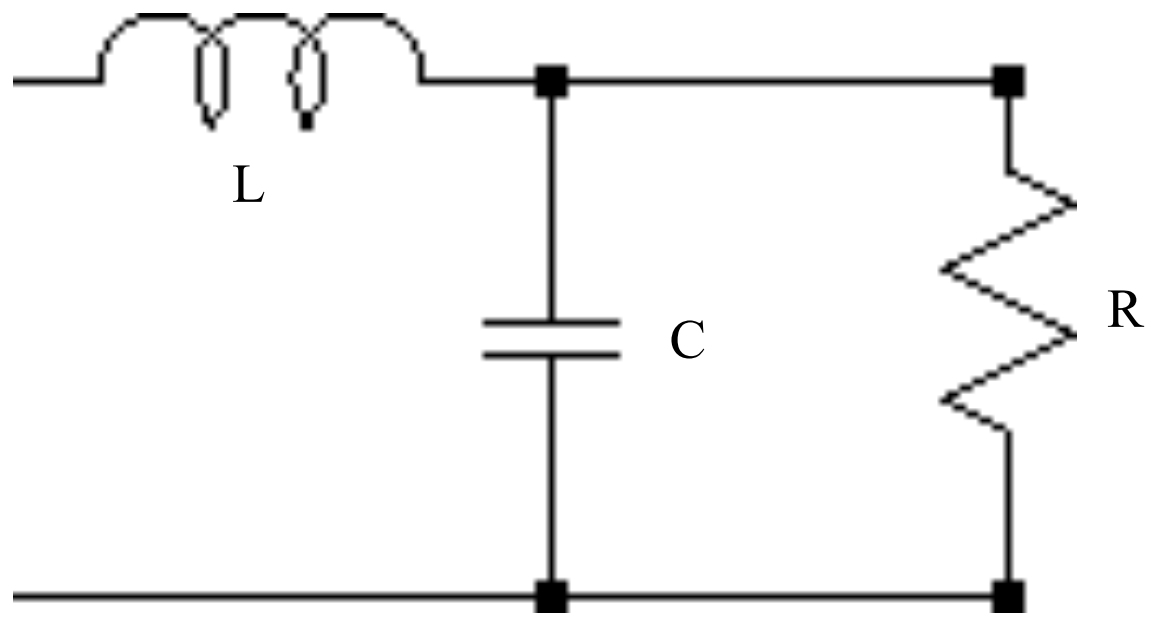

Figure 4.36: Circuit model of the laser inputs. 


\section{Phase Segment Time Domain Response}

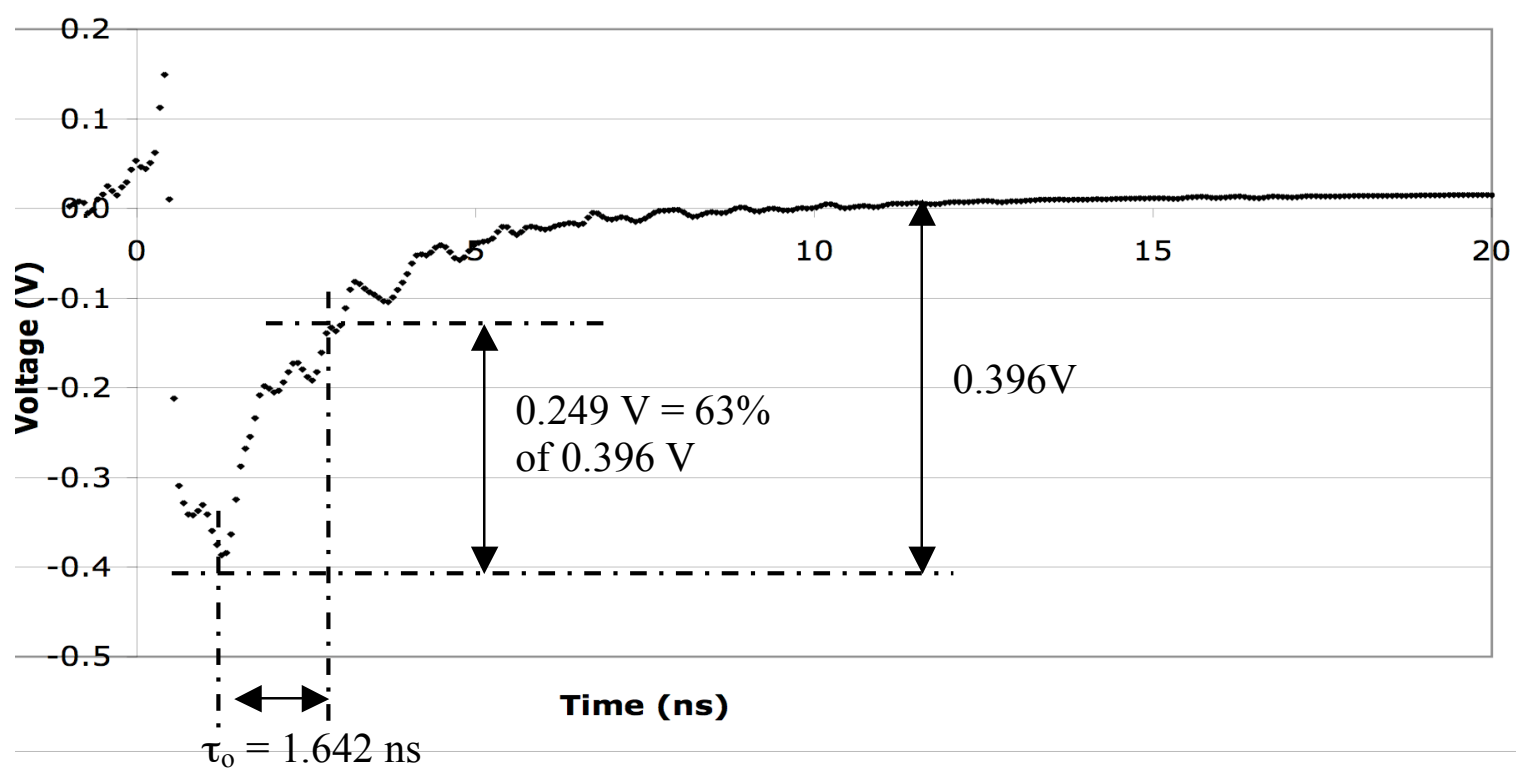

Figure 4.37: Time domain response, phase segment. $I_{\text {phase }}(\mathrm{DC})=3.4 \mathrm{~mA}$. The difference between the minimum voltage and the steady-state voltage is shown. Also shown is the time needed for the signal to rise $63 \%$ of the way from its minimum to its final value.

Figure 4.37 shows the time domain response given by the network analyzer for the phase segment. There is an initial voltage spike due to the inductance. After that there is an exponential decay as the voltage increases. The exponential decay can be used to find the $\mathrm{RC}$ time constant and the bandwidth of the input due to the diode. The time constant is measured from the lowest value until the voltage rises $63 \%$ of the way to its final value. The rise time $\tau_{\mathrm{o}}=1.642 \mathrm{~ns}$ gives a $3 \mathrm{~dB}$ bandwidth of $1 /\left(2 * \pi^{*} \tau_{\mathrm{o}}\right)=96.9 \mathrm{MHz}$ 
Front Mirror Time Domain Response

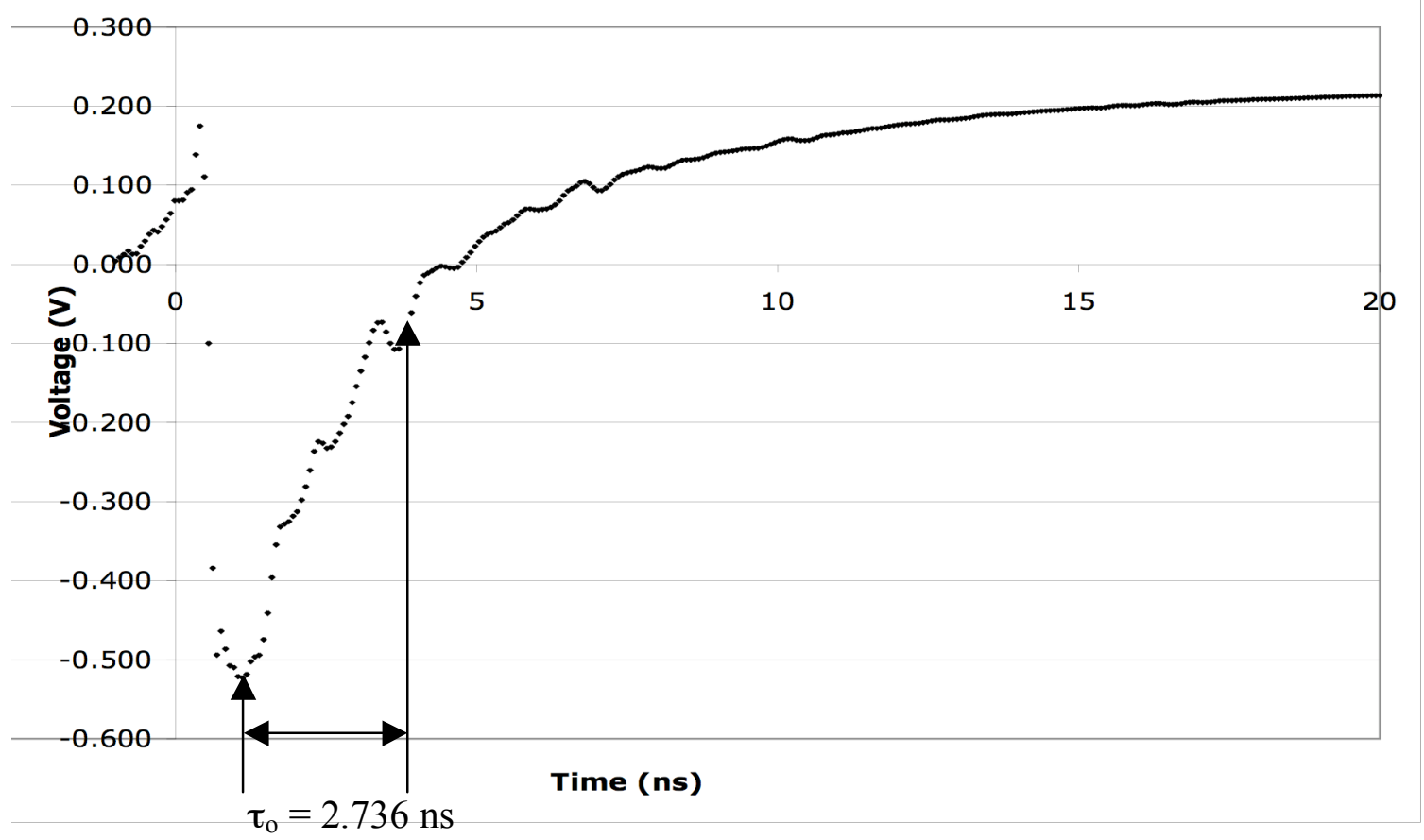

Figure 4.38: Time domain response, front mirror segment. $\mathrm{I}_{\mathrm{FM}}(\mathrm{DC})=2.1 \mathrm{~mA}$.

Figure 4.38 shows the time domain response for the front mirror segment. In this case, $\tau_{\mathrm{o}}=2.736 \mathrm{~ns}$, which gives a $3 \mathrm{~dB}$ bandwidth of $1 /\left(2 * \pi^{*} \tau_{\mathrm{o}}\right)=58.2 \mathrm{MHz}$. 


\section{Back Mirror Time Domain Response}

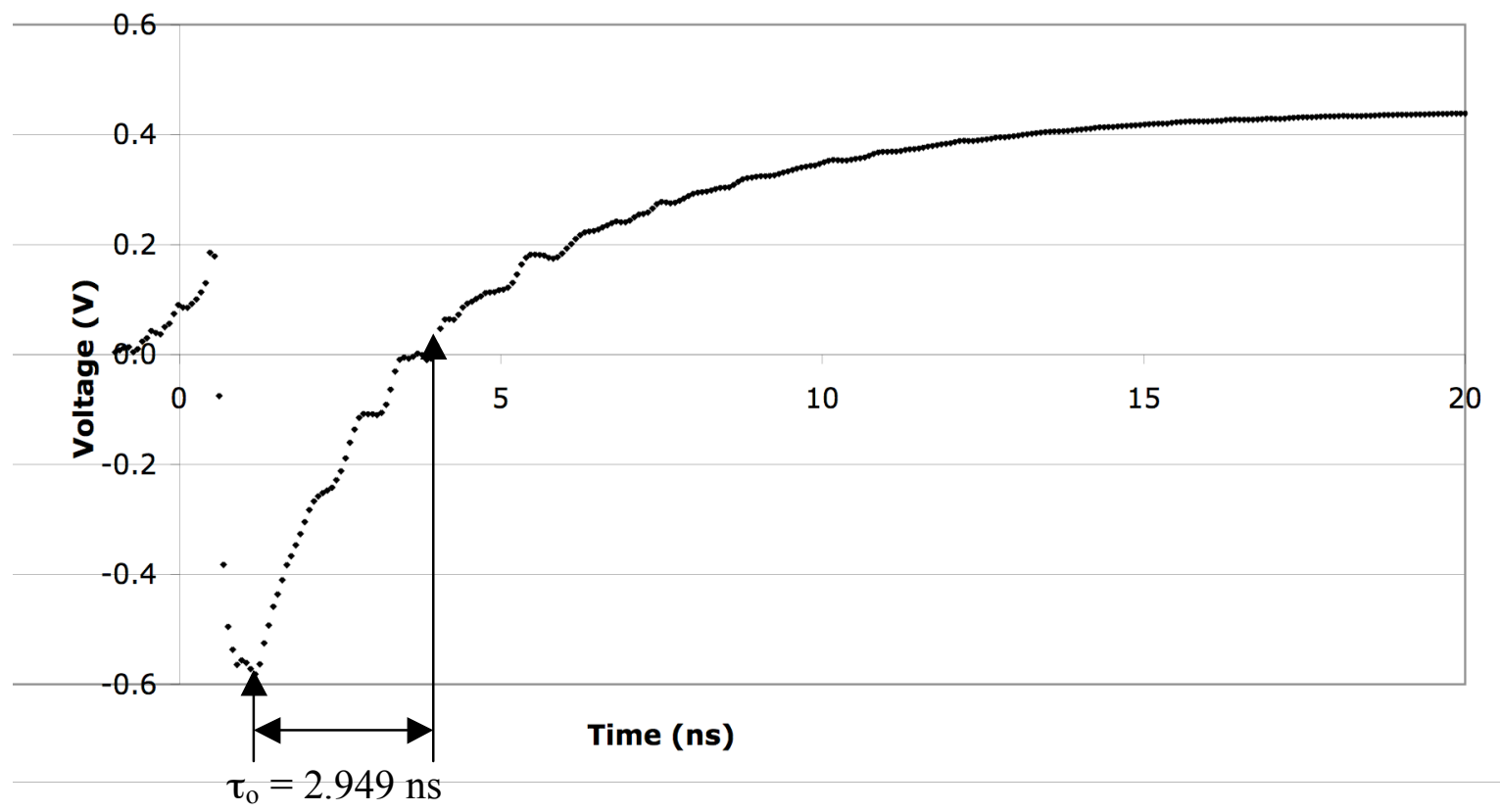

Figure 4.39: Time domain response, back mirror segment. $\mathrm{I}_{\mathrm{BM}} \mathrm{DC}=1.3 \mathrm{~mA}$.

Figure 4.39 shows the time domain response for the front mirror segment. In this case, $\tau_{\mathrm{o}}=2.949 \mathrm{~ns}$, which gives a $3 \mathrm{~dB}$ bandwidth of $1 /\left(2 * \pi^{*} \tau_{\mathrm{o}}\right)=54 \mathrm{MHz}$. 


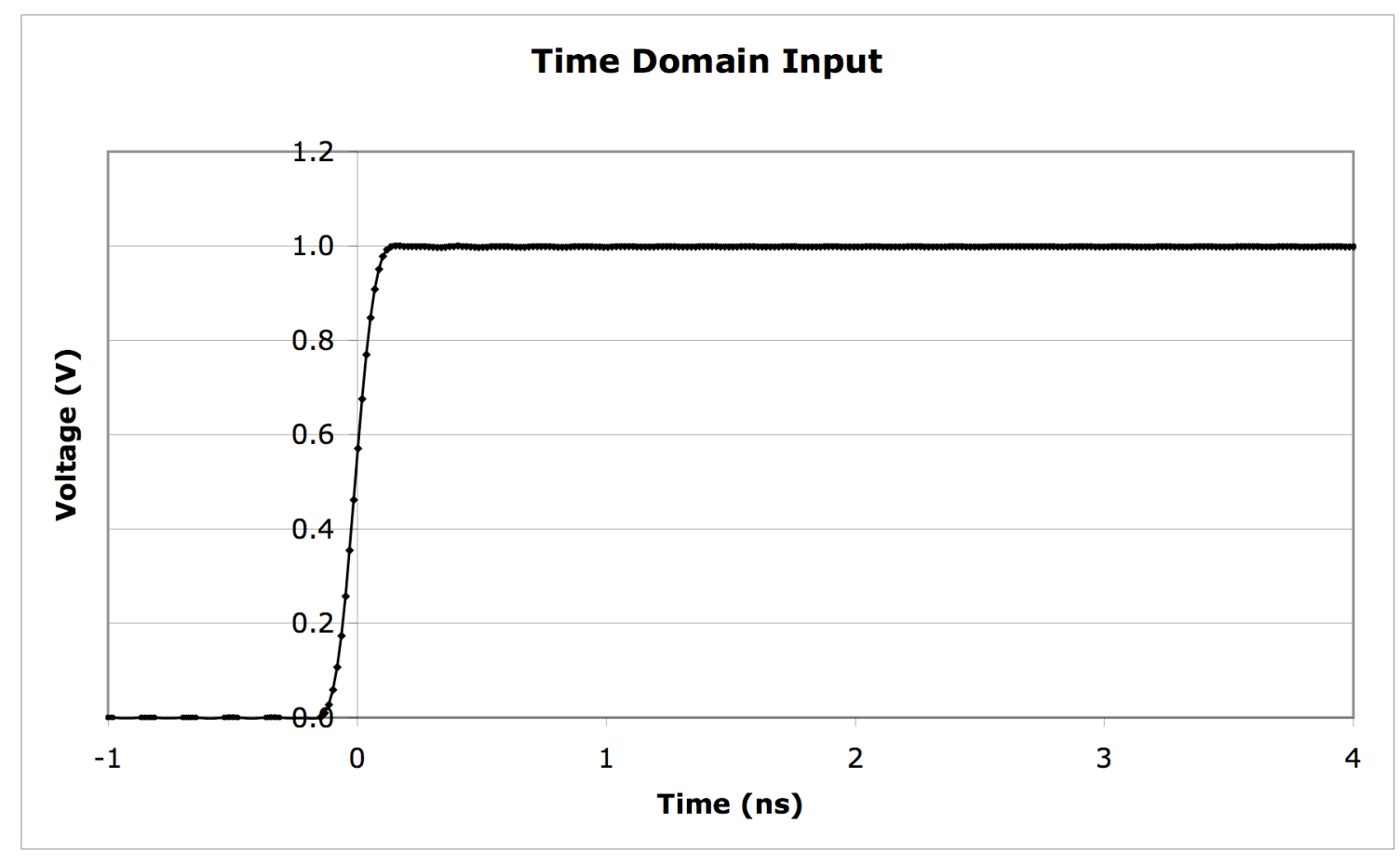

Figure 4.40: Input into the laser segments. This was measured with an open circuit at the reference plane. The $10 \%$ to $90 \%$ rise time is $0.15 \mathrm{~ns}$.

\begin{tabular}{|l|r|r||r|}
\hline Input segment & $\tau_{\mathbf{o}}(\mathbf{n s})$ & $\begin{array}{c}\text { Time Domain } \\
\text { Bandwidth (MHz) }\end{array}$ & $\begin{array}{c}\text { Modulation Bandwidth } \\
\text { Measurement (MHz) }\end{array}$ \\
\hline Phase & 1.642 & 96.9 & 45 \\
\hline Front Mirror & 2.736 & 58.2 & 40 \\
\hline Back Mirror & 2.949 & 54.0 & 20 \\
\hline
\end{tabular}

Table 4.3: Summary of time domain responses for different laser inputs. The Time Domain Bandwidth is calculated as $1 /\left(2 * \pi * \tau_{\mathbf{o}}\right)$ from the measurements in this section. The Modulation Bandwidth Measurement column is copied from Table 4.2 for comparison.

The Time Domain Bandwidth figures in Table 4.3 are larger than what was found from amplitude and frequency modulation measurements (repeated in Table 4.3 for comparison). Since the time domain bandwidth values only look at the RC delay and don't take the initial spike into account, an error on the side of a larger bandwidth is expected. 


\begin{tabular}{|l|r|r|r|}
\hline Segment & L (nH) & $\mathbf{C ~ ( p F ) ~}$ & R ( $\mathbf{\Omega})$ \\
\hline Phase & 11 & 12 & 52 \\
\hline Front Mirror & 14 & 30 & 80 \\
\hline Back Mirror & 13 & 40 & 125 \\
\hline
\end{tabular}

Table 4.4: Estimated circuit values for the laser inputs, using the circuit model from Figure 4.36 (p. 74).

Using the time domain responses, circuit models were created to approximate the laser inputs. Values were put into MicroCap for the three circuit components, and they were changed until the simulated time domain response matched fairly closely with the measured time domain response. The values that provided matching responses are listed in Table 4.4.

Based on these measurements, the bandwidth limitations for laser operation are based on electrical connections to the laser rather than limitations of the internal laser operation. These measurements don't rule out the laser operation as a limiting factor, but they demonstrate that the laser inputs have a limited bandwidth. This is likely caused by the diode capacitance. A setup starting with a chip laser, such as the setup attempted in Chapter 3, should have a larger bandwidth.

\subsection{Linewidth Measurements}

\subsubsection{Experimental Setup}

One of the important parameters for a laser is its linewidth. Ideally a laser would operate at a single wavelength, but in reality there is some spread to the wavelength that comes from the laser. Since this spread in wavelength can affect the accuracy of any measurements made with the laser, it is important to characterize it. One way of 
representing this value is with the full-width half maximum (FWHM) frequency, which is the difference between the half-power frequencies around the maximum frequency.

Linewidth is an important parameter for OCT measurements because a spectrum analyzer ultimately has to resolve the frequency of the signal. A larger linewidth decreases the accuracy of this frequency determination and limits the accuracy of OCT measurements.

\subsubsection{Theory}

The technique used to make this measurement is called the delayed selfhomodyne technique. The basic principle for this technique is that, if an optical signal is combined with a delayed version of itself, and the delay time is larger than the coherence time for the optical signal, then it will be as if two different optical signals were combined that are operating at the same frequency but with no phase relationship.

$$
S_{i}(f) \approx \mathfrak{R}^{2}\left\{S_{d}(f)+2\left[S_{S}(f) \otimes S_{S}(-f)\right]\right\}
$$

As shown in Equation 4.1, the resulting signal has a direct detection term, $\mathrm{S}_{\mathrm{d}}(\mathrm{f})$, and a mixing term of $\mathrm{S}_{\mathrm{S}}(\mathrm{f})$ (the laser output) being convoluted with itself. $\Re$ is the responsivity of the photodetector, which has units of A/W. For the purpose of measuring linewidth, only the mixing term is important. Figure 4.41 shows the effect of this convolution on the laser signal and how it gives a low-frequency signal where the linewidth can be measured. 
a)

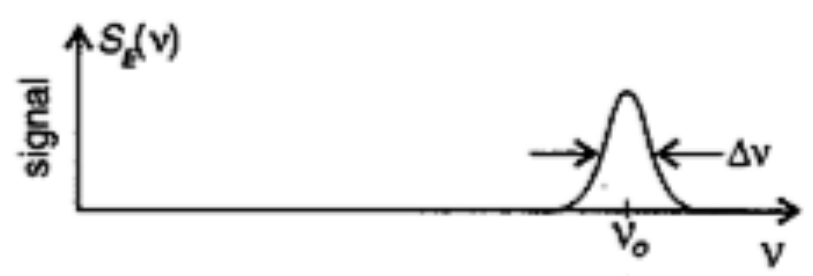

b)

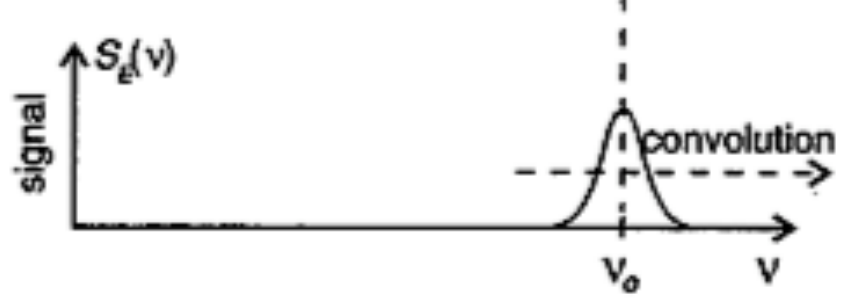

c)

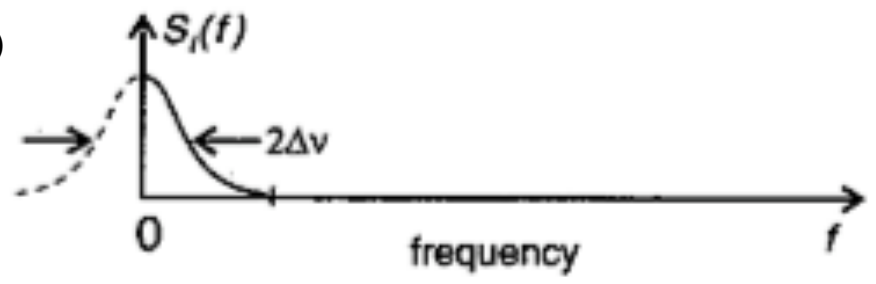

Figure 4.41: The process of combining a signal with itself to get a signal where the linewidth can be measured [3]. Part a) is a spectral plot of the laser output, and $\Delta v$ indicates the linewidth. Part b) is the same laser output, except it has been delayed so that it doesn't have any phase relationship with a). In part c) the laser output has been convolved with itself to form a wider signal around DC.

For a Lorentzian signal, the convolution doubles the width of the signal and centers it around $0 \mathrm{~Hz}$. Half of the signal isn't displayed on the Electrical Spectrum Analyzer (ESA) because it is below $0 \mathrm{~Hz}$, so the frequency where the power has dropped by $3 \mathrm{~dB}$ is the FWHM linewidth of the laser.

\subsubsection{Setup}

To implement the delayed self-homodyne setup, a Mach-Zehnder interferometer was used. The recombined light is absorbed by a photodiode. This signal is then sent into an amplifier before going to the ESA [3]. This is shown in Figure 4.42. 
The interferometer used for this setup is not the same one used for making frequency modulation measurements. An important part of making linewidth measurements using the delayed self-homodyne method is ensuring that the delay time from the interferometer is larger than the coherence length from the laser light. To avoid error, the condition $\Delta f^{*} \tau_{\mathrm{o}} \geq 1$ needs to be satisfied, where $\Delta f$ is the linewidth and $\tau_{\mathrm{o}}$ is the delay time [3]. For the setup measuring linewidth, the interferometer provided a delay time of $3.5 \mu \mathrm{s}$. This allows linewidths as small as $286 \mathrm{kHz}$ to be measured. This condition needs to be checked after linewidth measurements have been made.

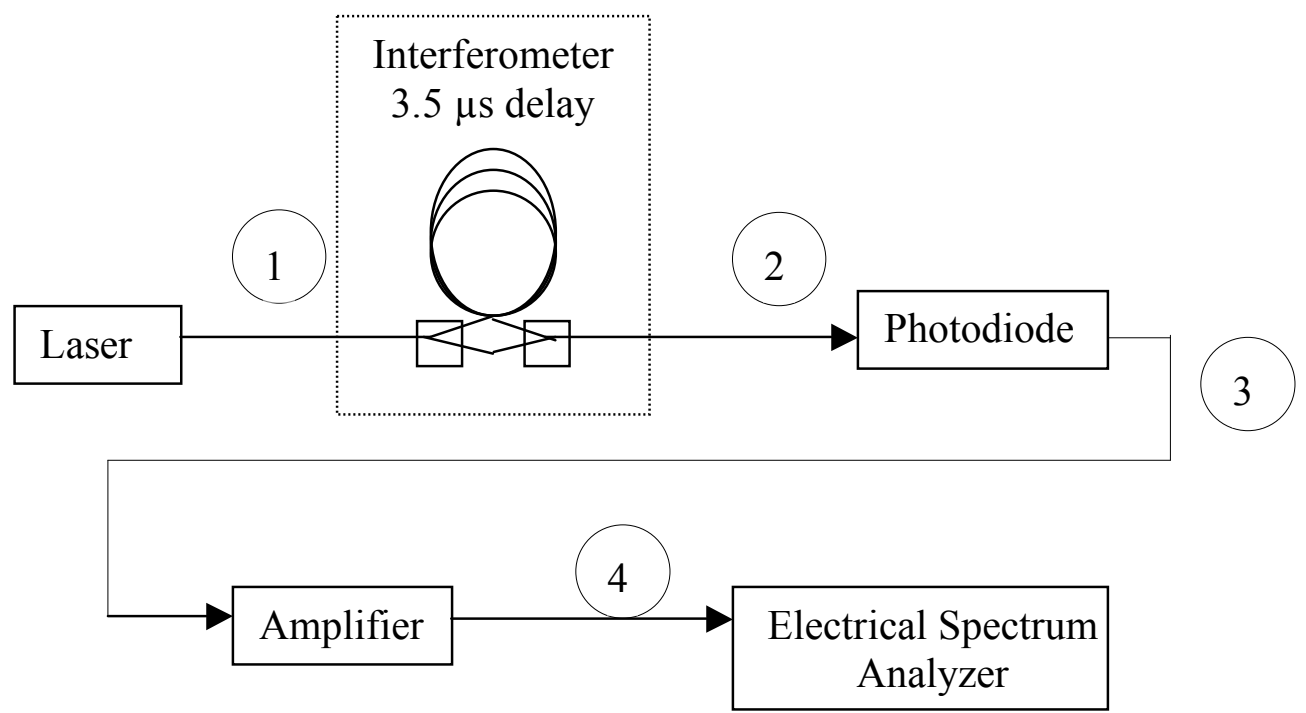

Figure 4.42: Block diagram for measuring linewidth. (1) Output from the laser (optical signal). (2) Laser output combined with a delayed version of itself (optical signal). (3) Difference frequency between the two (nearly identical) optical signals, centered around DC (electrical signal). (4) Amplified signal ( $+40 \mathrm{~dB})$ (electrical signal).

The data was collected by using a custom written LabView file to read all of the points off of the ESA into a text file. Then the data was copied into Excel to create the plot. There are several plots of this measurement starting with Figure 4.43. There is a 
peak right at the beginning due to local oscillator feedthrough. There is a dip after this because the amplifier used had a lower corner frequency of $8 \mathrm{MHz}$, as shown in Figure 4.17 (p. 60).

The value for linewidth is determined by getting the peak power measurement and then finding the frequency where the power has dropped by $3 \mathrm{~dB}$ [3]. All of this assumes that the laser has a Lorentzian distribution. This assumption was not tested, but a Lorentzian distribution is common for lasers.

Due to the amplifier not properly covering the range below $8 \mathrm{MHz}$, the measured $3 \mathrm{~dB}$ frequency will be greater than the actual FWHM linewidth. The error in the $f_{3 \mathrm{~dB}}$ point should be less than $8 \mathrm{MHz}$. The error can be reduced by measuring a frequency where a larger power drop occurs and adjusting the measurement accordingly. In general, the correction factor is found by finding the ratio between the peak power and the power at that frequency (in terms of absolute power), subtracting 1, taking the square root, and dividing the frequency by that value.

For example, if the power has dropped by $10 \mathrm{~dB}$ at $50 \mathrm{MHz}$, then the ratio of the peak power to the power at that frequency is $10 / 1$, so the actual FWHM frequency is $50 / \sqrt{10-1}$, or $50 / \sqrt{9}$, or $50 / 3 \mathrm{MHz}$. As another example to make sure this fits the basic definition, if a drop of $3 \mathrm{~dB}$ occurs at $35 \mathrm{MHz}$, then the ratio of the peak power to that power level is $2 / 1$, which means the actual FWHM frequency is $35 / \sqrt{2-1}$, or simply $35 \mathrm{MHz}$, which is expected based on the definition of the FWHM frequency.

Most of the linewidth measurements show a drop of at least $8 \mathrm{~dB}$, so this is the value used for comparison between lasers. This means that the power ratio is 6.31 , so the frequency where the power has dropped by $8 \mathrm{~dB}$ is divided by $\sqrt{5.31}$. 


\subsubsection{Results}

The linewidth was measured for several lasers. This is done to compare the JDSU laser and the setup built for it with other lasers that are available. A narrow linewidth is needed to use a laser for OCT measurements, so it is necessary that the JDSU laser meets this criterion. Comparing the linewidth between several lasers allows a determination of how narrow the linewidth is.

\subsubsection{Fixed Wavelength DFB Laser Linewidth}

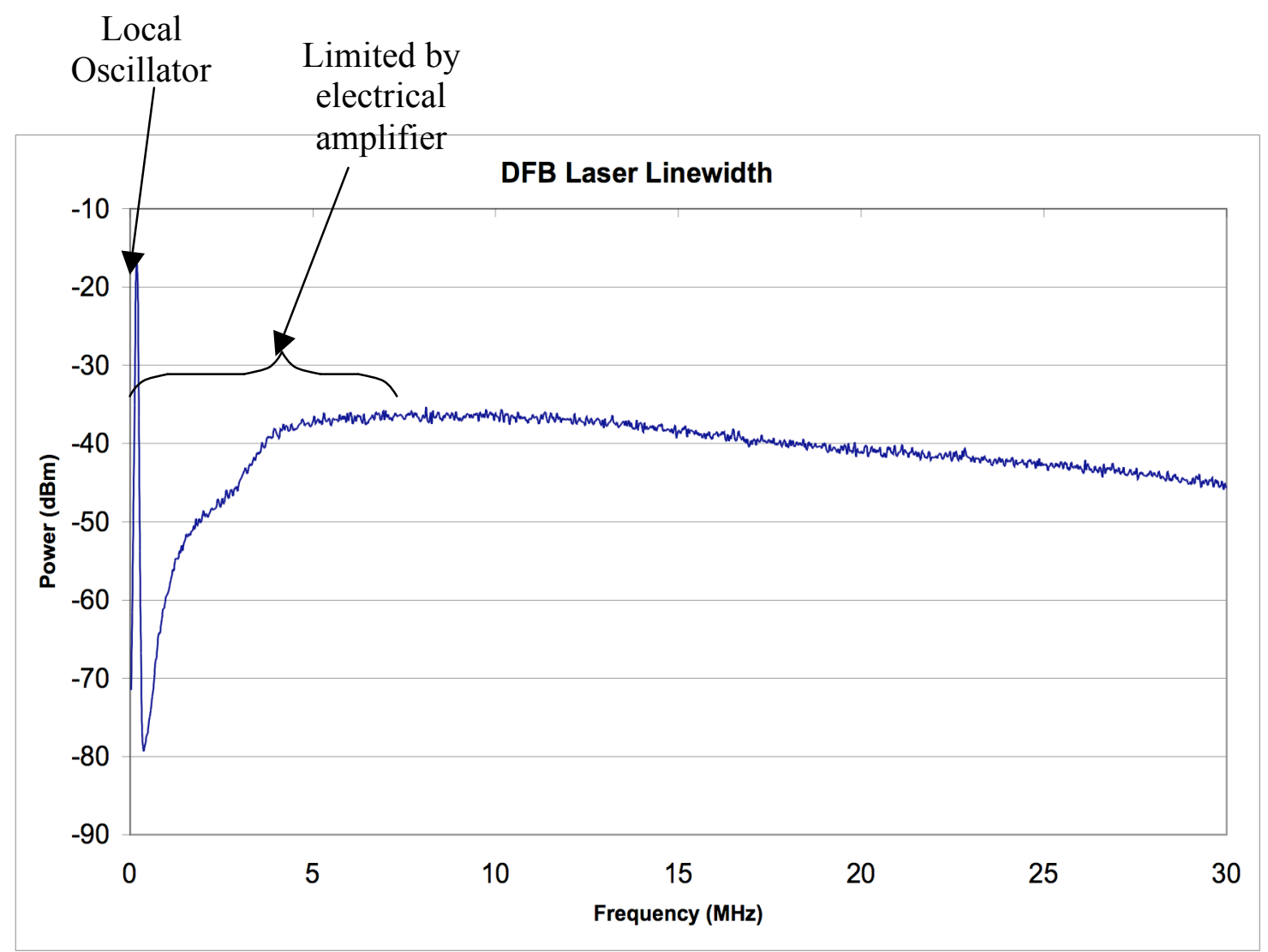

Figure 4.43: Linewidth measurement for the DFB laser 
The first laser measured was a fixed wavelength distributed feedback (DFB) laser. This is a laser that is similar to what could be used in a telecommunications application. The plot for that is shown in Figure 4.43.

\subsubsection{Nortel Tunable Laser (VCSEL) Linewidth}

Another laser that was measured for comparison is a vertical cavity surfaceemitting laser (VCSEL) made by Nortel. This is another laser that finds use in telecommunications applications. Five different linewidth measurements were done with this laser at different wavelengths, and those values are recorded in Table 4.5.

\begin{tabular}{|r|r|r|}
\hline Input (V) & Wavelength (nm) & -3 dB Linewidth (MHz) \\
\hline 5 & 1576.3 & 24.2 \\
\hline 10 & 1574.3 & 27.0 \\
\hline 20 & 1565.8 & 26.2 \\
\hline 25 & 1558.9 & 28.6 \\
\hline 40 & 1518.0 & 24.5 \\
\hline
\end{tabular}

Table 4.5: Linewidth measurements for the Nortel tunable laser.

\subsubsection{Bookham Laser Linewidth}

The third laser measured for comparison was the LambdaFLEX iTLA laser made by Bookham. This laser is also a SGDBR laser, and it has its own software for controlling the wavelength from the computer. Figure 4.44 shows a plot of this linewidth measurement. 


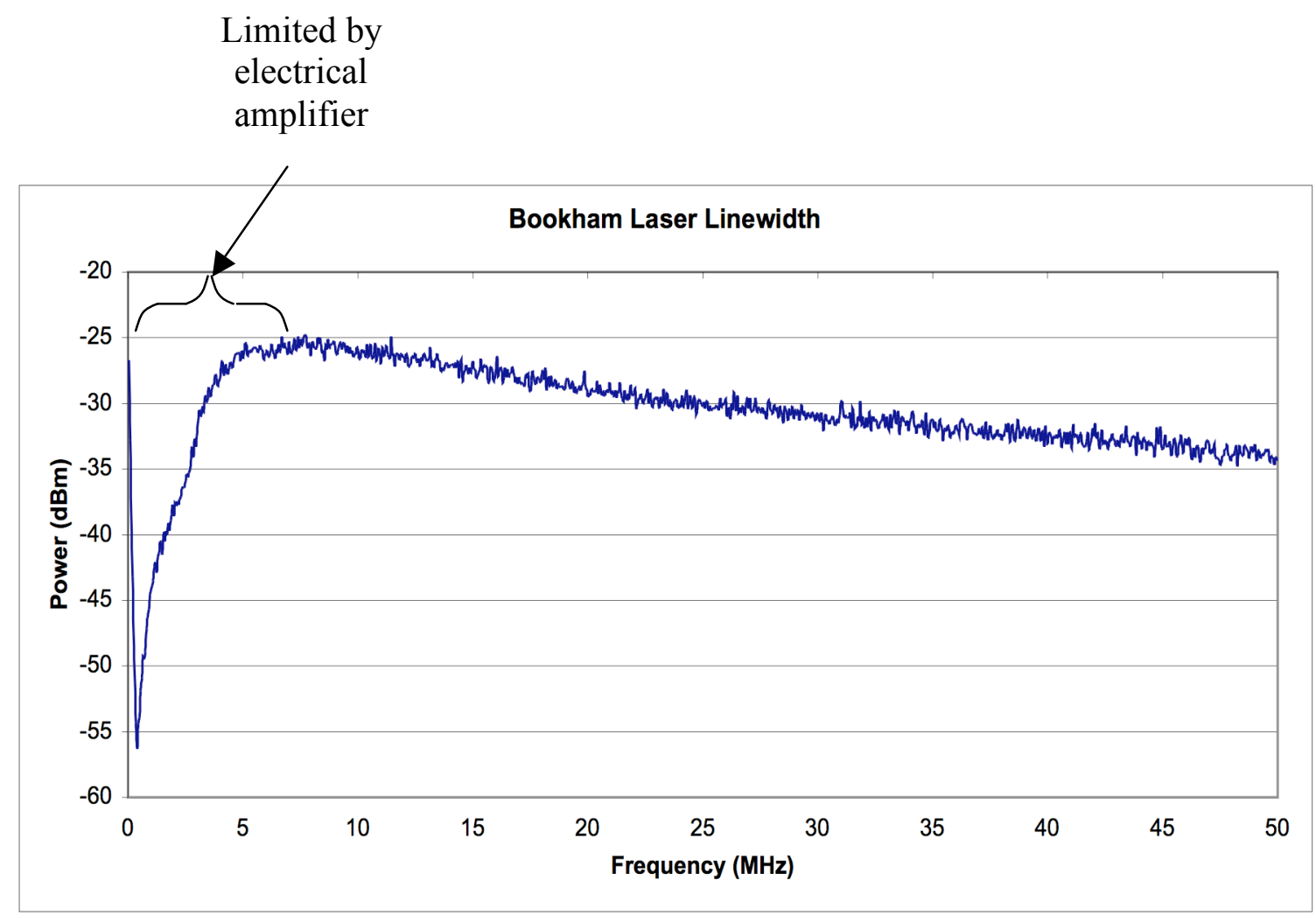

Figure 4.44: Linewidth measurement for the Bookham laser.

\subsubsection{JDSU Laser Linewidth}

The MKS-063-B laser provided by JDSU is the laser that was used for the rest of the measurements in this paper. Multiple linewidth measurements were made for the JDSU packaged laser for different input values, with only one wavelength-controlling segment receiving current for each measurement. The input values match what was used for later measurements. Those are shown in Figures 4.45-4.47. 


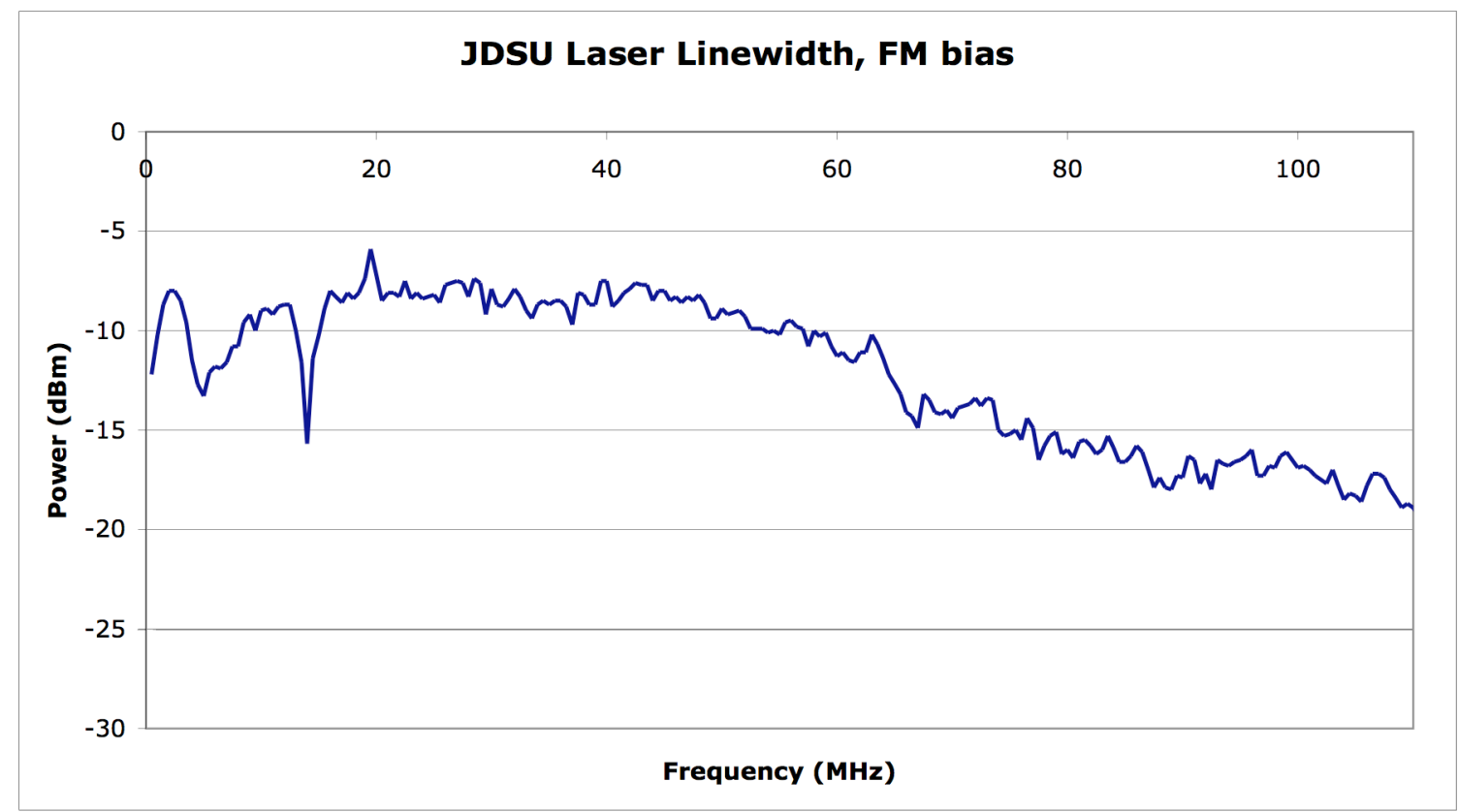

Figure 4.45: Linewidth measurement for the JDSU laser, front mirror bias. $\mathrm{I}_{\text {gain }}=100 \mathrm{~mA}, \mathrm{I}_{\mathrm{SOA}}=150 \mathrm{~mA}, \mathrm{I}_{\mathrm{BM}}=\mathrm{I}_{\text {phase }}=0 \mathrm{~mA}, \mathrm{I}_{\mathrm{FM}}=2.1 \mathrm{~mA}$.

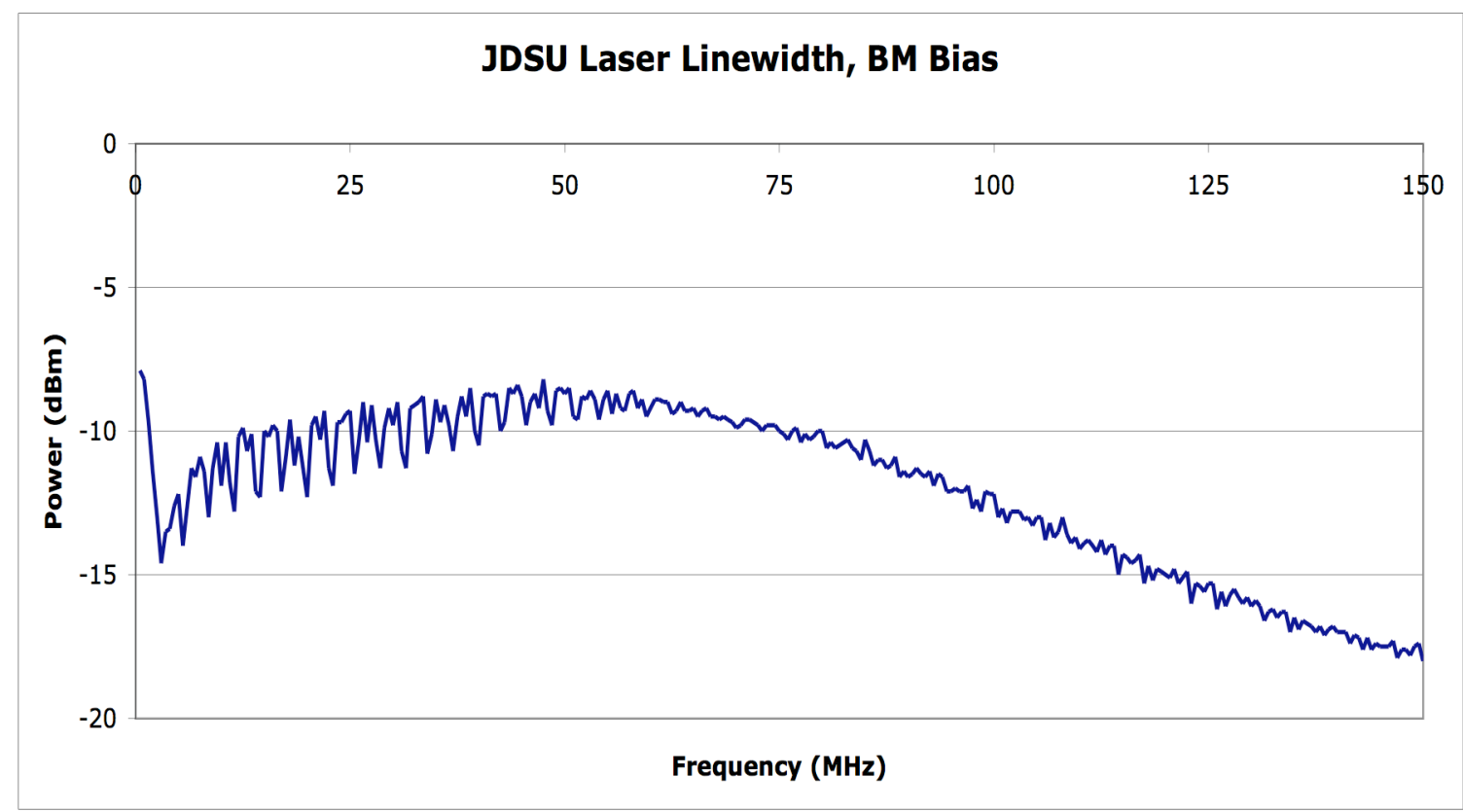

Figure 4.46: Linewidth measurement for the JDSU laser, back mirror bias. $\mathrm{I}_{\text {gain }}=100 \mathrm{~mA}, \mathrm{I}_{\mathrm{SOA}}=150 \mathrm{~mA}, \mathrm{I}_{\mathrm{FM}}=\mathrm{I}_{\text {phase }}=0 \mathrm{~mA}, \mathrm{I}_{\mathrm{BM}}=1.3 \mathrm{~mA}$. 


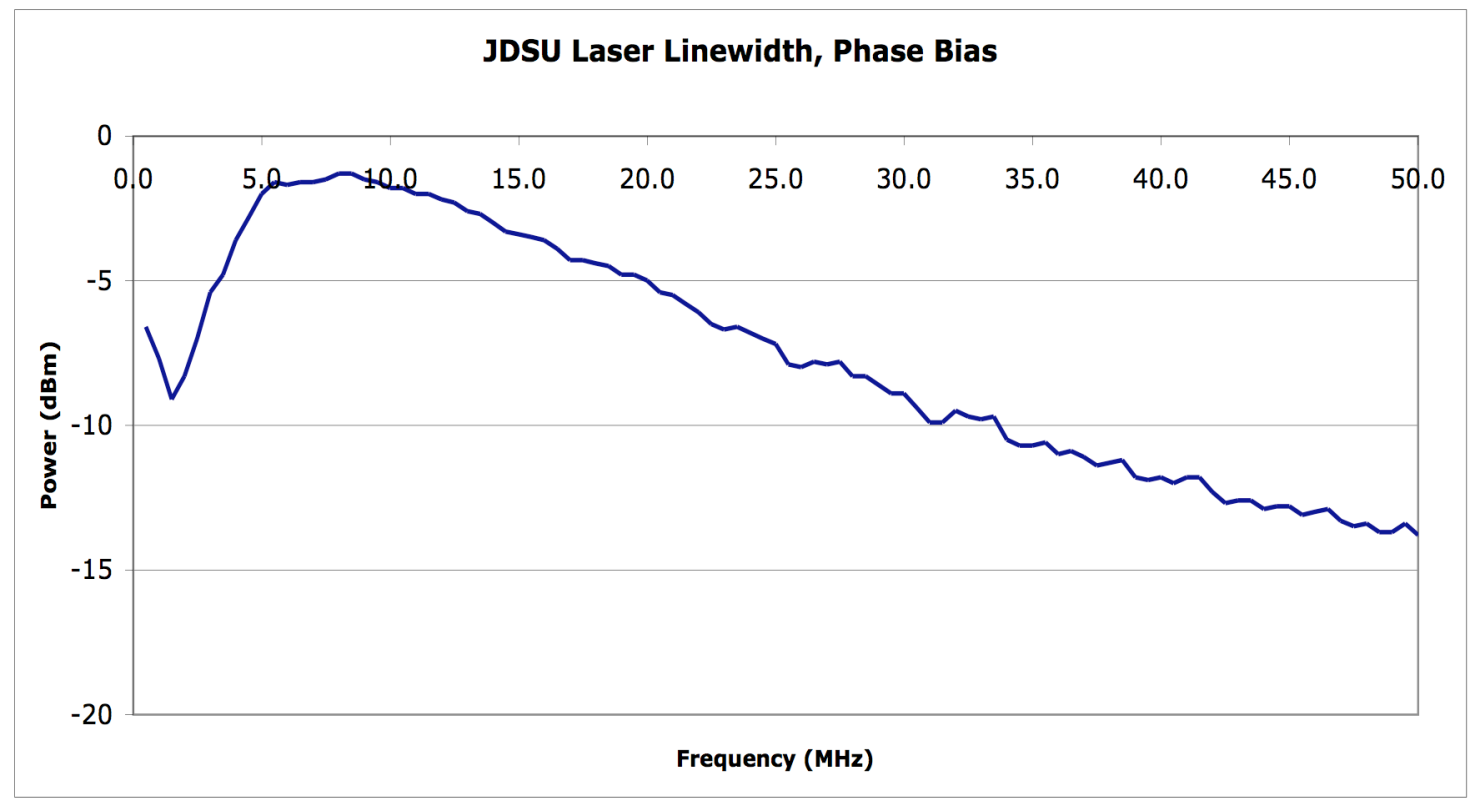

Figure 4.47: Linewidth measurement for the JDSU laser, phase bias. $\mathrm{I}_{\text {gain }}=100 \mathrm{~mA}, \mathrm{I}_{\mathrm{SOA}}=150 \mathrm{~mA}, \mathrm{I}_{\mathrm{BM}}=\mathrm{I}_{\mathrm{FM}}=0 \mathrm{~mA}, \mathrm{I}_{\text {phase }}=3.1 \mathrm{~mA}$.

\subsubsection{Summary of Linewidth Measurements}

The measurements are combined into Table 4.6. The linewidths for all of the comparison lasers are similar to each other. For the JDSU laser, when a DC bias is applied to the phase section, the wavelength is similar to that of the other lasers. When a DC bias is applied to the front mirror or back mirror, the linewidth is several times larger. Since the JDSU laser has a reasonable linewidth compared to other lasers, it should be usable in OCT measurements.

\begin{tabular}{|l|r|r|r|}
\hline \multicolumn{1}{|c|}{ Laser } & \multicolumn{1}{c|}{$\begin{array}{c}\text { 3 dB Linewidth } \\
\text { (MHz) }\end{array}$} & \multicolumn{1}{c|}{$\begin{array}{c}\text { 8 dB Linewidth } \\
\text { (MHz) }\end{array}$} & $\begin{array}{c}\text { Equivalent 3 dB } \\
\text { Linewidth (MHz) }\end{array}$ \\
\hline DFB & 17.4 & 28.9 & 12.5 \\
\hline Nortel & 26.1 & N/A & N/A \\
\hline Bookham & 19.0 & 48.0 & 20.8 \\
\hline JDSU BM bias & 94.0 & 137.0 & 59.5 \\
\hline JDSU FM bias & 59.5 & 86.5 & 37.5 \\
\hline JDSU Phase bias & 19.5 & 33.5 & 14.5 \\
\hline
\end{tabular}

Table 4.6: Summary of linewidth measurements. The Equivalent $3 \mathrm{~dB}$ Linewidth column is created by dividing the values in the $8 \mathrm{~dB}$ Linewidth column by $\sqrt{5.31}$. 


\subsection{Summary of Results}

This chapter outlined the process used to construct the package for the laser provided to Cal Poly by JDSU. Connections were made to the butterfly leads with added capacitance to compensate for the inductance of the leads. Semi-rigid cables were used to make the connections off of the laser and out to the BNC cables.

DC operation of the laser was characterized. This verified that all of the laser segments worked as expected. This process showed how the laser changes in wavelength and power when an input is changed. Some mode switches were identified to avoid for later measurements.

Setups to measure amplitude and frequency modulation were created. Bias points were identified to effectively measure frequency modulation. These bias points were used to determine the response times of each laser segment. All of the laser segments can be switched at $50 \mathrm{~ns}$ or faster, verifying the fast wavelength switching of the laser.

Time-domain measurements were done to measure the response of the laser inputs independent from the laser's operation. These measurements identified an upper bound for the bandwidth of the inputs. This measurement helps verify the results from the amplitude and frequency modulation measurements. It also identifies the electrical connection as the limiting factor, rather than the actual laser.

The linewidth for this laser was measured. Linewidth for other similar lasers was measured to compare with the JDSU laser. Under some bias conditions the JDSU laser is as good as other lasers, and in other cases it is a little bit worse. In all cases it is acceptable for OCT measurements.

The basic values for characterizing the laser are in Table 4.7. 


\begin{tabular}{|l|r|r|}
\hline \multicolumn{1}{|c|}{ Segment } & \multicolumn{1}{|c|}{$\begin{array}{c}\text { 3 dB Response } \\
\text { Time (ns) }\end{array}$} & $\begin{array}{c}\text { Linewidth } \\
\text { (MHz) }\end{array}$ \\
\hline SOA & 5 & N/A \\
\hline Gain & 5 & N/A \\
\hline Front Mirror & 25 & 37.5 \\
\hline Back Mirror & 50 & 59.5 \\
\hline Phase & 22 & 14.5 \\
\hline
\end{tabular}

Table 4.7: Summary of measurements characterizing laser inputs 


\section{CHAPTER 5: Conclusion}

This thesis showed that fast wavelength switching can be achieved using SGDBR lasers. High-speed wavelength switching is an important property for future research in OCT applications of SGDBR lasers.

Chapter 2 explored a potential application for OCT measurements in determining meat tenderness. Though no correlation was found to allow OCT measurements to be used for meat tenderness testing, there is still the possibility that other methods of analysis could identify a connection in the data. More applicable is the demonstration of how OCT measurements can be useful in general, since some applications will be able to make use of the data collected.

Furthermore, the slow measurement speed was an obstacle in collecting the OCT measurements for the meat data. Each measurement took about a minute to collect when everything was going smoothly, which would be a large bottleneck to a process like this. Faster measurement techniques would reduce this bottleneck.

Chapter 3 examined the properties of chip lasers from UC Santa Barbara. These lasers looked promising in some ways, such as being able to tune the wavelength by varying the phase input current. Eventually it was determined that light could not be focused into a lens system because it did not have a single focal point. A few measurements were made to show that the laser output for the chip laser can be controlled. This led to experimentation with a packaged laser.

In Chapter 4 the properties of a packaged laser from JDSU were explored. This laser was provided with internal capacitance ranging from 12 to $40 \mathrm{pF}$. A setup was 
constructed to provide current to the different segments while controlling impedance to the high speed pins. Basic DC functionality of amplitude and frequency tuning on each of the lasers segment was measured.

It was demonstrated that fast wavelength switching can be obtained with this laser for use in OCT applications. High speed AM and FM modulation was demonstrated. All of the inputs can be switched in under $50 \mathrm{~ns}$. Switching speed is limited by electrical connections and by the diode capacitance. The laser linewidth was characterized and compared with other lasers. Linewidth for the SGDBR laser is bias dependent.

Future work with this SGDBR laser will work toward finding specific uses for the high-speed wavelength switching. A specific OCT architecture will need to be developed, and wavelength switching speeds will need to be characterized for this architecture.

In conclusion, this thesis demonstrated the high-speed wavelength switching capabilities of SGDBR lasers. It was shown that the input segments can be switched rapidly enough for OCT measurement techniques. It was also shown that the linewidth for the laser is acceptable for OCT measurements, though it may be a limiting factor. 


\section{BIBLIOGRAPHY}

[1] Bernacil, Michael A., Shane O’Connor, Ben Maher, Andrew DeKelaita, and Dennis Derickson. "Microwave Signal Generation Using Self-heterodyning of a Fast Wavelength Switching SG-DBR Laser," IEEE International Microwave Symposium: IMS 2008, paper WE4D-05, June 2008.

[2] DeKelaita, Andrew. “Open-loop Tuning of SG-DBR Widely Tunable Lasers for Optical Coherence Tomography Applications.” Thesis. Cal Poly San Luis Obispo U, 2007.

[3] Derickson, Dennis, ed. Fiber Optic Test and Measurement. Prentice Hall, 1998. 179$193,401-420$.

[4] Derickson, Dennis, Mike Bernacil, Andrew DeKelaita, Ben Maher, Shane O'Connor, Matthew N. Sysak and Leif Johanssen. "SGDBR Monolithic Wavelength Tunable Lasers for Swept Source OCT," Biomedical Optics: BIOS 2008, SPIE Vol. 6847, January 2008.

[5] "GRINTECH GmbH 'Gradient Index Optics Technology.' " Grintech. 2008 September $<$ http://www.grintech.de/e_main_grin.htm $>$.

[6] McWilliam, Alan. "Femtosecond Cr4+:forsterite Laser for Applications in Telecommunications and Biophotonics.” 100-113. Nov. 2006. 13 May 2008 $<$ http://research-repository.st-andrews.ac.uk/bitstream/10023/261/1/Thesis.pdf $>$.

[7] "Melles Groit Optics Guide - Transformation and Magnification." Melles Groit Inc. 2008 Oct < http://www.mellesgriot.com/products/optics/gb_2_3.htm>. 
[8] NSG America, Inc. “Calculate distance.” 2005. 10 Jun 2008.

$<$ http://nsgamerica.com/index.php?page=materials_distance $>$.

[9] Simsarian, John, et al. "Less Than 5-ns Wavelength Switching With an SG-DBR Laser Less Than 5-ns Wavelength Switching With an SG-DBR Laser." IEEE Xplore 15 Feb. 2006. 12 May 2008 $<$ ieeexplore.ieee.org/iel5/68/33423/01583676.pdf?arnumber=1583676>.

[10] "Thorlabs.com - Swept Source OCT Systems." Thorlabs.com. 2008 Jun 27 $<$ http://www.thorlabs.com/NewGroupPage9.cfm?ObjectGroup_ID=2098>.

[11] Wheeler, T. L., S. D. Shackelford, and M. Koohmaraie. "Shear Force Procedures for Meat Tenderness Measurement." Agricultural Research Service, USDA 16 May 2005. 13 May 2008 $<$ arsserv0.tamu.edu/SP2UserFiles/Place/54380530/protocols/ShearForceProcedur es.pdf>.

[12] Wilkerson, Thomas D., Geary K. Schwemmer, and Bruce M. Gentry. "LIDAR Profiling of Aerosols, Clouds, and Winds by Doppler and Non-Doppler Methods.” NASA International H20 Project. 2002. 13 Nov. 2007 $<$ http://harlie.gsfc.nasa.gov/IHOP2002/Pub\&Pats/AMOS 2002 final.pdf $>$. 


\section{APPENDIX A: Frequency Modulation Data}

Front Mirror

\begin{tabular}{|r|r|}
\hline Freq (MHz) & Power (dBm) \\
\hline 10 & -10.0 \\
\hline 15 & -10.0 \\
\hline 20 & -11.0 \\
\hline 25 & -11.9 \\
\hline 30 & -12.0 \\
\hline 35 & -12.7 \\
\hline 40 & -13.0 \\
\hline 45 & -13.5 \\
\hline 50 & -14.1 \\
\hline 55 & -14.6 \\
\hline 60 & -14.9 \\
\hline 65 & -15.2 \\
\hline 70 & -15.8 \\
\hline 75 & -16.3 \\
\hline 80 & -16.7 \\
\hline 85 & -17.6 \\
\hline 90 & -18.1 \\
\hline 95 & -18.9 \\
\hline 100 & -18.6 \\
\hline
\end{tabular}

Laser biasing

Gain $=100.0 \mathrm{~mA}, \mathrm{SOA}=150 \mathrm{~mA}$

$\mathrm{BM}=0 \mathrm{~mA}$, Phase $=0 \mathrm{~mA}$

$\mathrm{FM}=2.1 \mathrm{~mA} \mathrm{DC},-70 \mathrm{dBm}$ AC $\underline{\text { Back Mirror }}$

\begin{tabular}{|r|r|}
\hline Freq (MHz) & Power (dBm) \\
\hline 10 & -7.3 \\
\hline 15 & -9.6 \\
\hline 20 & -10.3 \\
\hline 25 & -11.0 \\
\hline 30 & -12.1 \\
\hline 35 & -12.0 \\
\hline 40 & -12.5 \\
\hline 45 & -13.2 \\
\hline 50 & -13.5 \\
\hline 55 & -14.1 \\
\hline 60 & -14.2 \\
\hline 65 & -15.0 \\
\hline 70 & -15.1 \\
\hline 75 & -16.1 \\
\hline 85 & -17.0 \\
\hline 90 & -18.1 \\
\hline 95 & -18.5 \\
\hline
\end{tabular}

Laser biasing

Gain $=100.0 \mathrm{~mA}, \mathrm{SOA}=150 \mathrm{~mA}$

$\mathrm{FM}=0 \mathrm{~mA}$, Phase $=0 \mathrm{~mA}$

$\mathrm{BM}=1.3 \mathrm{~mA} \mathrm{DC},-70 \mathrm{dBm} \mathrm{AC}$ 
Phase

\begin{tabular}{|r|r|}
\hline Freq (MHz) & Power (dBm) \\
\hline 10 & -17.4 \\
\hline 15 & -18.3 \\
\hline 20 & -18.8 \\
\hline 25 & -19.0 \\
\hline 30 & -19.9 \\
\hline 35 & -19.1 \\
\hline 40 & -19.4 \\
\hline 45 & -20.4 \\
\hline 50 & -21.4 \\
\hline 55 & -21.7 \\
\hline 60 & -21.1 \\
\hline 65 & -22.9 \\
\hline 70 & -22.5 \\
\hline 75 & -24.5 \\
\hline 80 & -25.0 \\
\hline 85 & -27.4 \\
\hline 90 & -26.7 \\
\hline 95 & -27.9 \\
\hline 100 & -29.2 \\
\hline 200 & -34.7 \\
\hline 300 & -38.9 \\
\hline 400 & -42.8 \\
\hline 500 & -46.4 \\
\hline
\end{tabular}

Laser biasing

Gain $=100.0 \mathrm{~mA}, \mathrm{SOA}=150 \mathrm{~mA}$ $\mathrm{BM}=0 \mathrm{~mA}, \mathrm{FM}=0 \mathrm{~mA}$

Phase $=3.4 \mathrm{~mA} \mathrm{DC},-70 \mathrm{dBm}$ AC 
APPENDIX B: LabView Program for Collecting Data from ESA

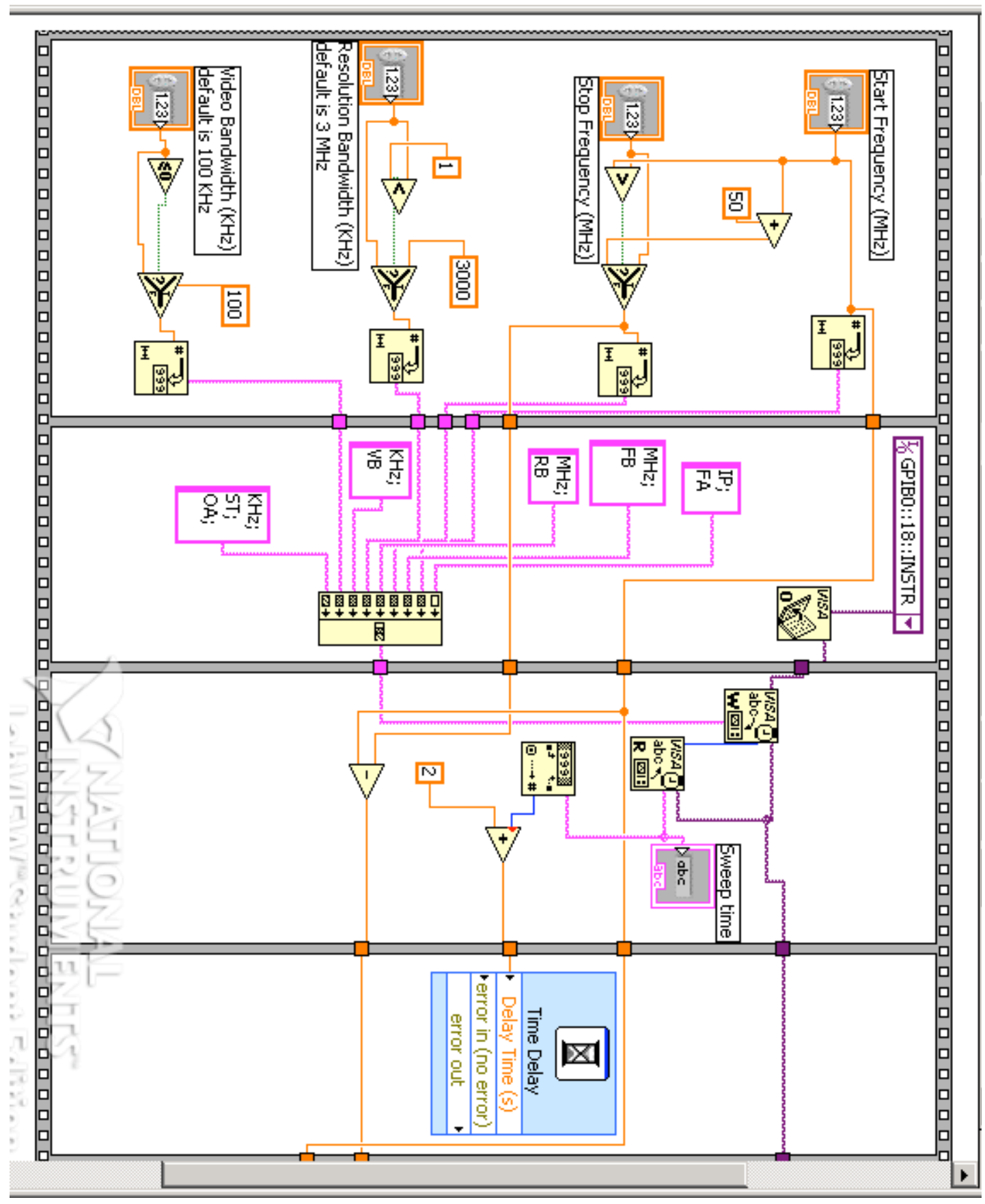




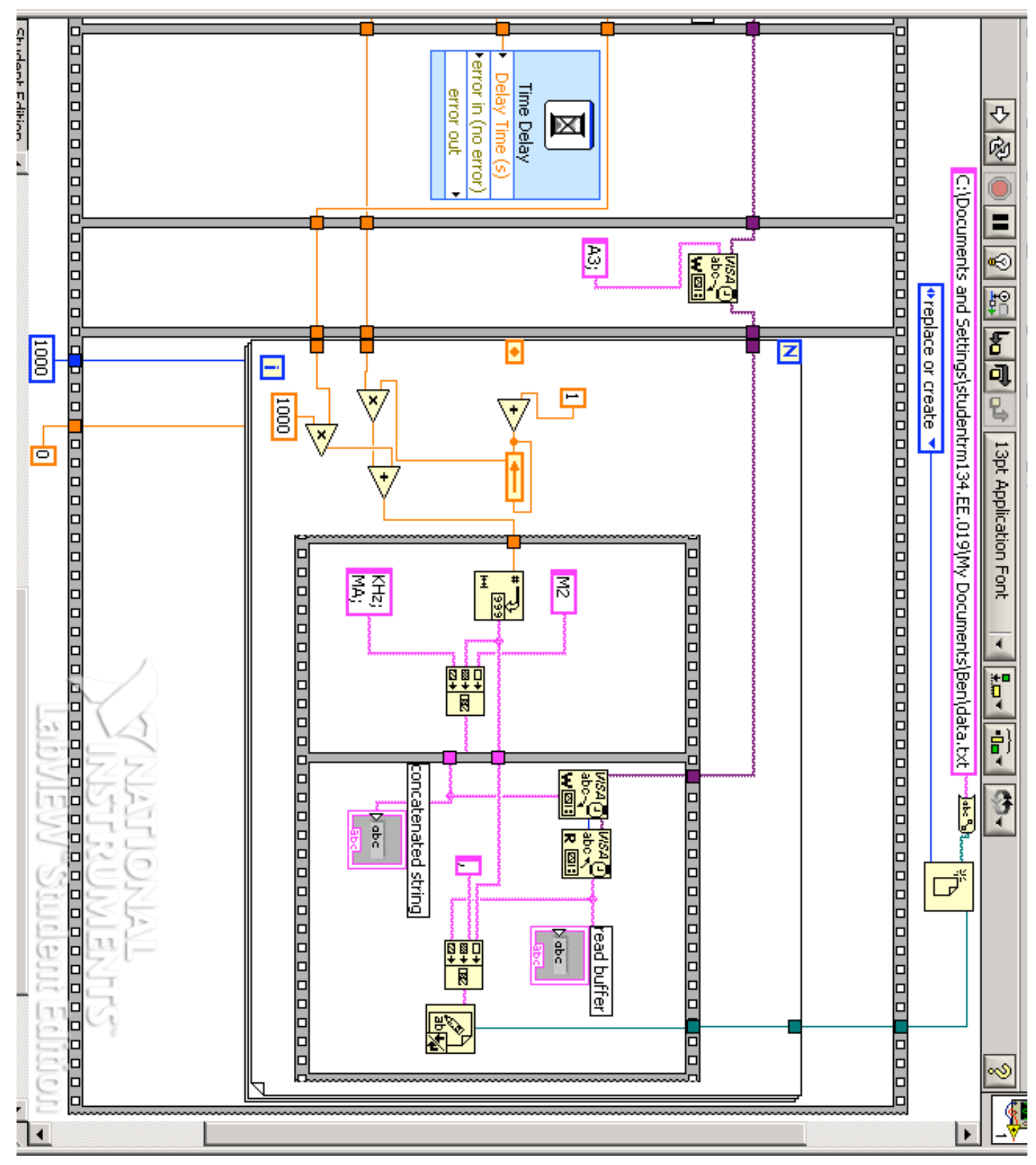




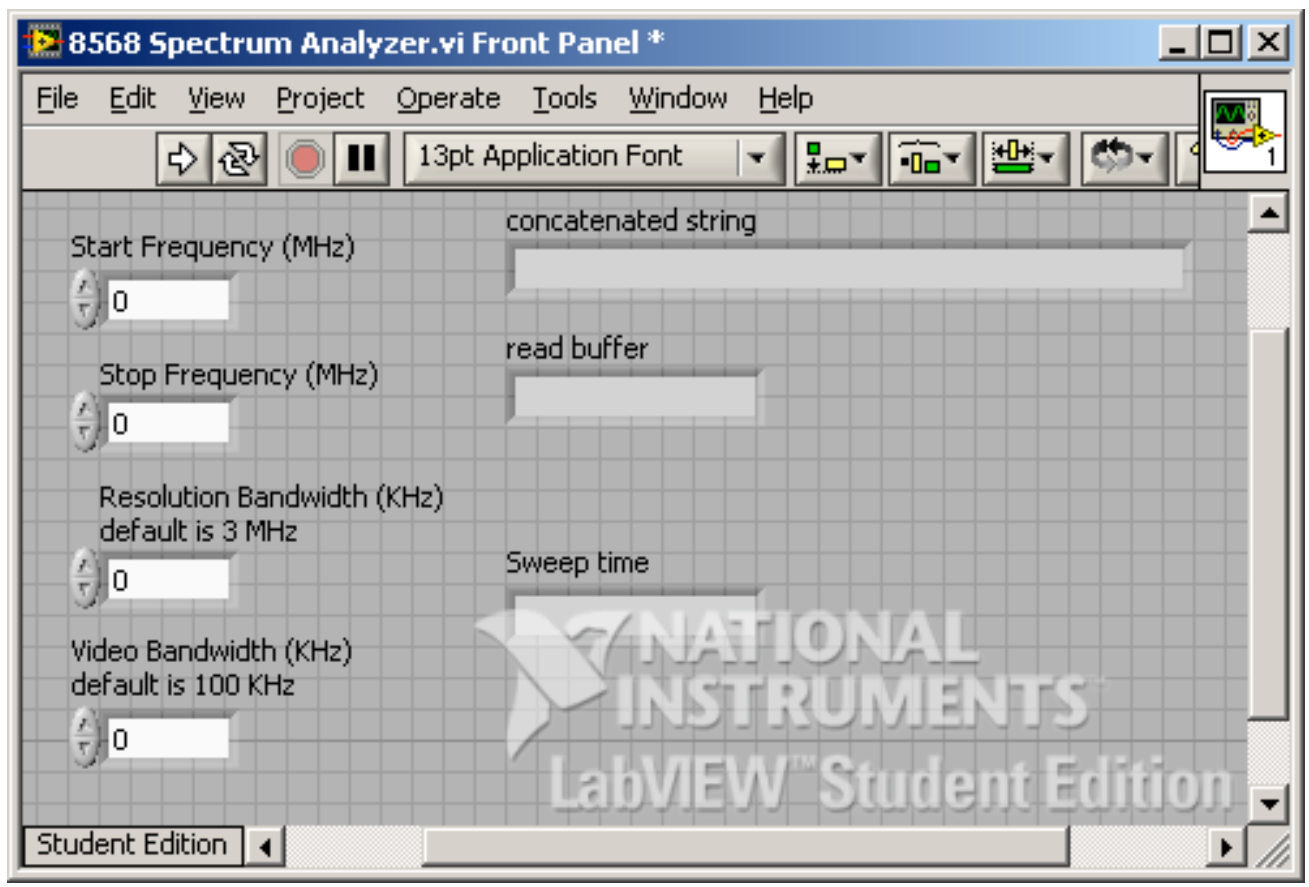


APPENDIX C: Wavelength vs. Phase Current for Chip Laser

\begin{tabular}{|r|c|r|}
\hline Phase current $\mathbf{( m A )}$ & $\boldsymbol{\lambda} \mathbf{( n m})$ & Peak Power $\mathbf{( d B m )}$ \\
\hline 2.0 & 1566.25 & -74.3 \\
\hline 2.5 & 1566.22 & -71.7 \\
\hline 3.0 & 1566.20 & -70.2 \\
\hline 3.5 & 1566.18 & -69.9 \\
\hline 4.0 & 1566.16 & -69.8 \\
\hline 4.5 & 1566.14 & -70.0 \\
\hline 5.0 & 1566.13 & -70.2 \\
\hline
\end{tabular}


APPENDIX D: Pin Layout of the Packaged SGDBR laser

\section{Pin Descriptions}

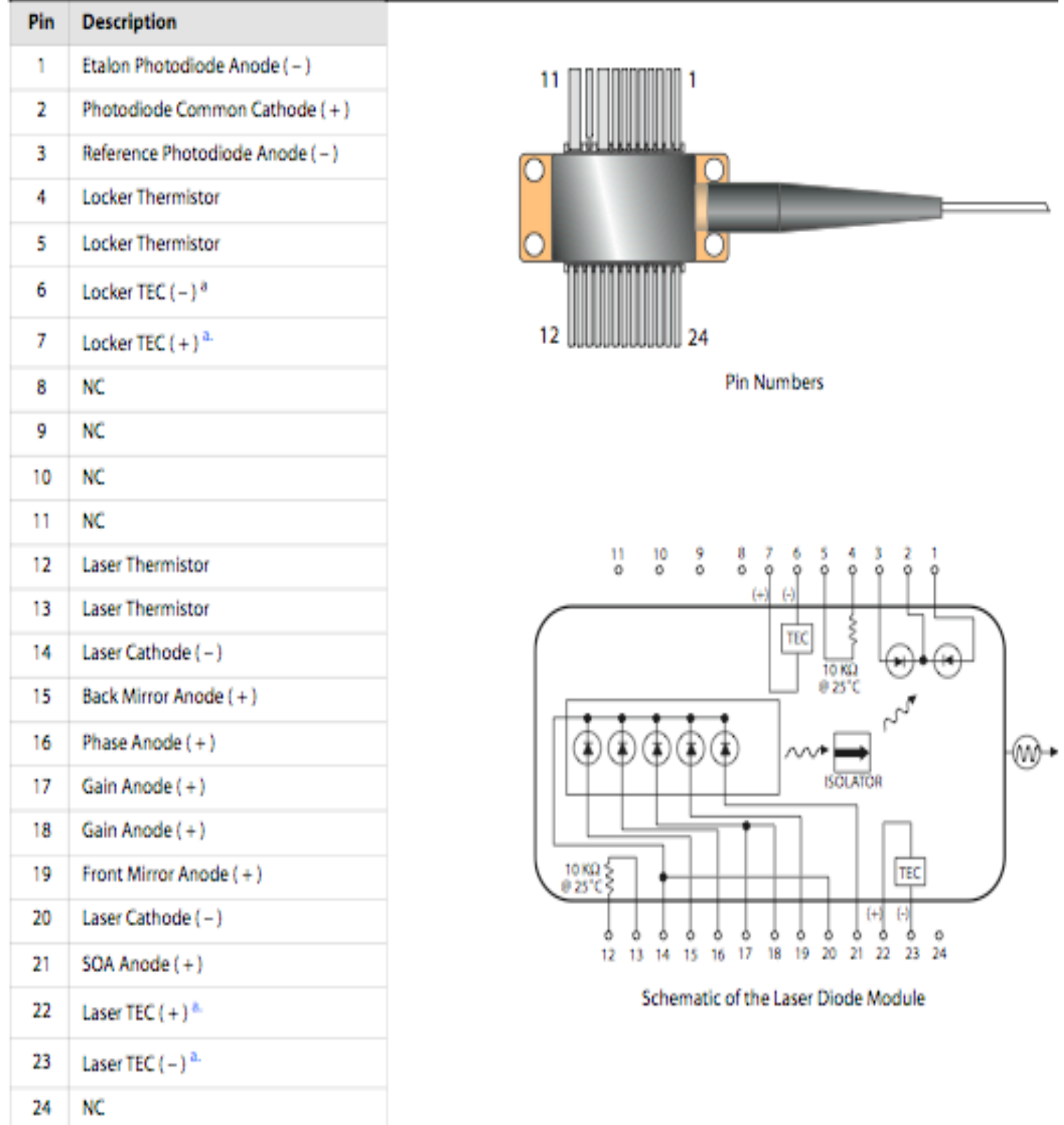

a. Positive current through the laser of locker TEC cools the laser or locker. 
APPENDIX E: Amplitude Modulation Data

Gain

\begin{tabular}{|r|r|}
\hline $\begin{array}{r}\text { Frequency } \\
\text { (MHz) }\end{array}$ & $\begin{array}{c}\text { Power } \\
\text { (dBm) }\end{array}$ \\
\hline 10 & -1.7 \\
\hline 20 & -0.5 \\
\hline 30 & -2.7 \\
\hline 40 & -1.7 \\
\hline 50 & -1.7 \\
\hline 60 & -1.9 \\
\hline 70 & -1.7 \\
\hline 80 & -1.2 \\
\hline 90 & -1.7 \\
\hline 100 & -2.0 \\
\hline 150 & -1.8 \\
\hline 200 & -3.7 \\
\hline 250 & -3.7 \\
\hline 300 & -3.2 \\
\hline 350 & -5.2 \\
\hline 400 & -6.8 \\
\hline 450 & -6.1 \\
\hline 500 & -6.5 \\
\hline 550 & -8.4 \\
\hline 600 & -9.6 \\
\hline 650 & -10.2 \\
\hline 700 & -9.1 \\
\hline 750 & -12.3 \\
\hline 800 & -12.2 \\
\hline
\end{tabular}

$\underline{\text { SOA }}$

\begin{tabular}{|c|c|}
\hline $\begin{array}{c}\text { Frequency } \\
\text { (MHz) }\end{array}$ & $\begin{array}{l}\text { Power } \\
(\mathrm{dBm})\end{array}$ \\
\hline 10 & -1.0 \\
\hline 20 & -0.1 \\
\hline 30 & -0.5 \\
\hline 40 & -0.2 \\
\hline 50 & -0.4 \\
\hline 60 & -0.6 \\
\hline 70 & -1.0 \\
\hline 80 & -0.8 \\
\hline 90 & -1.2 \\
\hline 100 & -1.7 \\
\hline 150 & -1.5 \\
\hline 200 & -2.8 \\
\hline 250 & -4.5 \\
\hline 300 & -3.8 \\
\hline 350 & -5.2 \\
\hline 400 & -3.0 \\
\hline 450 & -6.7 \\
\hline 500 & -7.5 \\
\hline 550 & -9.4 \\
\hline 600 & -11.4 \\
\hline 650 & -12.6 \\
\hline 700 & -9.3 \\
\hline 750 & -12.3 \\
\hline 800 & -15.3 \\
\hline
\end{tabular}


Phase

\begin{tabular}{|r|r|}
\hline \multicolumn{1}{|c|}{$\begin{array}{c}\text { Frequency } \\
\text { (MHz) }\end{array}$} & $\begin{array}{c}\text { Power } \\
\text { (dBm) }\end{array}$ \\
\hline 10 & -27.4 \\
\hline 15 & -28.7 \\
\hline 20 & -28.4 \\
\hline 25 & -28.9 \\
\hline 30 & -28.9 \\
\hline 35 & -30.0 \\
\hline 40 & -29.6 \\
\hline 45 & -29.9 \\
\hline 50 & -30.6 \\
\hline 55 & -30.7 \\
\hline 60 & -32.0 \\
\hline 65 & -31.1 \\
\hline 70 & -32.2 \\
\hline 75 & -32.8 \\
\hline 80 & -32.7 \\
\hline 85 & -34.3 \\
\hline 90 & -35.0 \\
\hline 95 & -36.0 \\
\hline 100 & -36.1 \\
\hline 105 & -37.6 \\
\hline 110 & -38.9 \\
\hline 115 & -38.7 \\
\hline 120 & -39.9 \\
\hline & \\
\hline 105
\end{tabular}

Front Mirror

\begin{tabular}{|r|r|}
\hline \multicolumn{1}{|c|}{$\begin{array}{c}\text { Frequency } \\
\text { (MHz) }\end{array}$} & $\begin{array}{c}\text { Power } \\
\text { (dBm) }\end{array}$ \\
\hline 10 & -26.8 \\
\hline 15 & -24.7 \\
\hline 20 & -24.8 \\
\hline 25 & -25.6 \\
\hline 30 & -26.3 \\
\hline 35 & -27.3 \\
\hline 40 & -27.4 \\
\hline 45 & -27.9 \\
\hline 50 & -28.8 \\
\hline 55 & -29.8 \\
\hline 60 & -29.8 \\
\hline 65 & -30.1 \\
\hline 70 & -31.7 \\
\hline 75 & -32.3 \\
\hline 80 & -33.3 \\
\hline 85 & -33.6 \\
\hline 90 & -34.2 \\
\hline 95 & -35.2 \\
\hline 100 & -36.1 \\
\hline 105 & -39.0 \\
\hline 110 & -40.8 \\
\hline 115 & -40.5 \\
\hline 120 & -40.0 \\
\hline & \\
\hline
\end{tabular}


$\underline{\text { Back Mirror }}$

\begin{tabular}{|c|c|}
\hline $\begin{array}{l}\text { Frequency } \\
(\mathrm{MHz})\end{array}$ & $\begin{array}{l}\text { Power } \\
\text { (dBm) }\end{array}$ \\
\hline 10 & -25.4 \\
\hline 15 & -26.8 \\
\hline 20 & -29.1 \\
\hline 25 & -29.8 \\
\hline 30 & -28.8 \\
\hline 35 & -29.1 \\
\hline 40 & -29.5 \\
\hline 45 & -31.5 \\
\hline 50 & -33.0 \\
\hline 55 & -36.2 \\
\hline 60 & -35.3 \\
\hline 65 & -35.3 \\
\hline 70 & -36.7 \\
\hline 75 & -37.8 \\
\hline 80 & -38.8 \\
\hline 90 & -39.0 \\
\hline 95 & -40.7 \\
\hline 100 & -42.2 \\
\hline 105 & -43.5 \\
\hline 110 & -42.7 \\
\hline 115 & -44.8 \\
\hline 120 & -44.6 \\
\hline 125 & -44.4 \\
\hline
\end{tabular}

